\title{
Tempo de chegada ao equilíbrio da dinâmica de Metropolis para o GREM
}

\author{
Antonio Marcos Batista do Nascimento
}

\section{TESE APRESENTADA}

$\mathrm{AO}$

\author{
Instituto De MatemáticA E EstatísticA \\ DA \\ Universidade de SÃo Paulo \\ PARA \\ OBTENÇÃO DO TÍTULO \\ $\mathrm{DE}$ \\ DOUTOR EM CIÊNCIAS
}

Programa: Estatística

Orientador: Prof. Dr. Luiz Renato Gonçalves Fontes

Durante o desenvolvimento deste trabalho o autor recebeu auxílio financeiro da CAPES e

$\mathrm{CNPq}$

São Paulo, maio de 2018 


\section{Tempo de chegada ao equilíbrio da dinâmica de Metropolis para o GREM}

Esta é a versão original da tese elaborada pelo

candidato Antonio Marcos Batista do Nascimento, tal como submetida à Comissão Julgadora. 


\title{
Tempo de convergência ao equílibrio da dinâmica de Metropolis para o GREM
}

\author{
Esta versão da tese contém as correções e alterações sugeridas \\ pela Comissão Julgadora durante a defesa da versão original do trabalho, \\ realizada em 29/03/2018. Uma cópia da versão original está disponível no \\ Instituto de Matemática e Estatística da Universidade de São Paulo.
}

Comissão Julgadora:

- Prof. Dr. Luiz Renato Gonçalves Fontes (orientador) - IME-USP

- Prof. Dr. Jean-Georges Pierre Picco - Aix-Marseille

- Prof ${ }^{a}$. Dra ${ }^{a}$ Maria Eulália Vares - UFRJ

- Prof. Dr. Rodrigo Bissacot Proença - IME-USP

- Prof. Dr. Anatoli Lambartsev - IME-USP 


\section{Agradecimentos}

Primeiramente, gostaria de agradecer a minha família pelo suporte, apoio e motivação manisfestados ao longo de todos esses anos de doutorado, sempre me estimulando a buscar realizar meus sonhos e objetivos. Saibam que me orgulho e amo muito todos vocês.

Gostaria também de agradecer ao meu orientador, Prof. Luiz Renato, por todos os ensinamentos, pela sua enorme paciência em ensinar, pela experiência repassada, pelas estimulantes discussões, ajuda nos momentos difíceis e, principalmente, por seus comentários e sugestões a respeito da tese, as quais, em sua maioria, com a finalidade de torná-la mais simples para o leitor. Serei para sempre grato ao senhor.

Gostaria ainda de agradecer, de um modo geral, a todos os meus amigos e colegas com quem compartilhei experiências, opiniões e ideias. Definitivamente, vocês tornaram essa jornada bem mais simples e divertida.

Por fim, gostaria de agradecer a todo o Departamento de Estatística da Universidade de São Paulo, a CAPES e ao CNPq pelo apoio financeiro e aos professores que compuseram a banca de defesa de minha tese de doutorado. 


\section{Resumo}

\section{NASCIMEnTO, A. M. B. Tempo de chegada ao equilíbrio da dinâmica de Metropolis}

para o GREM. 2018. Tese (Doutorado) - Instituto de Matemática e Estatística, Universidade de São Paulo, São Paulo, 2018.

Neste trabalho consideramos um processo de Markov a tempo contínuo com espaço de estados finito em um meio aleatório, a saber, a dinâmica de Metropolis para o Modelo de Energia Aleatória Generalizado (GREM) com um número de níveis finito e discutimos o comportamento do seu tempo de chegada ao equilíbrio, o qual é dado pelo inverso da lacuna espectral de sua matriz de probabilidades de transição. No principal resultado desta tese provamos que o quociente entre o volume do sistema e o logaritmo do inverso da lacuna é quase sempre limitado, por cima, por uma função da temperatura, que também é a que descreve a energia livre do GREM sob o regime de temperaturas baixas. Como um estudo adicional, também é discutido um correspondente limitante inferior em um caso particular do GREM com 2 níveis.

Palavras-chave: GREM, vidros de spins, dinâmica de Metropolis, lacuna espectral, convergência ao equilíbrio, desigualdade de Poincaré. 


\section{Abstract}

NASCIMENTO, A. M. B. Reaching time to equilibrium of the Metropolis dynamics for the GREM. 2018. Tese (Doutorado) - Instituto de Matemática e Estatística, Universidade de São Paulo, São Paulo, 2018.

In this work we consider a finite state continuous-time Markov process in a random environment, namely, the Metropolis dynamics for the Generalized Random Energy Model (GREM) with a finite number of levels, and we discuss the behavior of its reaching time to equilibrium which is given by inverse of the spectral gap of its transition probability matrix. On the main result of this thesis, we prove the division between the system volume and the logarithm of the inverse of the gap is almost surely upper bounded by a function of the temperature that it is also the function that describe the free energy of the GREM at low temperature. As an additional study, it is also discuss the corresponding limiting lower in a particular case of the 2-level GREM.

Keywords: GREM, spin glasses, Metropolis dynamics, spectral gap, convergence to equilibrium, Poincaré inequality. 


\section{Conteúdo}

Conteúdo vii

$\begin{array}{ll}\text { Nomenclatura } & \text { ix }\end{array}$

1 Introdução 1

2 A dinâmica de Metropolis no GREM 5

2.1 O Modelo . . . . . . . . . . . . . . . . . . . . . . 6

2.2 A dinâmica . . . . . . . . . . . . . . . . . . . . . . . . . . 14

2.3 Resultado Principal . . . . . . . . . . . . . . . . . . . . . 16

3 Estratégia Geral $\quad 19$

3.1 A Desigualdade de Poincaré . . . . . . . . . . . . . . . . . . 20

3.2 O Conjunto de Caminhos . . . . . . . . . . . . . . . . . . 21

3.2.1 Motivações e Escolha do Conjunto de Caminhos . . . . . . . . . . . . . . 21

3.2 .2 Primeiras Estimativas . . . . . . . . . . . . . . . . . 32

4 Estimativa Principal $\quad 35$

4.1 Estimativas Preparatórias _ . . . . . . . . . . . . . . . . . 35

4.2 Estimativa Intermediária . . . . . . . . . . . . . . . . . . . . . . . 41

4.2 .1 Introdução . . . . . . . . . . . . . . . . . . . . . . . . . . . . 41

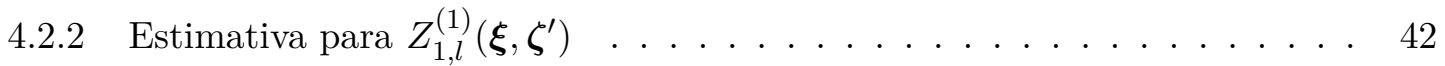

4.2 .3 Estimativa de Grandes Desvios . . . . . . . . . . . . . . . . . . . . 44

$4.2 .4 \quad$ Estimativa Determinística . . . . . . . . . . . . . . . . . . . . 49 
4.2.5 Conclusão da Estimativa para $(4.1 .34) \ldots \ldots \ldots \ldots$. . . . . . . . 54

4.2 .6 Prova da Proposição $4.2 .2 \ldots \ldots \ldots$. . . . . . . . . . . . . . 57

4.3 Conclusão . . . . . . . . . . . . . . . . . . . . . . . . . 63

5 Conclusão da Prova do Teorema 2.3.1 $\quad 65$

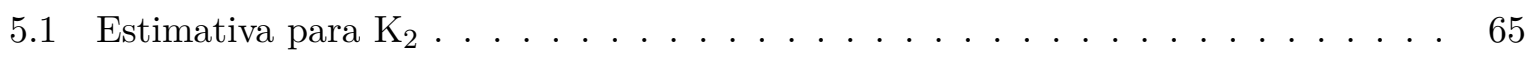

5.2 Conclusão da Prova do Teorema $2.3 .1 \ldots \ldots$. . . . . . . . . . . 70

6 Cota Inferior para o Inverso da Lacuna: um caso particular $\quad 73$

6.1 Introdução e Resultados . . . . . . . . . . . . . . . . . . . . . . . 73

6.2 Demonstração do Teorema $6.1 .1 \ldots \ldots \ldots \ldots$. . . . . . . . . 76

6.3 Demonstração do Teorema $6.1 .2 \ldots \ldots \ldots \ldots \ldots$. . . . . . . . . 78

$\begin{array}{ll}\text { A Resultados de Matemática Geral } & 83\end{array}$

B Resultados de Teoria de Probabilidade $\quad 85$

$\begin{array}{lr}\text { Bibliografia } & 87\end{array}$ 


\section{Nomenclatura}

$(\mathscr{S}, \Sigma, \mathbb{P})$ espaço de probabilidade consistindo de um conjunto não vazio, uma $\sigma$-álgebra e uma medida de probabilidade

$\mathbb{1} \quad$ função indicadora/característica

$\beta \quad$ o inverso da temperatura

d distância induzida pelo produto interno usual em $\mathbb{R}^{n}$

$\mathrm{d}_{1} \quad$ distância de Hamming em $\Omega_{\mathcal{V}_{1}(1)}$

$\mathcal{D}_{\mathcal{V}}\left(\boldsymbol{\eta}, \boldsymbol{\eta}^{\prime}\right)$ conjunto de discrepâncias entre as configurações $\boldsymbol{\eta}$ e $\boldsymbol{\eta}^{\prime}$

$\mathrm{d}_{\mathrm{H}} \quad$ distância de Hamming

$\mathbb{E} \quad$ esperança

$\mathcal{E} \quad$ conjunto de elos de $\Omega_{\mathcal{V}}$

$\mathscr{E}(\phi, \phi)$ forma de Dirichlet associado ao semigrupo de Markov $\mathrm{P}_{t}$

$\gamma \quad$ caminho sobre o hipercubo $\Omega \mathcal{v}$

$\gamma_{\boldsymbol{\eta}, \boldsymbol{\eta}^{\prime}}$ caminho sobre $\Omega_{\mathcal{V}}$ indo de $\boldsymbol{\eta}$ para $\boldsymbol{\eta}^{\prime}$

$\mathcal{I}_{k} \quad$ denota o conjunto $\{1, \ldots, k\}$

$\langle\cdot, \cdot\rangle \quad$ o produto interno usual em $\mathbb{R}^{n}$

$\mathscr{L} \quad$ gerador de Markov

$\mu \quad$ medida de probabilidade(Gibbs)

$\Omega_{\mathcal{V}}, \Omega_{\mathcal{V}_{j}}$ o espaço de configurações do sistema, espaço de configurações do nível $j$

P matriz de probabilidades de transição tipo-Metropolis

$\mathrm{P}_{t} \quad$ semigrupo markoviano a tempo contínuo 
$\boldsymbol{\sigma}, \sigma_{j}$ denota um elemento de $\Omega \mathcal{V}$, denota um elemento de $\Omega_{\mathcal{V}_{j}}$

$\tau \quad$ inverso da lacuna espectral de $\mathscr{L}$ (ou da matriz $\mathrm{P}$ )

$\overline{\boldsymbol{\sigma}} \quad$ (única) configuração de $\Omega_{\mathcal{V}}$ tal que $H(\overline{\boldsymbol{\sigma}})=\min _{\boldsymbol{\sigma} \in \Omega_{\mathcal{V}}} H(\boldsymbol{\sigma})$

$\mathcal{V}, \mathcal{V}_{j}$ o conjunto de partículas do sistema, o conjunto de partículas do nível $j$

$\operatorname{Var}_{\mu} \quad$ variância com respeito a medida de probabilidade $\mu$

$\mathscr{X} \quad$ é a família de gaussianas padrão independentes sobre $(\mathscr{S}, \Sigma, \mathbb{P})$

$\left\{\beta_{\ell}\right\}_{\ell=1}^{\ell_{n}}$ sequência de temperaturas inversas críticas

$F_{n}(\beta)$ função energia livre

$F_{N, n}(\beta)$ energia livre à volume finito

$H \quad$ Hamiltoniano

$N, n$ inteiro positivo que eventualmente diverge, o número de níveis da hierarquia

$V, V_{j} \quad$ o volume de partículas do sistema, o volume de partículas do nível $j$

Z $\quad$ função de partição à volume finito 


\section{Lista de Figuras}

2.1 Exemplo de uma estrutura de árvore de um GREM com 3 níveis. Neste caso, estamos considerando que $V_{1}=2$ e $V_{2}=V_{3}=1 \ldots \ldots \ldots \ldots$

4.1 Seja $\Omega_{\mathcal{V}}$ o hipercubo obtido fazendo $\mathrm{B}=n=3$ e $\mathrm{v}_{1}=\mathrm{v}_{2}=\mathrm{v}_{3}$. A figura (a) ilustra os possíveis vértices $\boldsymbol{\eta}$ e $\boldsymbol{\eta}^{\prime}$ em $\Omega_{\mathcal{V}}$ tais que o caminho $\gamma_{\boldsymbol{\eta}, \boldsymbol{\eta}^{\prime}}^{1}$ passa por meio do elo $e=\left(\boldsymbol{\sigma}, \boldsymbol{\sigma}^{\prime}\right)$. O spin de discordância entre $\boldsymbol{\sigma}$ e $\boldsymbol{\sigma}^{\prime}$ está representado por $\circ$ e $\bullet$, respectivamente. Em $\boldsymbol{\eta}$, respectivamente em $\boldsymbol{\eta}^{\prime}$, a linha em zigue-zague representa os spins possivelmente trocados na construção do caminho $\gamma_{\boldsymbol{\eta}, \boldsymbol{\eta}^{\prime}}^{1}$ antes de $\boldsymbol{\sigma}$, respectivamente depois de $\boldsymbol{\sigma}^{\prime}$. As figuras (b) e (c) ilustram o mesmo que (a), mas, agora, para o elo $e^{\prime}=\left(\hat{\boldsymbol{\sigma}}, \hat{\boldsymbol{\sigma}}^{\prime}\right) e^{\prime \prime}=\left(\tilde{\boldsymbol{\sigma}}, \tilde{\boldsymbol{\sigma}}^{\prime}\right)$, respectivamente. . . . . .

5.1 A figura acima ilustra um caminho $\gamma_{\boldsymbol{\eta}, \boldsymbol{\eta}^{\prime}}=\gamma_{\boldsymbol{\eta}, \boldsymbol{\omega}}^{1} \cup \gamma_{\boldsymbol{\omega}, \boldsymbol{\eta}^{\prime}} \operatorname{com} \gamma_{\boldsymbol{\eta}, \boldsymbol{\omega}}^{1} \ni e=\left(\boldsymbol{\sigma}, \boldsymbol{\sigma}^{\prime}\right)$. Em $\boldsymbol{\eta}$, respectivamente em $\boldsymbol{\omega}$, a linha em zigue-zague representa os spins possivelmente trocados na construção do caminho $\gamma_{\boldsymbol{\eta}, \boldsymbol{\omega}}^{1}$ antes de $\boldsymbol{\sigma}$, respectivamente depois de $\boldsymbol{\sigma}^{\prime}$. Chamamos atenção ao fato que o caminho $\gamma_{\boldsymbol{\omega}, \boldsymbol{\eta}^{\prime}}$ é obtido trocando-se apenas os spins das coordenadas pertencentes ao conjunto $\mathcal{D}_{\mathcal{V}_{1}(1)}\left(\boldsymbol{\omega}, \boldsymbol{\eta}^{\prime}\right)$ que estão representadas pelas bolas cheias no primeiro bloco de $\boldsymbol{\eta}^{\prime} \ldots \ldots \ldots$. . . . . . 67 


\section{Capítulo 1}

\section{Introdução}

Nestas últimas décadas vidros de spins tem se tornado uma fascinante e complexa questão da Teoria de Probabilidade, não só pela complexidade dos problemas "estáticos", mas também pela variedade dos fenômenos "dinâmicos" que tem surgido.

Do ponto de vista teórico vidros de spins são sistemas de spins interagindo aleatoriamente em uma rede. E como é bem conhecido, uma descrição de campo médio de tais sistemas é dado pelo modelo de Sherrington e Kirkpatrick [SK75], hoje conhecido simplesmente como modelo SK. Este modelo é definido sobre um rede unidimensional de volume $N$ com a energia total de cada configuração $\boldsymbol{\sigma}=\left\{\sigma_{i}\right\}_{i=1}^{N}$ do sistema dada pela soma

$$
H(\boldsymbol{\sigma})=-\sum_{1 \leq i<j \leq N} \frac{J_{i j}}{\sqrt{N}} \sigma_{i} \sigma_{j}
$$

em que $\sigma_{i}= \pm 1$ e $J_{i j}$ são variáveis aleatórias gaussianas padrão independentes. Apesar da aparente simplicidade, o estudo desse modelo tem provado ser matematicamente não-trivial, até mesmo de forma heurística, devido à rica e complicada estrutura de correlações entre as energias de seu sistema.

O Modelo de Energia Aleatória (REM) foi introduzido por Derrida [Der80] como uma simplificação do modelo SK. Este modelo, também definido em um volume $N$, consiste em considerar as energias das configurações do sistema como variáveis aleatórias gaussianas independentes de média zero e variância $N$. Portanto, sua função de partição é dada pela seguinte expressão:

$$
Z_{N, 1}(\beta)=\sum_{i=1}^{2^{N}} e^{-\beta \sqrt{N} X_{i}}, \quad X_{i} \sim \mathcal{N}(0,1)
$$

em que $\beta>0$ é o parâmetro inverso da temperatura. Isto é considerado pela comunidade física uma simplificação extrema para vidros de spins, já que desconsidera qualquer estrutura de dependência entre as energias do sistema, algo presente em modelos mais realistas como, por exemplo, o SK. 
Com a proposta de incorporar alguma estrutura de dependência entre as energias do sistema e assim obter um modelo mais realista para vidros de spins, Derrida [Der85] propôs então uma generalização do REM. Ele escolheu uma estrutura hierárquica em níveis para definir variáveis aleatórias gaussianas fortemente correlacionadas e o chamou de Modelo de Energia Aleatória Generalizado (GREM). Assim como REM, as $2^{N}$ energias do sistema não são especificadas, mas a energia de cada configuração é a soma de variáveis aleatórias gaussianas independentes. Uma descrição do GREM segue. Sejam $n, N \in \mathbb{N}$ tais que $n \leq N$ e sejam $\left\{a_{j}\right\}_{j=1}^{n}$ e $\left\{\alpha_{j}\right\}_{j=1}^{n}$ duas sequências finitas de números reais, tais que $a_{j}>0$ e $\alpha_{j}>1$ para todo $j=1, \ldots, n$, e

$$
\sum_{j=1}^{n} a_{j}=1 \quad \text { e } \quad \prod_{j=1}^{n} \alpha_{j}=2 .
$$

Seja $(\mathscr{S}, \Sigma, \mathbb{P})$ um espaço de probabilidade, tal que para todo $n \in \mathbb{N}$ (fixo) e todo $N \in \mathbb{N}$ existe uma família de $\alpha_{1}^{N}+\left(\alpha_{1} \alpha_{2}\right)^{N}+\cdots+\left(\alpha_{1} \cdots \alpha_{n}\right)^{N}$ variáveis aleatórias gaussianas padrão independentes, digamos

$$
X_{i_{1}, \ldots, i_{j}}^{j} \sim \mathcal{N}(0,1), \quad j=1, \ldots, n \text { e } i_{j}=1, \ldots, \alpha_{j}^{N}
$$

O GREM ao inverso de temperatura $\beta>0$ é então definido pela função de partição,

$$
Z_{N, n}(\beta)=\sum_{i_{1}=1}^{\alpha_{1}^{N}} \sum_{i_{2}=1}^{\alpha_{2}^{N}} \cdots \sum_{i_{n}=1}^{\alpha_{n}^{N}} e^{-\beta \sqrt{N} \sum_{j=1}^{n} \sqrt{a_{j}} X_{i_{1}, \ldots, i_{j}}^{j}} .
$$

Não é difícil ver que o GREM corresponde ao REM quando $n=1$.

Objetivos da Tese. Uma das características de vidros de spins é que eles são sistemas que convergem tão lentamente para o equilíbrio sob o regime de temperaturas baixas que nunca são observados próximo do equilíbrio em simulações ou em experimentos em laboratórios (veja [BCKM98]). Dessa característica surge então o seguinte questionamento:

\section{Quanto tempo é necessário esperar para observar o sistema próximo de seu equilíbrio?}

A proposta desta tese é então considerar uma versão dinâmica do GREM, a saber, o GREM submetido a dinâmica de Metropolis (ou seja, um processo de Markov a tempo contínuo reversível com respeito à medida de equilíbrio), e discutir o comportamento do tempo de convergência ao equilíbrio (em função da temperatura) desta dinâmica. Nosso principal objetivo é obter uma estimativa para o tempo de convergência ou, equivalentemente, para a lacuna espectral da dinâmica, isto é, a diferença entre o primeiro e o segundo autovalor da matriz de transição do correspondente processo de Markov a tempo contínuo. Um objetivo secundário é obter alguma 
informação sobre o comportamento exato da lacuna da dinâmica. Isto será feito em um caso particular do modelo.

O GREM é um dos poucos modelos de vidros de spins em que uma análise matemática rigorosa tem provado ser possível não só em questões estáticas (por exemplo, Capocaccia et al. [CCP87], Dorlas e Dukes [DD02], Bovier e Kurkova [BK04a, BK04b] e Jana e Rao [JR06]), como também em questões dinâmicas (por exemplo, Arous et al. [ABG01, ABG02, ABG03a, ABG03b], Gayrard [Gay16a, Gay16b] e Černỳ e Wassmer [ČW17]). Vale destacar que a maioria dos avanços rigorosos em problemas dinâmicos, se não todos, têm sido com análise no REM. Para casos mais gerais do GREM, a maioria dos problemas dinâmicos ainda continuam sem resposta.

Os trabalhos de Fontes et al. [FIKP98], de Mathieu [Mat00] e de Mathieu e Picco [MP] representam os únicos artigos com resultados sobre tempos de convergência ao equilíbrio de dinâmicas para o GREM, mas todos eles com a análise restrita ao REM. De particular interesse são os resultados de Fontes et al. que descreveram o comportamento exato da lacuna da dinâmica de Metropolis para o REM. Estenderemos nesta tese um dos resultados de [FIKP98] para o GREM.

Contribuições e Organização da Tese: Vamos agora descrever em mais detalhes o que fizemos em nosso trabalho destacando suas contribuições. Apresentamos no Capítulo 2 o processo markoviano a tempo contínuo, o GREM sob a dinâmica de Metropolis, que será o objeto de nosso estudo e o resultado principal desta tese, o Teorema 2.3.1.

Em [FIKP98], em que o REM foi estudado também sob Metropolis, o principal resultado é a descrição completa e exata do comportamento assintótico da lacuna espectral (Teorema 1). De fato, Fontes et al. provaram que, denotando-se por $\lambda_{N}$ a lacuna espectral da dinâmica, para todo $\beta>0$,

$$
\lim _{N \uparrow \infty} \frac{1}{N} \log \frac{1}{\lambda_{N}}=\beta \sqrt{2 \log 2}
$$

quase certamente. Cabe destacar que a expressão do lado direito de (1.0.6), vista como função de $\beta$, é também a função que descreve a energia livre do REM a baixas temperaturas. Infelizmente, não seremos capazes de provar uma afirmação análoga para o caso do GREM. Nesta tese, provaremos que para a dinâmica de Metropolis no GREM, para todo $\beta>0$,

$$
\limsup _{N \uparrow \infty} \frac{1}{N} \log \frac{1}{\lambda_{N}}
$$

é quase sempre limitado superiormente pela mesma expressão que descreve a energia livre do GREM sob baixas temperaturas (Teorema 2.3.1). Essa afirmação generaliza a Proposição 4.2 em [FIKP98]. Obtivemos essa proposição como corolário do nosso resultado. Além disso, se o 
interesse é estimar a distância (em variação total) entre a lei do processo de Markov no tempo $t$, iniciando em um dado estado, e a sua medida de equilíbrio, então é somente de uma cota superior para o inverso da lacuna que se faz necessário (cf. (2.3.6)).

Para provar o Teorema 2.3.1 usaremos uma desigualdade tipo Poincaré derivada por Sinclair em [Sin91] (cf. também a desigualdade de Poincaré derivada em [DS91]), a qual é dada em termos de um conjunto de caminhos que construiremos cuidadosamente no Capítulo 3 explorando o fato que o espaço de estados da cadeia de Markov em estudo pode ser dividido em elementos "bons" e "ruins". No Capítulo 4 apresentaremos a estimativa do termo que de fato contribui para o tamanho da lacuna da dinâmica, o que nos leva ao Capítulo 5, em que reunimos todas as estimativas em um argumento para concluir a demonstração do teorema principal.

O Capítulo 6 tem como objetivo obter alguma informação sobre o comportamento exato da lacuna da dinâmica quando fazemos o volume do sistema divergir. Para isso, discutiremos uma cota inferior para o inverso da lacuna, mas apenas em um caso particular do modelo, a saber, o GREM com dois níveis, ou seja, $n=2$, sob o regime de temperaturas baixas em fase em cascata (Teorema 6.1.1). 


\section{Capítulo 2}

\section{A dinâmica de Metropolis no GREM}

Os objetivos deste capítulo são dois: descrever e motivar precisamente o processo de Markov - o GREM sujeito à dinâmica de Metropolis - que será o objeto de nosso estudo e formular formalmente o problema que pretendemos resolver ao longo deste trabalho, a saber, estudamos a velocidade com que o processo de Markov converge para o equilíbrio. Essa propriedade é descrita pelo inverso da lacuna espectral, isto é, a diferença entre o primeiro e o segundo autovalor da matriz de probabilidades de transição do correspondente processo estocástico.

O GREM é descrito como um sistema que assume uma configuração que é uma sequência de $N$ elementos pertencentes ao conjunto $\{-1,+1\}$, ou seja, existem $2^{N}$ configurações possíveis cujos Hamiltonianos não são especificados, mas a energia de cada configuração é a soma de $n$ variáveis aleatórias gaussianas.

A evolução do processo dar-se-á em tempo contínuo e os tempos entre as transições podem ser descritos como variáveis aleatórias independentes com distribuição exponencial.

O capítulo está organizado em três seções que descrevemos como segue. Na Seção 2.1 apresentaremos o GREM ao leitor introduzindo ambas versões de sua formulação: a versão original proposta em [Der85], e outra, em [JR06]. Na verdade, como veremos nesta seção, o GREM é mais uma família de modelos que um modelo só, já que depende de duas famílias de parâmetros. Adiantamos que consideraremos uma versão do GREM baseada na definição dada em [JR06]. Encerraremos esta seção introduzindo e motivando alguns dos conceitos fundamentais a respeito do GREM e reunindo alguns dos resultados mais importantes já conhecidos sobre a sua análise. Portanto, a maioria dos conceitos e resultados apresentados nesta seção não serão novos. Eles tem apenas o propósito de uniformizar notações para o contexto do nosso trabalho e, assim, orientar melhor o leitor.

A Seção 2.2 está dedicada à definição da dinâmica de Metropolis para o GREM em um volume finito. Finalizamos o capítulo com a Seção 2.3, em que apresentaremos o resultado principal da 
tese.

\subsection{O Modelo}

Há duas versões equivalentes para a definição do GREM. A versão original formulada em [Der85], e outra em [JR06]. A proposta de [Der85] foi generalizar o REM [Der80] de tal forma que qualquer correlação entre pares de energias pudessem ser levadas em conta, enquanto que a de [JR06] foi motivada pelo desejo de adicionar uma aleatoriedade extra ao nível das correlações.

O modelo é definido da seguinte forma. Seja $N$ um inteiro positivo que eventualmente diverge e representa o volume de um sistema de partículas (que podem ser pessoas, átomos etc.), e que, sem perda de generalidade, enumeramos de 1 a $N$. Vamos considerar que os elementos desse sistema podem assumir dois estados/spins, -1 ou +1 . Então, se denotarmos por $\mathcal{V} \stackrel{\text { def }}{=}\{1, \ldots, N\}$ o conjunto de partículas, as $2^{N}$ configurações possíveis do sistema são os elementos do conjunto

$$
\Omega_{\mathcal{V}} \stackrel{\text { def }}{=}\{-1,+1\}^{\mathcal{V}}
$$

Essas partículas são divididas em grupos de acordo com uma hierarquia em níveis como segue. Seja $n \in \mathbb{N}$ fixado, tal que $n \leq N$, o número de níveis dessa hierarquia. Seguindo [JR06], considere uma partição de $N$ em $n$ inteiros positivos, digamos

$$
N=\sum_{j=1}^{n} V_{j} .
$$

Então, escrevemos

$$
\mathcal{V}=\bigcup_{j=1}^{n} \mathcal{V}_{j}
$$

em que

$$
\mathcal{V}_{j} \stackrel{\text { def }}{=}\left\{1+\sum_{i=1}^{j-1} V_{i}, \ldots, \sum_{i=1}^{j} V_{i}\right\}
$$

é o grupo de partículas do nível $j$ da hierarquia. Em geral, $V_{j} \equiv V_{j, n, N}$ depende dos parâmetros $n$ e $N$, mas, por simplicidade, vamos omitir essa dependência. Naturalmente, $\Omega \mathcal{v}$ pode ser escrito como

$$
\Omega \mathcal{V}=\Omega \mathcal{V}_{1} \times \cdots \times \Omega \mathcal{V}_{n}, \operatorname{com} \Omega \mathcal{V}_{j} \stackrel{\text { def }}{=}\{-1,+1\}^{\mathcal{V}_{j}}
$$

Uma configuração $\sigma \in \Omega_{\mathcal{V}}$ é então da forma

$$
\boldsymbol{\sigma} \stackrel{\text { def }}{=} \sigma_{1}, \ldots, \sigma_{n}
$$

em que $\sigma_{j} \in \Omega_{\mathcal{V}_{j}}$ denota a configuração do $j$-ésimo nível de $\boldsymbol{\sigma}$. 
A fim de obter alguma intuição em (2.1.6), podemos imaginar uma árvore de $n$ níveis. No primeiro nível há $2^{V_{1}}$ ramificações, cada uma representando uma configuração $\sigma_{1} \in \Omega_{\mathcal{V}_{1}}$. Abaixo de cada ramificação $\sigma_{1}$ do nível 1 , há $2^{V_{2}}$ ramificações no nível 2. Cada ramificação abaixo de $\sigma_{1}$ representa uma configuração $\sigma_{1}, \sigma_{2}$ no nível 2, com $\sigma_{2} \in \Omega \mathcal{V}_{2}$. Em geral, abaixo de cada ramificação $\sigma_{1}, \ldots, \sigma_{j}$ do nível $j$, existem $2^{V_{j+1}}$ ramificações que representam, cada uma, as configurações $\sigma_{1}, \ldots, \sigma_{j+1}$ no nível $j+1$, com $\sigma_{j+1} \in \Omega_{\mathcal{V}_{j+1}}$. Uma típica configuração $\boldsymbol{\sigma}=\sigma_{1}, \ldots, \sigma_{n}$ do sistema é então representada unicamente como um caminho nesta árvore que vai da raiz até a folha por meio das ramificações $\sigma_{1}, \sigma_{1}, \sigma_{2}, \ldots, \boldsymbol{\sigma}$. Uma ilustração da ideia segue:

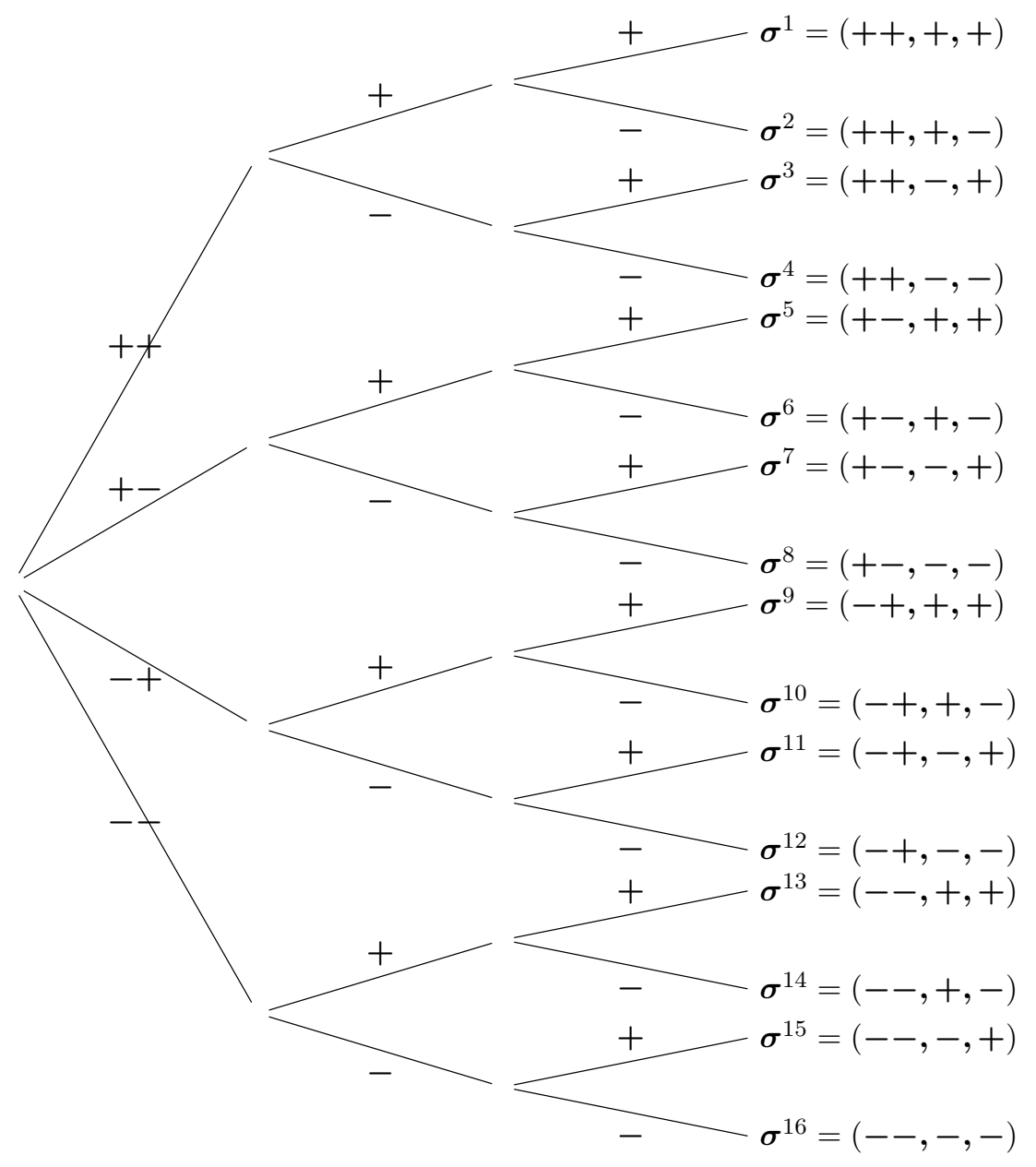

Figura 2.1: Exemplo de uma estrutura de árvore de um GREM com 3 níveis. Neste caso, estamos considerando que $V_{1}=2$ e $V_{2}=V_{3}=1$.

Observação 2.1.1. Na formulação original proposta em [Der85], ao invés de uma partição para 
o inteiro $N$, é considerada uma fatoração do número 2 em $n$ números reais maiores que 1 , isto é,

$$
2=\prod_{j=1}^{n} \alpha_{j}, \operatorname{com} \alpha_{j}>1 .
$$

Para um sistema com $N$ partículas, podemos também definir uma estrutura de árvore considerando uma árvore regular de $n$ níveis com $\alpha_{j}^{N}$ ramificações no $j$-ésimo nível. Obviamente, em geral, $\alpha_{j}^{N}$ não é um inteiro, então tomamos sua parte inteira, ou seja, consideramos apenas $\left\lfloor\alpha_{j}^{N}\right\rfloor$ ramificações no $i$-ésimo nível. O fato é que essa árvore, como é de se esperar, pode não ter as $2^{N}$ ramificações para corresponder exatamente ao espaço de configurações $\Omega_{\mathcal{V}}$. No entanto, como observado em [JR06], fixadas as constantes $\alpha_{1}, \ldots, \alpha_{n}$ satisfazendo (2.1.7), pode-se definir os inteiros $V_{1}, \ldots, V_{n}$ como sendo aqueles tais que

$$
2^{V_{j}} \leq\left\lfloor\alpha_{j}^{N}\right\rfloor \leq 2^{V_{j}+1}
$$

Naturalmente, a condição (2.1.2) não necessariamente é satisfeita com os inteiros $V_{1}, \ldots, V_{n}$ satisfazendo (2.1.8) qualquer que seja $N \in \mathbb{N}$. Mas, observando-se que

$$
\lim _{N \uparrow \infty} \frac{V_{j}}{N}=\frac{\log \alpha_{j}}{\log 2} \stackrel{\text { def }}{=} p_{j} \quad \text { e } \quad \sum_{j=1}^{n} p_{j}=1,
$$

a razão $\frac{1}{N} \sum_{j=1}^{n} V_{j}$ converge à 1 , assim como o lado direito de (2.1.2), quando $N \uparrow \infty$. Em suma, dada a família $\left\{\alpha_{j}\right\}_{j=1}^{n}$, construir os inteiros $V_{1}, \ldots, V_{n}$ que satisfazem (2.1.8) lida com problemas de arredondamento, como por exemplo (2.1.2), que se tornam irrelevantes quando fazemos o volume do sistema divergir.

Notação. Dado $k \in \mathbb{N}$, iremos usar o símbolo $\mathcal{I}_{k}$ para indicar o conjunto $\{1, \ldots, k\}$.

Por razões que serão explicadas no decorrer deste trabalho, consideraremos a família de inteiros $\left\{V_{j}\right\}_{j=1}^{n}$ definidos como segue. Seja $\left\{p_{j}\right\}_{j=1}^{n}$ uma família de números reais positivos tais que

$$
\sum_{j=1}^{n} p_{j}=1
$$

Seja $\mathcal{S} \stackrel{\text { def }}{=} \mathcal{S}(N, n) \uparrow \infty$ quando $N \uparrow \infty$ uma sequência de números não necessariamente inteiros, tal que

$$
\lim _{N \uparrow \infty} \frac{\mathcal{S}}{N}=0,
$$

ou seja, $\mathcal{S}=o(N)$, e para cada $j \in \mathcal{I}_{n}$ vamos escolher

$$
V_{j} \stackrel{\text { def }}{=} \mathrm{v}_{j} \cdot \mathrm{B}
$$


em que

$$
\mathrm{v}_{j}=\mathrm{v}_{j}(N, n) \stackrel{\text { def }}{=}\left\lfloor p_{j} \mathcal{S}\right\rfloor \quad \text { e } \quad \mathrm{B}=\mathrm{B}(N, n) \stackrel{\text { def }}{=}\left\lfloor\frac{N}{\mathcal{S}}\right\rfloor .
$$

Usando que $x-1 \leq\lfloor x\rfloor \leq x, \forall x \in \mathbb{R}$, obtemos então a primeira observação:

$$
p_{j}-\frac{2}{\mathcal{S}} \leq \frac{V_{j}}{N} \leq p_{j}
$$

Em particular,

$$
1-\frac{2 n}{\mathcal{S}} \leq \frac{1}{N} \sum_{j=1}^{n} V_{j} \leq 1 .
$$

Como $n \in \mathbb{N}$ está fixado, a condição $\mathcal{S} \uparrow \infty$ implica que

$$
\lim _{N \uparrow \infty} \frac{1}{N} \sum_{j=1}^{n} V_{j}=1 .
$$

Isto quer dizer que nem sempre teremos $\sum_{j=1}^{n} V_{j}=N$ como em (2.1.2), isto é, o volume do nosso sistema não será necessariamente $N$, mas meramente

$$
V=V(N, n) \stackrel{\text { def }}{=} \sum_{j=1}^{n} V_{j} .
$$

Note que as hipóteses (2.1.11) garantem que não existe uma diferença macroscópica significativa entre o volume $V$ e $N$.

Observação 2.1.2. É importante enfatizar que a formulação do GREM que estamos considerando neste trabalho não é igual à definida em (2.1.2), uma vez que $V$ não é necessariamente igual a $N$. Com respeito a formulação original, devido a (2.1.9), dada a família $\left\{p_{j}\right\}_{j=1}^{n}$, podemos definir a família $\left\{\alpha_{j}\right\}_{j=1}^{n}$ por simplesmente fazer

$$
\alpha_{j}=2^{p_{j}}
$$

Neste caso, o volume do sistema também é $N$, e de novo, $V$ não é necessariamente igual a $N$. Entretanto, como comentado em [JR06], isso não importa, no sentido que quaisquer pequenas diferenças podem ser absorvidas em qualquer um dos $n$ níveis.

Observação 2.1.3. Uma das razões para a escolha em (2.1.12) é que, para todo $j \in \mathcal{I}_{n}, \mathcal{V}_{j}$ pode ser decomposto como

$$
\mathcal{V}_{j}=\bigcup_{i=1}^{\mathrm{B}} \mathcal{V}_{j}(i),
$$

em que $\left|\mathcal{V}_{j}(i)\right|=\mathrm{v}_{j}$. De fato, podemos escrever

$$
\mathcal{V}_{j}(i) \stackrel{\text { def }}{=}\left\{2+(i-1) \mathbf{v}_{j}+\sum_{k=1}^{j-1} V_{k}, \ldots, 1+i \mathbf{v}_{j}+\sum_{k=1}^{j} V_{k}\right\} .
$$

Isto quer dizer que em todos os $n$ níveis da hierarquia existe o mesmo número de subconjuntos, $\mathrm{B}$, que chamamos de blocos, cujos volumes dependem apenas do nível $j$ considerado. 
A cada configuração $\sigma \in \Omega \mathcal{V}$ associaremos sua energia (aleatória), o qual é dada da seguinte forma. Considere uma família, digamos $\mathscr{X}$, de $2^{V_{1}}+2^{V_{1}+V_{2}}+\cdots+2^{V}$ variáveis aleatórias i.i.d. (independentes e identicamente distribuídas) gaussianas padrão definidas sobre o mesmo espaço de probabilidade $(\mathscr{S}, \Sigma, \mathbb{P})$. Frequentemente estas gaussianas são indexadas por $\sigma_{1}, \ldots, \sigma_{j}$, de modo que

$$
\mathscr{X} \stackrel{\text { def }}{=}\left\{X_{\sigma_{1}, \ldots, \sigma_{j}}^{j}: \sigma_{1}, \ldots, \sigma_{j} \in \Omega_{\mathcal{V}_{1}} \times \cdots \times \Omega_{\mathcal{V}_{j}}, j \in \mathcal{I}_{n}\right\}
$$

Note que não estamos denotando a dependência em $N$ explicitamente em $\mathscr{X}$, mas assumimos que o espaço $(\mathscr{I}, \Sigma, \mathbb{P})$ é o mesmo para todo $N$.

Dado $\sigma \in \Omega_{\mathcal{V}}$, vamos denotar por

$$
X_{\boldsymbol{\sigma}} \stackrel{\text { def }}{=}\left(X_{\sigma_{1}}^{1}, X_{\sigma_{1}, \sigma_{2}}^{2}, \ldots, X_{\boldsymbol{\sigma}}^{n}\right)
$$

Seja $\left\{a_{j}\right\}_{j=1}^{n}$ uma família com $n$ números reais positivos tais que

$$
\sum_{j=1}^{n} a_{j}=1
$$

A energia de $\boldsymbol{\sigma}$ é então dada por

$$
H(\boldsymbol{\sigma}) \stackrel{\text { def }}{=} H_{N}(\boldsymbol{\sigma})=-\left\langle\mathfrak{a}, X_{\boldsymbol{\sigma}}\right\rangle \sqrt{V}
$$

em que $\langle\cdot, \cdot\rangle$ é o produto interno usual em $\mathbb{R}^{n} \mathrm{e}$

$$
\mathfrak{a} \stackrel{\text { def }}{=}\left(\sqrt{a_{1}}, \ldots, \sqrt{a_{n}}\right) .
$$

Na literatura a função $H$ é usualmente chamada de Hamiltoniano, então manteremos a tradição aqui.

Observação 2.1.4. É evidente que $H$ é uma variável aleatória no espaço de probabilidade $(\mathscr{S}, \Sigma, \mathbb{P})$. Mais precisamente, como estamos assumindo (2.1.23), obtemos que $H(\boldsymbol{\sigma}) \sim \mathcal{N}(0, V)$ para todo $\sigma \in \Omega_{\mathcal{V}}$, e para $\sigma, \sigma^{\prime} \in \Omega_{\mathcal{V}}$

$$
\frac{1}{V} \mathbb{E}\left[H(\boldsymbol{\sigma}) H\left(\boldsymbol{\sigma}^{\prime}\right)\right]=\sum_{j=1}^{n} a_{j} \mathbb{1}_{\left\{\sigma_{1}=\sigma_{1}^{\prime}\right\}} \cdots \mathbb{1}_{\left\{\sigma_{j}=\sigma_{j}^{\prime}\right\}}=\sum_{j=1}^{R\left(\boldsymbol{\sigma}, \boldsymbol{\sigma}^{\prime}\right)} a_{j},
$$

em que,

$$
R\left(\boldsymbol{\sigma}, \boldsymbol{\sigma}^{\prime}\right) \stackrel{\text { def }}{=} \max \left\{j \in \mathcal{I}_{n}: \sigma_{1}, \ldots, \sigma_{j}=\sigma_{1}^{\prime}, \ldots, \sigma_{j}^{\prime}\right\}
$$

Segue então de (2.1.26) que se $\boldsymbol{\sigma}$ e $\boldsymbol{\sigma}^{\prime}$ são configurações que diferem no primeiro nível, isto é, $\mathbb{1}_{\left\{\sigma_{1}=\sigma_{1}^{\prime}\right\}}=0$, então $H(\boldsymbol{\sigma})$ e $H\left(\boldsymbol{\sigma}^{\prime}\right)$ são gaussianas independentes. 
Definimos a medida de probabilidade (Gibbs) $\mu$ sobre $\Omega_{\mathcal{V}}$ com Hamiltoniano $H$ por

$$
\mu(\boldsymbol{\sigma}) \stackrel{\text { def }}{=} \mu_{N, n}^{\beta}(\boldsymbol{\sigma})=\frac{e^{-\beta H(\boldsymbol{\sigma})}}{Z}
$$

em que

$$
Z \stackrel{\text { def }}{=} Z_{N, n}(\beta)=\sum_{\boldsymbol{\sigma} \in \Omega_{\mathcal{V}}} e^{-\beta H(\boldsymbol{\sigma})}
$$

é a função de partição a volume finito $V$ e $\beta>0$ é o parâmetro inverso da temperatura. Como é usual, a função

$$
F_{N, n}(\beta) \stackrel{\text { def }}{=} \frac{1}{V} \log Z
$$

denota a energia livre do sistema a volume finito $V$. Note que, como $H$, as quantidades $\mu, Z$ e $F_{N, n}$ são sequências de variáveis aleatórias em $N$ sobre o espaço de probabilidade $(\mathscr{P}, \Sigma, \mathbb{P})$.

Sob a definição original de Derrida [Der85], é bem conhecido de [CCP87] (Teorema 2.1) que para todo $\beta>0$ a energia livre do GREM existe em ambos, $\mathbb{P}$-quase certamente e $L_{p}(\mathscr{S}, \Sigma, \mathbb{P})$ para $1 \leq p<\infty$. Sobre esse resultado, cujas ideias serão úteis neste trabalho, a estratégia principal foi similar à usada para o REM em [OP84]. Nesse caso, o ingrediente crucial foi que, para $N$ grande o suficiente, o máximo de uma sequência de $2^{N}$ variáveis aleatórias gaussianas independentes é quase certamente limitado.

No caso do GREM, como cada função energia é a soma de $n$ variáveis aleatórias gaussianas i.i.d., então foi exibida uma região convexa compacta em $\mathbb{R}^{n}$ em que, com uma altíssima probabilidade, os $2^{N}$ pontos associados com a sequência $H\left(\boldsymbol{\sigma}^{1}\right), H\left(\boldsymbol{\sigma}^{2}\right), \ldots, H\left(\boldsymbol{\sigma}^{2^{N}}\right)$ moram (cf. Proposição 3.1). Sob nosso cenário, dado $\delta>0$, essa região convexa é escrita como

$$
\mathcal{A}_{n}(N, \delta) \stackrel{\text { def }}{=}\left\{\forall j \in \mathcal{I}_{n}, \forall \sigma_{1}, \ldots, \sigma_{j} \in \prod_{i=1}^{j} \Omega_{\mathcal{V}_{j}}, \sum_{i=1}^{j}\left(X_{\sigma_{1}, \ldots, \sigma_{i}}^{i}\right)^{2} \leq \beta_{\star}^{2}(1+\delta) \sum_{i=1}^{j} V_{i}\right\},
$$

em que

$$
\beta_{\star} \stackrel{\text { def }}{=} \sqrt{2 \log 2}
$$

Note que o conjunto $\mathcal{A}_{n}(N, \delta)$ é a interseção de uma esfera $n$-dimensional com vários cilindros cujas bases são de dimensão $1,2, \ldots, n-1$.

O resultado seguinte é análogo à Proposição 3.1 em [CCP87].

Proposição 2.1.1. Para todo $\delta>0$,

$$
\mathbb{P}\left[\liminf _{N \uparrow \infty} \mathcal{A}_{n}(N, \delta)\right]=\mathbb{P}\left[\bigcup_{M \geq 1} \bigcap_{N \geq M} \mathcal{A}_{n}(N, \delta)\right]=1 .
$$


Demonstração. Primeiro, note que

$$
\mathbb{P}\left[\bigcup_{M \geq 1} \bigcap_{N \geq M} \mathcal{A}_{n}(N, \delta)\right]=1-\mathbb{P}\left[\mathcal{A}_{n}^{c}(N) \text { i.v. }\right]
$$

em que i.v. significa infinitas vezes. Pelo Primeiro Lema de Borel-Cantelli é suficiente então provarmos que

$$
\sum_{N \geq 1} \mathbb{P}\left[\mathcal{A}_{n}^{c}(N, \delta)\right]<\infty
$$

Por definição, temos que

$$
\mathbb{P}\left[\mathcal{A}_{n}^{c}(N, \delta)\right]=\mathbb{P}\left[\exists i \in \mathcal{I}_{n}: \max _{\sigma_{1} \cdots \sigma_{i}} \sum_{j=1}^{i}\left(X_{\sigma_{1} \cdots \sigma_{j}}^{j}\right)^{2}>\beta_{\star}^{2}(1+\delta) \sum_{j=1}^{i} V_{j}\right] .
$$

Obviamente,

$$
\mathbb{P}\left[\mathcal{A}_{n}^{c}(N, \delta)\right] \leq \sum_{i=1}^{n} \mathbb{P}\left[G_{i}(N)\right]
$$

em que $G_{i}(N)$ denota o evento

$$
G_{i}(N)=\left\{\max _{\sigma_{1} \cdots \sigma_{i}} \sum_{j=1}^{i}\left(X_{\sigma_{1} \cdots \sigma_{j}}^{j}\right)^{2}>\beta_{\star}^{2}(1+\delta) \sum_{j=1}^{i} V_{j}\right\} .
$$

Da desigualdade exponencial de Markov e estimativas padrão, obtemos que

$$
\begin{aligned}
\mathbb{P}\left[G_{i}(N)\right] & \leq 2^{\sum_{j=1}^{i} V_{j}} e^{-t(1+\delta) \frac{\beta_{\star}^{2}}{2} \sum_{j=1}^{i} V_{j}}(1-t)^{-\frac{i}{2}} \\
& =e^{(1-t(1+\delta)) \frac{\beta_{\star}^{2}}{2} \sum_{j=1}^{i} V_{j}}(1-t)^{-\frac{i}{2}}
\end{aligned}
$$

Escolhendo $t=1-c$, com

$$
0<c<1-\frac{1+\frac{\delta}{2}}{1+\delta}
$$

obtemos então que

$$
\mathbb{P}\left[\mathcal{A}_{n}^{c}(N, \delta)\right] \leq \sum_{i=1}^{n} c^{-\frac{i}{2}} e^{-\frac{\delta \beta_{\star}^{2}}{4} \sum_{j=1}^{i} V_{j}} .
$$

Como as constantes $\delta, \beta_{\star}$ e $c$ são fixas e $V_{j} \uparrow \infty$ quando $N \uparrow \infty$, segue que

$$
\sum_{N \geq 1} \mathbb{P}\left[\mathcal{A}_{n}^{c}(N, \delta)\right]<\infty
$$

como queríamos.

Apesar de não ser difícil adaptar os demais resultados de [CCP87] (cf. Proposições 3.3 e 3.5) para verificar a existência da termodinâmica do GREM que estamos considerando, neste trabalho é mais conveniente usar o seguinte. 
Teorema 2.1.1 (Jana e Rao [JR06]). Considere um GREM com $n$ níveis e volume $N=$ $\sum_{j=1}^{n} k_{j, N}$ e assuma que para todo $j \in \mathcal{I}_{n}$,

$$
\lim _{N \uparrow \infty} \frac{k_{j, N}}{N}=p_{j}>0 .
$$

Então, com $\mathbb{P}$-probabilidade 1,

$$
F_{n}(\beta)=\lim _{N \uparrow \infty} \frac{1}{N} \log Z=\frac{\beta_{\star}^{2}}{2}+\frac{\beta^{2}}{2}-\frac{1}{2}\left\|\mathfrak{m}^{*}-\mathfrak{m}\right\|^{2},
$$

em que $\|\cdot\|$ é a norma induzida pelo produto interno usual em $\mathbb{R}^{n}$,

$$
\mathfrak{m}^{*} \stackrel{\text { def }}{=} \mathfrak{m}^{*}(\beta)=\beta \mathfrak{a},
$$

em que a está dado em (2.1.25) e $\mathfrak{m}$ é o ponto do convexo compacto $\Psi \subset \mathbb{R}^{n}$, o subconjunto de $\mathbb{R}^{n}$ definido como

$$
\Psi \stackrel{\text { def }}{=}\left\{x \in \mathbb{R}^{n}: \sum_{i=1}^{j} x_{i}^{2} \leq P_{j}, \forall j=1, \ldots, n\right\}
$$

em que

$$
P_{j} \stackrel{\text { def }}{=} \beta_{\star}^{2} \sum_{i=1}^{j} p_{i},
$$

que minimiza a distância entre $\mathfrak{m}^{*}$ e $\Psi$, isto é,

$$
\mathrm{d}\left(\mathfrak{m}^{*}, \Psi\right)=\inf _{x \in \Psi}\left\|\mathfrak{m}^{*}-x\right\|=\left\|\mathfrak{m}^{*}-\mathfrak{m}\right\| .
$$

Corolário 2.1.1. Considere o GREM com $n$ níveis e volume $V=\sum_{j=1}^{n} V_{j}$, em que $V_{j}$ é como definido em (2.1.12). Então, para todo $\beta>0$, com $\mathbb{P}$-probabilidade 1,

$$
\lim _{N \uparrow \infty} F_{N, n}(\beta)=F_{n}(\beta)
$$

Demonstração. É imediato de (2.1.14) e do Teorema 2.1.1.

Note que uma fórmula explícita para a função $F_{n}(\beta)$ é obtida se, no Teorema 2.1.1, exibirmos as coordenadas do ponto $\mathfrak{m}$. Os primeiros a fazerem isso foram Capocaccia et al. em [CCP87]. Depois vieram Dorlas e Dukes e exibiram em [DD02] uma expressão explícita para a energia livre usando a simples desigualdade de Cauchy-Schwarz. Por questões de formulação, indicaremos aqui a fórmula que foi dada em [JR06], mas que, nas palavras dos próprios autores, é essencialmente a mesma dada em [CCP87] e [DD02].

Para todo $1 \leq j \leq k \leq n$, defina os números $B_{j, k}$ por

$$
B_{j, k} \stackrel{\text { def }}{=} \beta_{\star} \sqrt{\frac{\sum_{i=j}^{k} p_{i}}{\sum_{i=j}^{k} a_{i}}} .
$$


Seja

$$
\begin{aligned}
& \beta_{1} \stackrel{\text { def }}{=} \min _{1 \leq k \leq n} B_{1, k} \quad J_{1}^{*} \stackrel{\text { def }}{=} \max \left\{1 \leq i \leq n: B_{1, i}=\beta_{1}\right\} ; \\
& \beta_{2} \stackrel{\text { def }}{=} \min _{J_{1}^{*}<k \leq n} B_{J_{1}^{*}+1, k} \quad J_{2}^{*} \stackrel{\text { def }}{=} \max \left\{J_{1}^{*}<i \leq n: B_{J_{1}^{*}+1, i}=\beta_{2}\right\},
\end{aligned}
$$

mais geralmente,

$$
\beta_{\ell+1} \stackrel{\text { def }}{=} \min _{J_{\ell}^{*}<k \leq n} B_{J_{\ell}^{*}+1, k} \quad J_{\ell+1}^{*} \stackrel{\text { def }}{=} \max \left\{J_{\ell}^{*}<i \leq n: B_{J_{\ell}^{*}+1, i}=\beta_{\ell+1}\right\} .
$$

Obviamente, para algum inteiro $\ell_{n} \in \mathcal{I}_{n}$, temos que $J_{\ell_{n}}^{*}=n$.

Usando a convenção que $J_{0}^{*}=\beta_{0}=0$ e $\beta_{\ell_{n}+1}=\infty$, observe que as sequências $\left\{J_{\ell}^{*}\right\}_{\ell=1}^{\ell_{n}}$ e $\left\{\beta_{\ell}\right\}_{\ell=1}^{\ell_{n}}$ são tais que

$$
0=J_{0}^{*}<J_{1}^{*}<\cdots<J_{\ell_{n}}^{*}=n
$$

$\mathrm{e}$

$$
0=\beta_{0}<\beta_{1}<\cdots<\beta_{\ell_{n}}<\beta_{\ell_{n}+1}=\infty
$$

Então, se $\beta_{\ell} \leq \beta<\beta_{\ell+1}$ para algum $\ell \in\left\{1, \ldots, \ell_{n}\right\}$, o ponto $\mathfrak{m} \in \Psi$ é definido por

$$
\mathfrak{m}_{i} \stackrel{\text { def }}{=} \begin{cases}\beta_{k} \sqrt{a_{i}}, & \text { se } i \in\left\{J_{k-1}^{*}+1, \ldots, J_{k}^{*}\right\}, \text { para algum } k=1, \ldots, \ell ; \\ \beta \sqrt{a_{i}}, & \text { se } i \in\left\{J_{\ell}^{*}+1, \ldots, n\right\},\end{cases}
$$

e a função $F_{n}(\beta)$ fica explicitamente dada por

$$
F_{n}(\beta)= \begin{cases}\frac{\beta_{\star}^{2}}{2}+\frac{\beta^{2}}{2} & \text { se } \beta<\beta_{1} ; \\ \beta \sum_{i=1}^{\ell} \beta_{i} \sum_{k=J_{i-1}^{*}+1}^{J_{i}^{*}} a_{k}+\frac{1}{2} \sum_{k=J_{\ell}^{*}+1}^{n}\left(\beta_{\star}^{2} p_{k}+\beta^{2} a_{k}\right), & \text { se } \beta_{\ell} \leq \beta<\beta_{\ell+1}, \\ \beta \sum_{i=1}^{\ell_{n}} \beta_{i} \sum_{k=J_{i-1}^{*}+1}^{J_{i}^{*}} a_{k} & \forall 1 \leq \ell \leq \ell_{n}-1 ; \\ & \text { se } \beta \geq \beta_{\ell_{n}} .\end{cases}
$$

\subsection{A dinâmica}

A fim de formular a dinâmica que consideramos neste trabalho, Metropolis, é conveniente olhar o espaço de configurações $\Omega_{\mathcal{V}}$ como o hipercubo $V$-dimensional obtido pela adição de elos entre configurações vizinhas próximas, aquelas configurações que diferem em apenas um spin, equipado com a métrica usual, a distância de Hamming, a qual é definida por

$$
\mathrm{d}_{\mathrm{H}}\left(\boldsymbol{\sigma}, \boldsymbol{\sigma}^{\prime}\right) \stackrel{\text { def }}{=} \frac{1}{2} \sum_{i=1}^{V}\left|\boldsymbol{\sigma}(i)-\boldsymbol{\sigma}^{\prime}(i)\right| .
$$


Usaremos o símbolo $\mathcal{E}$ para denotar o conjunto de elos do hipercubo $\Omega \mathcal{V}$, isto é,

$$
\mathcal{E} \stackrel{\text { def }}{=}\left\{\left(\boldsymbol{\sigma}, \boldsymbol{\sigma}^{\prime}\right) \in \Omega_{\mathcal{V}} \times \Omega_{\mathcal{V}}: \mathrm{d}_{\mathrm{H}}\left(\boldsymbol{\sigma}, \boldsymbol{\sigma}^{\prime}\right)=1\right\}
$$

Usaremos também o termo "vértice" para nos referir aos elementos de $\Omega_{\mathcal{V}}$.

Para uma dada realização de $\mathscr{X}$, considere uma cadeia de Markov a tempo discreto com matriz de probabilidades de transição $\mathrm{P} \stackrel{\text { def }}{=}\left(\mathrm{P}\left(\boldsymbol{\sigma}, \boldsymbol{\sigma}^{\prime}\right)\right)_{\boldsymbol{\sigma}, \boldsymbol{\sigma}^{\prime} \in \Omega_{\mathcal{V}}}$ tipo-Metropolis, isto é, para todo $\sigma, \sigma^{\prime} \in \Omega \mathcal{V}$

$$
\mathrm{P}\left(\boldsymbol{\sigma}, \boldsymbol{\sigma}^{\prime}\right) \stackrel{\text { def }}{=} \begin{cases}\frac{1}{V} \exp \left\{-\beta\left[H\left(\boldsymbol{\sigma}^{\prime}\right)-H(\boldsymbol{\sigma})\right]^{+}\right\}, & \text {se }\left(\boldsymbol{\sigma}, \boldsymbol{\sigma}^{\prime}\right) \in \mathcal{E} ; \\ 1-\sum_{\boldsymbol{\sigma}^{\prime \prime} \neq \boldsymbol{\sigma}} \mathrm{P}\left(\boldsymbol{\sigma}, \boldsymbol{\sigma}^{\prime \prime}\right), & \text { se } \boldsymbol{\sigma}=\boldsymbol{\sigma}^{\prime} \\ 0, & \text { caso contrário, }\end{cases}
$$

em que $H$ é o Hamiltoniano do GREM definido em (2.1.24), $\beta>0$ é o parâmetro inverso da temperatura e $a^{+} \stackrel{\text { def }}{=} \max \{a, 0\}$. Naturalmente, podemos associar a matriz $\mathrm{P}$ a matriz de taxas de transição $\left(c\left(\boldsymbol{\sigma}, \boldsymbol{\sigma}^{\prime}\right)\right)_{\boldsymbol{\sigma}, \boldsymbol{\sigma}^{\prime} \in \Omega_{\mathcal{V}}}$ a qual é única e tal que

$$
c\left(\boldsymbol{\sigma}, \boldsymbol{\sigma}^{\prime}\right) \stackrel{\text { def }}{=} \frac{1}{V} \exp \left\{-\beta\left[H\left(\boldsymbol{\sigma}^{\prime}\right)-H(\boldsymbol{\sigma})\right]^{+}\right\} \mathbb{1}\left\{\left(\boldsymbol{\sigma}, \boldsymbol{\sigma}^{\prime}\right) \in \mathcal{E}\right\}, \quad \forall \boldsymbol{\sigma}, \boldsymbol{\sigma}^{\prime} \in \Omega_{\mathcal{V}} .
$$

Chamamos de dinâmica de Metropolis para o GREM ao processo Markoviano a tempo contínuo $Y=\left(Y_{N}(t), t \geq 0\right)$ sobre o espaço de estados $\Omega_{\mathcal{V}}$ gerado pelo operador de Markov $\mathscr{L}$ aplicado sobre funções $f: \Omega \mathcal{V} \rightarrow \mathbb{R}$ e definido como

$$
\mathscr{L} f(\boldsymbol{\sigma}) \stackrel{\text { def }}{=} \sum_{\boldsymbol{\sigma}^{\prime} \in \Omega} c\left(\boldsymbol{\sigma}, \boldsymbol{\sigma}^{\prime}\right)\left[f(\boldsymbol{\sigma})-f\left(\boldsymbol{\sigma}^{\prime}\right)\right], \quad \boldsymbol{\sigma} \in \Omega \mathcal{V} .
$$

Observação 2.2.1. Usando as condições do balanço detalhado, não é difícil verificar que $Y$ é uma cadeia de Markov reversível com respeito a medida de Gibbs $\mu$ definida em (2.1.28). Isto significa que $\mu$ é também estacionária e ergódica para esta dinâmica.

O semigrupo Markoviano a tempo contínuo $\mathrm{P}_{t}$ associado com a matriz de transição $\mathrm{P}$ é definido por

$$
\mathrm{P}_{t} \stackrel{\text { def }}{=} e^{-t} \sum_{k=0}^{\infty} \frac{t^{k} \mathrm{P}^{k}}{k !} .
$$

Consequentemente, o núcleo de transição de $Y$ é dado por

$$
\mathrm{P}_{t}\left(\boldsymbol{\sigma}, \boldsymbol{\sigma}^{\prime}\right) \stackrel{\text { def }}{=} e^{-t} \sum_{k=0}^{\infty} \frac{t^{k}}{k !} \mathrm{P}^{k}\left(\boldsymbol{\sigma}, \boldsymbol{\sigma}^{\prime}\right), \quad \forall \boldsymbol{\sigma}, \boldsymbol{\sigma}^{\prime} \in \Omega \mathcal{V} .
$$

Observação 2.2.2. Uma característica essencial das taxas de transição (2.2.3) é que elas favorecem trajetórias que diminui o Hamiltoniano. Não apenas isso, o fato das energias $\{H(\boldsymbol{\sigma}): \boldsymbol{\sigma} \in$ 
$\left.\Omega_{\mathcal{V}}\right\}$ serem a soma de $n$ variáveis aleatórias gaussianas independentes que carregam a estrutura em níveis dos elementos de $\Omega \mathcal{V}$ faz com que as taxas favoreçam trajetórias em que os estados visitados não sejam de níveis muito diferentes, na verdade, que discordem apenas no $n$-ésimo nível. O outro lado dessa característica é que ela também faz o estudo da dinâmica muito difícil porque tornam os saltos da cadeia um objeto complicado de se analisar, já que dependem da aleatoriedade do Hamiltoniano.

\subsection{Resultado Principal}

A partir de agora consideraremos o GREM como um sistema fora do equilíbrio submetido à dinâmica de Metropolis em volume finito $V$. Neste trabalho queremos estender para o GREM o que foi feito por [FIKP98] para o caso do REM. Mais precisamente, estimaremos o inverso da lacuna espectral da dinâmica, isto é, o inverso da diferença entre o primeiro e o segundo autovalor do gerador infinitesimal $\mathscr{L}$ do processo Markoviano $Y$ (ou da matriz de probabilidades de transição P, a lacuna é a mesma) quando o volume tende ao infinito.

É evidente que o primeiro (maior) autovalor de P é 1, e como P é irredutível*, as funções constantes são as únicas autofunções com autovalor 1. Recorrendo agora a usual caracterização de autovalores, conhecida como Princípio min-max de Courant-Fischer (veja, por exemplo, [HHJ90], p. 176; recomendamos também ver [DS91], onde uma descrição do princípio sob um contexto semelhante ao deste trabalho é dada), temos que o inverso da diferença entre 1 e o segundo autovalor de $\mathrm{P}$, que denotamos por $\tau_{N}$, é dado por

$$
\tau_{N}=\sup \left\{\tau_{N}(\phi): \phi \text { não constante }\right\}
$$

em que $\phi$ é uma função real definida sobre $\Omega \mathcal{V}$ e

$$
\tau_{N}(\phi) \stackrel{\text { def }}{=} \frac{\operatorname{Var}_{\mu}(\phi)}{\mathscr{E}(\phi, \phi)}
$$

sendo

$$
\operatorname{Var}_{\mu}(\phi) \stackrel{\text { def }}{=} \frac{1}{2} \sum_{\boldsymbol{\sigma}, \boldsymbol{\sigma}^{\prime}}\left(\phi(\boldsymbol{\sigma})-\phi\left(\boldsymbol{\sigma}^{\prime}\right)\right)^{2} \mu(\boldsymbol{\sigma}) \mu\left(\boldsymbol{\sigma}^{\prime}\right)
$$

a variância de $\phi$ com respeito a medida de equilíbrio $\mu$, e

$$
\mathscr{E}(\phi, \phi) \stackrel{\text { def }}{=} \frac{1}{2} \sum_{\boldsymbol{\sigma}, \boldsymbol{\sigma}^{\prime}}\left(\phi(\boldsymbol{\sigma})-\phi\left(\boldsymbol{\sigma}^{\prime}\right)\right)^{2} \mu(\boldsymbol{\sigma}) \mathrm{P}\left(\boldsymbol{\sigma}, \boldsymbol{\sigma}^{\prime}\right),
$$

a forma de Dirichlet associado ao semigrupo de Markov $\mathrm{P}_{t}$.

${ }^{*}$ Dizemos que uma matriz de probabilidades de transição $\mathrm{P}=\left(\mathrm{P}\left(\boldsymbol{\sigma}, \boldsymbol{\sigma}^{\prime}\right)\right)_{\boldsymbol{\sigma}, \boldsymbol{\sigma}^{\prime} \in \Omega_{\mathcal{V}}}$ é irredutível se para todo $\boldsymbol{\sigma}, \boldsymbol{\sigma}^{\prime} \in \Omega_{\mathcal{V}}$ existe $k \in \mathbb{N}$ tal que $\mathrm{P}^{k}\left(\boldsymbol{\sigma}, \boldsymbol{\sigma}^{\prime}\right)>0$. 
Ainda em [DS91] (cf. Proposição 3), para a distância em variação total ${ }^{\ddagger}$, vale a seguinte desigualdade:

$$
4\left\|\mathrm{P}_{t}(\boldsymbol{\sigma}, \cdot)-\mu(\cdot)\right\|_{\mathrm{var}}^{2} \leq \frac{1-\mu(\boldsymbol{\sigma})}{\mu(\boldsymbol{\sigma})} \exp \left\{-2 t \tau_{N}^{-1}\right\}
$$

Isto significa que mesmo que se esteja interessado em exibir um comportamento assintótico exato para o inverso da lacuna, se alguém deseja usar a desigualdade em (2.3.6), então é meramente uma cota superior para $\tau_{N}$ que é importante.

O resultado mais importante deste trabalho é o seguinte:

Teorema 2.3.1. Para todo $\beta>0$, com $\mathbb{P}$-probabilidade 1 ,

$$
\limsup _{N \uparrow \infty} \frac{1}{N} \log \tau_{N} \leq \beta \sum_{\ell=1}^{\ell_{n}} \beta_{\ell} \sum_{k=J_{\ell-1}^{*}+1}^{J_{\ell}^{*}} a_{k} .
$$

Demonstração. Capítulos 3, 4 e 5.

Observação 2.3.1. Note que a expressão do lado direito em (2.3.7), vista como função de $\beta$, é a mesma expressão que descreve a energia livre do GREM em baixas temperaturas (cf. (2.1.56)). Como consequência deste fato, a Proposição 4.2 em [FIKP98] pode ser obtida como corolário do nosso teorema. Com efeito, basta considerarmos o caso particular $n=1$. Na verdade, a característica mais importante do Teorema (2.3.1) é que a desigualdade 2.3 .7 vale para todo $\beta>0$, todo $n \in \mathbb{N}$ fixo e para quaisquer escolhas de famílias de números reais positivos $\left\{p_{j}\right\}_{j=1}^{n}$ e $\left\{a_{j}\right\}_{j=1}^{n}$ satisfazendo a condição que

$$
\sum_{j=1}^{n} p_{j}=\sum_{j=1}^{n} a_{j}=1
$$

${ }^{\ddagger}$ A distância em variação total entre duas medidas de probabilidade $\mu$ e $\nu$ sobre $\Omega \mathcal{V}$ é definida como

$$
\|\mu-\nu\|_{\text {var }} \stackrel{\text { def }}{=} \max _{A \subset \Omega \mathcal{V}}|\mu(A)-\nu(A)|=\frac{1}{2} \sum_{\boldsymbol{\sigma} \in \Omega_{\mathcal{V}}}|\mu(\boldsymbol{\sigma})-\nu(\boldsymbol{\sigma})| .
$$




\section{Capítulo 3}

\section{Estratégia Geral}

Este é o primeiro capítulo, de uma série de três, dedicado a provar o Teorema 2.3.1. Nosso primeiro passo, talvez o mais importante, é escolher a técnica que usaremos para provar o teorema. Existem diversas na literatura variando entre analíticas, geométricas etc. (veja por exemplo [SC97]). No entanto, algumas informações precisam ser levadas em conta para essa decisão. Por exemplo, devemos escolher uma técnica de estimação que não demande informações muito precisas sobre a geometria do Hamiltoniano $H$, uma vez que essa informação é usualmente nula em modelos de vidros de spins, mas que, por outro lado, explore a geometria do grafo induzido pelas probabilidades de transição $\mathrm{P}(\cdot, \cdot)$ da cadeia. Além disso, a técnica não pode levar em conta qualquer dependência da configuração inicial do sistema, já que queremos derivar uma estimativa para o inverso da lacuna $\tau_{N}$ que seja válida mesmo quando o processo inicia da pior configuração inicial possível, a de energia mínima.

É bem conhecido que cotas para o inverso da lacuna de um processo de Markov reversível podem ser obtidas usando desigualdades do tipo Poincaré, isto é, desigualdades do tipo

$$
\forall \phi, \quad \operatorname{Var}_{\mu}(\phi) \leq \mathrm{K} \cdot \mathscr{E}(\phi, \phi)
$$

para algum constante positiva $\mathrm{K}$, pois, da definição (2.3.1), a desigualdade acima é equivalente a

$$
\tau_{N} \leq \mathrm{K}
$$

O propósito deste capítulo é apresentar a ferramenta que escolhemos: a desigualdade de Poincaré introduzida por Sinclair [Sin91] (cf. Teorema 5). Trata-se de uma versão modificada da desigualdade de Poincaré derivada por Diaconis e Stroock [DS91], uma desigualdade baseada em uma técnica de caminhos criada para se obter estimativas para o segundo maior autovalor e para a lacuna espectral de uma cadeia de Markov reversível. 
O capítulo está organizado da seguinte forma. A Seção 3.1 está dedicada simplesmente à descrição da desigualdade de Poincaré dada em [Sin91] para o contexto da dinâmica de Metropolis para o GREM. Na última seção, a Seção 3.2, motivaremos e construiremos cuidadosamente a coleção de caminhos com a qual derivamos a cota superior para o inverso da lacuna. Encerraremos o capítulo obtendo as primeiras estimativas e, em um breve argumento, mostraremos que a desigualdade em (2.3.7) é verdadeira quando assumimos um regime de temperaturas baixas para o sistema.

\subsection{A Desigualdade de Poincaré}

O objetivo desta seção é descrever precisamente a desigualdade de Poincaré dada por [Sin91]. Ela é, em outras palavras, a desigualdade derivada por [DS91] expressada em termos dos chamados caminhos canônicos de Jerrum e Sinclair [SJ89] (cf. Proposição 1' em [DS91]). Entendemos aqui por caminho canônico entre dois vértices distintos de $\Omega_{\mathcal{V}}$ um caminho que usa apenas transições válidas de $\mathrm{P}$.

A descrição da desigualdade segue. O primeiro ingrediente é um grafo com vértices em $\Omega_{\mathcal{V}}$. Escolhemos o próprio $\Omega_{\mathcal{V}}$ visto como o hipercubo $V$-dimensional. Para cada par de vértices distintos $\boldsymbol{\eta}, \boldsymbol{\eta}^{\prime} \in \Omega_{\mathcal{V}}$, escolhemos um caminho $\gamma_{\boldsymbol{\eta}, \boldsymbol{\eta}^{\prime}}$ indo de $\boldsymbol{\eta}$ para $\boldsymbol{\eta}^{\prime}$ no grafo $\Omega_{\mathcal{V}}$. Um caminho $\gamma$ em $\Omega_{\mathcal{V}}$ é uma sequência de vértices

$$
\gamma \stackrel{\text { def }}{=}\left(\boldsymbol{\sigma}_{0}, \ldots, \boldsymbol{\sigma}_{k}\right), \operatorname{com}\left(\boldsymbol{\sigma}_{i-1}, \boldsymbol{\sigma}_{i}\right) \in \mathcal{E}, \quad i=1, \ldots, k
$$

em que $\mathcal{E}$ está definido em (2.2.2). Equivalentemente, $\gamma$ pode ser visto como uma sequência de elos

$$
\gamma=\left(e_{1}, \ldots, e_{k}\right), \text { com } e_{i} \stackrel{\text { def }}{=}\left(\boldsymbol{\sigma}_{i-1}, \boldsymbol{\sigma}_{i}\right), \quad i=1, \ldots, k
$$

O caminho pode ter vértices repetidos, mas um dado elo aparece no máximo uma vez em $\gamma_{\boldsymbol{\eta}, \boldsymbol{\eta}^{\prime}}$ (os caminhos são auto-evitantes). Denotemos por

$$
\Gamma \stackrel{\text { def }}{=}\left\{\gamma_{\boldsymbol{\eta}, \boldsymbol{\eta}^{\prime}}: \boldsymbol{\eta}, \boldsymbol{\eta}^{\prime} \in \Omega_{\mathcal{V}}\right\}
$$

o conjunto dos caminhos - exatamente um para cada par de vértices distintos $\boldsymbol{\eta}, \boldsymbol{\eta}^{\prime} \in \Omega_{\mathcal{V}}$.

Com tal família de caminhos, denotando por

$$
\mathrm{K} \stackrel{\text { def }}{=} \mathrm{K}_{N}(\Gamma)=\max _{e \in \mathcal{E}} \frac{1}{Q(e)} \sum_{\gamma_{\boldsymbol{\eta}, \boldsymbol{\eta}^{\prime}} \ni e}\left|\gamma_{\boldsymbol{\eta}, \boldsymbol{\eta}^{\prime}}\right| \mu(\boldsymbol{\eta}) \mu\left(\boldsymbol{\eta}^{\prime}\right)
$$

em que o máximo está sobre todos os elos $e=\left(\boldsymbol{\sigma}, \boldsymbol{\sigma}^{\prime}\right) \in \mathcal{E}, Q(e) \stackrel{\text { def }}{=} \mu(\boldsymbol{\sigma}) \mathrm{P}\left(\boldsymbol{\sigma}, \boldsymbol{\sigma}^{\prime}\right)$, o somatório está sobre todos os caminhos $\gamma_{\boldsymbol{\eta}, \boldsymbol{\eta}^{\prime}}$ em $\Gamma$ indexados por seus extremos $\boldsymbol{\eta}$ e $\boldsymbol{\eta}^{\prime}$ que passam pelo 
elo e e $\left|\gamma_{\boldsymbol{\eta}, \boldsymbol{\eta}^{\prime}}\right|$ denota o comprimento (número de elos) do caminho $\gamma_{\boldsymbol{\eta}, \boldsymbol{\eta}^{\prime}}$, segue do Teorema 5 em [Sin91] (ou Proposição 1' em [DS91]) que

$$
\tau_{N} \leq \mathrm{K}
$$

Para a dinâmica de Metropolis em (2.2.3), dado um elo $e=\left(\boldsymbol{\sigma}, \boldsymbol{\sigma}^{\prime}\right)$, obtemos que

$$
\frac{1}{Q(e)}=V Z e^{\beta\left[H(\boldsymbol{\sigma}) \vee H\left(\boldsymbol{\sigma}^{\prime}\right)\right]}
$$

em que $a \vee b=\max \{a, b\}$. Então, de (2.1.28) e (3.1.6), a quantidade K pode ser escrita mais explicitamente como

$$
\mathrm{K}=\frac{V}{Z} \max _{e=\left(\boldsymbol{\sigma}, \boldsymbol{\sigma}^{\prime}\right)} e^{\beta\left[H(\boldsymbol{\sigma}) \vee H\left(\boldsymbol{\sigma}^{\prime}\right)\right]} \sum_{\gamma_{\boldsymbol{\eta}, \boldsymbol{\eta}^{\prime}} \ni e}\left|\gamma_{\boldsymbol{\eta}, \boldsymbol{\eta}^{\prime}}\right| e^{-\beta\left[H(\boldsymbol{\eta})+H\left(\boldsymbol{\eta}^{\prime}\right)\right]} .
$$

Note que a qualidade da estimativa em (3.1.7) depende do quão sábia é a escolha do conjunto de caminhos. Desta forma, nosso próximo passo será então construir uma família de caminhos sobre $\Omega_{\mathcal{V}}$ que seja completa, isto é, todo par de vértices distintos de $\Omega_{\mathcal{V}}$ é extremo de algum caminho da família, e que todos os caminhos sejam auto-evitantes.

\section{$3.2 \quad$ O Conjunto de Caminhos}

Como em [FIKP98], queremos construir um conjunto de caminhos tal que a principal contribuição para o termo (3.1.7) venha do somatório. No que segue, apresentaremos algumas motivações para a construção e como construímos o conjunto completo de caminhos $\Gamma$.

\subsubsection{Motivações e Escolha do Conjunto de Caminhos}

Em um breve resumo, queremos construir um conjunto de caminhos auto-evitantes que seja completo, que o caminho mais longo tenha comprimento no máximo $V$ e que todos os caminhos desse conjunto não passem por sítios com alta energia (definiremos o que significa isso mais adiante). Esta última propriedade requerida é para garantir que o fator $e^{\beta\left[H(\boldsymbol{\sigma}) \vee H\left(\boldsymbol{\sigma}^{\prime}\right)\right]}$ dentro do sinal de max em (3.1.7) contribua apenas como um termo de erro para K. Na sequência, apresentaremos algumas razões que nos motivam a construir um conjunto satisfazendo essas propriedades.

Vamos supor inicialmente que o termo $H\left(\boldsymbol{\sigma}^{\prime}\right) \vee H(\boldsymbol{\sigma})$ dentro do sinal de max em (3.1.7) é tal que, para todo $\rho>0$ e todo elo $e=\left(\boldsymbol{\sigma}, \boldsymbol{\sigma}^{\prime}\right) \in \mathcal{E}$,

$$
H\left(\boldsymbol{\sigma}^{\prime}\right) \vee H(\boldsymbol{\sigma}) \leq \rho N
$$


Esta última condição indica que $e^{\beta\left[H\left(\boldsymbol{\sigma}^{\prime}\right) \vee H(\boldsymbol{\sigma})\right]}$ contribui apenas como um termo de erro para (3.1.7). Neste caso, temos que

$$
\mathrm{K} \leq \frac{V e^{\beta \rho N}}{Z} \max _{e=\left(\boldsymbol{\sigma}, \boldsymbol{\sigma}^{\prime}\right)} \sum_{\gamma_{\boldsymbol{\eta}, \boldsymbol{\eta}^{\prime}} \ni e}\left|\gamma_{\boldsymbol{\eta}, \boldsymbol{\eta}^{\prime}}\right| e^{-\beta\left[H(\boldsymbol{\eta})+H\left(\boldsymbol{\eta}^{\prime}\right)\right]} .
$$

Agora, se escolhermos um conjunto de caminhos $\Gamma$ satisfazendo, em primeiro lugar, a condição,

$$
\forall \gamma \in \Gamma, \quad|\gamma| \leq V
$$

então poderíamos ignorar a contribuição dada por $\left|\gamma_{\boldsymbol{\eta}, \boldsymbol{\eta}^{\prime}}\right|$ em (3.2.2), já que poderíamos sempre cotá-la por $V$ e, como $\log V=o(N)$, isto implicaria lidar apenas com

$$
\max _{e \in \mathcal{E}} \sum_{\gamma_{\boldsymbol{\eta}, \boldsymbol{\eta}^{\prime}} \ni e} e^{-\beta\left[H(\boldsymbol{\eta})+H\left(\boldsymbol{\eta}^{\prime}\right)\right]}
$$

e segundo, para qualquer elo $e=\left(\boldsymbol{\sigma}, \boldsymbol{\sigma}^{\prime}\right) \in \mathcal{E}$, a soma

$$
\sum_{\gamma_{\boldsymbol{\eta}, \boldsymbol{\eta}^{\prime}} \ni e} e^{-\beta\left[H(\boldsymbol{\eta})+H\left(\boldsymbol{\eta}^{\prime}\right)\right]}
$$

fatore em um produto de duas somas as quais possam ser vistas como funções de subpartição, em que o prefixo sub significa que são funções de partição de GREM's com as mesmas famílias de parâmetros que o GREM inicial, isto é, $\left\{p_{j}\right\}_{j=1}^{n}$ e $\left\{a_{j}\right\}_{j=1}^{n}$, mas com volumes que são frações de $V$. Um argumento para estimar essas duas somas seguiria de uma combinação das estratégias usadas em [FIKP98] e [CCP87], com as devidas adaptações.

Observação 3.2.1. Observe que, qualquer que seja o conjunto completo de caminhos $\Gamma$ que consideremos em (3.2.2), se ele satisfaz a condição (3.2.3), podemos facilmente obter uma cota superior para $\tau_{N}$ se impormos, adicionalmente, um regime de temperaturas baixas para o sistema, isto é, se $\beta \geq \beta_{\ell_{n}}$. De fato, não é difícil ver que, qualquer que seja o elo $e=\left(\boldsymbol{\sigma}, \boldsymbol{\sigma}^{\prime}\right) \in \mathcal{E}$,

$$
\sum_{\gamma_{\boldsymbol{\eta}, \boldsymbol{\eta}^{\prime}} \ni e}\left|\gamma_{\boldsymbol{\eta}, \boldsymbol{\eta}^{\prime}}\right| e^{-\beta\left[H(\boldsymbol{\eta})+H\left(\boldsymbol{\eta}^{\prime}\right)\right]} \leq V\left(\sum_{\boldsymbol{\eta} \in \Omega_{\mathcal{V}}} e^{-\beta H(\boldsymbol{\eta})}\right)\left(\sum_{\boldsymbol{\eta}^{\prime} \in \Omega_{\mathcal{V}}} e^{-\beta H\left(\boldsymbol{\eta}^{\prime}\right)}\right)=V Z^{2}
$$

e portanto, de (3.2.2),

$$
\mathrm{K} \leq V^{2} e^{\rho \beta N} Z
$$

Como consequência, para todo $\beta>0$, de (3.1.5) e do Corolário 2.1.1, com $\mathbb{P}$-probabilidade 1 ,

$$
\limsup _{N \uparrow \infty} \frac{1}{N} \log \tau_{N} \leq \limsup _{N \uparrow \infty} \frac{1}{N} \log \mathrm{K} \leq F_{n}(\beta)+\rho \beta .
$$

Usando agora o fato que a desigualdade acima vale para todo $\rho>0$, se $\beta \geq \beta_{\ell_{n}}$, então, combinando a desigualdade acima com (2.1.56), temos que

$$
\limsup _{N \uparrow \infty} \frac{1}{N} \log \tau_{N} \leq \beta \sum_{\ell=1}^{\ell_{n}} \beta_{\ell} \sum_{k=J_{\ell-1}^{*}+1}^{J_{\ell}^{*}} a_{k}
$$


e o Teorema 2.3.1 fica provado para o regime de temperaturas baixas. Voltando em (3.2.8), observe ainda que a cota obtida é válida para todo $\beta>0$. A questão neste caso é que, quando mudamos de regime de temperatura, de baixas $\left(\beta \geq \beta_{\ell_{n}}\right)$ para intermediárias $\left(\beta_{\ell} \leq \beta<\beta_{\ell+1}\right)_{\ell=1}^{\ell_{n}-1}$ ou altas temperaturas $\left(\beta<\beta_{1}\right), F_{n}(\beta)$ muda de uma função linear para uma função quadrática de $\beta$ (veja (2.1.56)) que, em geral, é maior que a linear. De fato, se $\beta_{\ell} \leq \beta<\beta_{\ell+1}$, para algum $\ell \in\left\{0, \ldots, \ell_{n}-1\right\}$, então,

$$
\begin{aligned}
F_{n}(\beta)-\beta & \sum_{i=1}^{\ell_{n}} \beta_{i} \sum_{k=J_{i-1}^{*}+1}^{J_{i}^{*}} a_{k} \\
& \stackrel{(2.1 .56)}{=} \beta \sum_{i=1}^{\ell} \beta_{i} \sum_{k=J_{i-1}^{*}+1}^{J_{i}^{*}} a_{k}+\frac{1}{2} \sum_{k=J_{\ell}^{*}+1}^{n}\left(\beta_{\star}^{2} p_{k}+\beta^{2} a_{k}\right)-\beta \sum_{i=1}^{\ell_{n}} \beta_{i} \sum_{k=J_{i-1}^{*}+1}^{J_{i}^{*}} a_{k} \\
& \stackrel{(2.1 .52)}{=} \frac{1}{2} \sum_{i=\ell+1}^{\ell_{n}} \sum_{k=J_{i-1}^{*}+1}^{J_{i}^{*}}\left(\beta_{i}^{2}-2 \beta \beta_{i}+\beta^{2}\right) a_{k} \\
= & \frac{1}{2} \sum_{i=\ell+1}^{\ell_{n}} \sum_{k=J_{i-1}^{*}+1}^{J_{i}^{*}}\left(\beta_{i}-\beta\right)^{2} a_{k}>0 .
\end{aligned}
$$

O problema aqui é que não podemos realmente excluir a possibilidade de que $H(\boldsymbol{\sigma}) \vee H\left(\boldsymbol{\sigma}^{\prime}\right)$ seja positivo e grande, pois não há como evitar, sempre, que os caminhos visitem sítios com alta energia. Isto segue porque $\Gamma$ é um conjunto completo de caminhos. Entretanto, esta situação pode ser contornada. Uma abordagem geral para isso consiste em construir, para cada par de sítios distintos $\boldsymbol{\eta}$ e $\boldsymbol{\eta}^{\prime}$ de $\Omega_{\mathcal{V}}$, uma família de caminhos suficientemente grande de tal forma que asseguramos, com alta probabilidade, a existência de, ao menos, um caminho indo de $\boldsymbol{\eta}$ para $\boldsymbol{\eta}^{\prime}$ que evita passar por sítios de alta energia. Neste caso, sítios com alta energia aparecem somente nos extremos dos caminhos, implicando que suas contribuições para o termo do lado direito de (3.1.7) sejam canceladas com algum dos fatores de $e^{-\beta H(\boldsymbol{\eta})}$ ou $e^{-\beta H\left(\boldsymbol{\eta}^{\prime}\right)}$ que aparece dentro do somatório. Escolhendo estes caminhos para o conjunto $\Gamma$, exatamente um para cada par $\boldsymbol{\eta}$ para $\boldsymbol{\eta}^{\prime}$, obtemos um conjunto completo de caminhos tais que, com alta probabilidade, o fator $e^{\beta\left[H(\boldsymbol{\sigma}) \vee H\left(\boldsymbol{\sigma}^{\prime}\right)\right]}$ contribui apenas como termo de erro. Começaremos a construção do conjunto $\Gamma$ a seguir.

Iniciaremos a construção da família de caminhos $\Gamma$ relembrando, veja (2.1.3) e (2.1.19), que o conjunto de partículas $\mathcal{V}$ de volume $V$ pode ser decomposto como a união de $n$ subconjuntos $\mathcal{V}_{j}$ de volume $V_{j}$ cada um podendo ser escrito como a união de $\mathrm{B}$ blocos $\mathcal{V}_{j}(i)$ de cardinalidade $\mathrm{v}_{j}$, ou seja,

$$
\mathcal{V}=\bigcup_{j=1}^{n} \mathcal{V}_{j}=\bigcup_{j=1}^{n} \bigcup_{i=1}^{\mathrm{B}} \mathcal{V}_{j}(i)
$$


Dada uma configuração $\boldsymbol{\sigma}=\sigma_{1}, \ldots, \sigma_{n} \in \Omega \mathcal{V}$, a decomposição acima induz às configurações $\sigma_{j} \in \Omega \mathcal{V}_{j}$ a seguinte decomposição,

$$
\sigma_{j} \stackrel{\text { def }}{=} \sigma_{j, 1}, \sigma_{j, 2}, \ldots, \sigma_{j, \mathrm{~B}}
$$

em que para cada $j=1, \ldots, n$ e cada $i=1, \ldots, \mathrm{B}, \sigma_{j, i}=\left(\sigma_{j, i}(\ell)\right)_{\ell \in \mathcal{V}_{j}(i)}$ em que $\sigma_{j, i}: \mathcal{V}_{j}(i) \rightarrow$ $\{-1,+1\}$. O ponto chave desta decomposição é que há o mesmo número de blocos, $B$, em todos os conjuntos $\mathcal{V}_{j}$, mudando apenas a cardinalidade dos blocos que depende do nível considerado. Essa "igualdade" entre os níveis será fundamental para conseguirmos escrever (3.2.5) como produto de duas funções de subpartição e também simplificar nossas estimativas.

Dados $\boldsymbol{\eta} \neq \boldsymbol{\eta}^{\prime}$, vamos denotar o conjunto de discrepâncias entre $\boldsymbol{\eta}$ e $\boldsymbol{\eta}^{\prime}$ por $\mathcal{D}_{\mathcal{V}}\left(\boldsymbol{\eta}, \boldsymbol{\eta}^{\prime}\right)$, isto é, o subconjunto de $\mathcal{V}$ em que as duas configurações discordam. Ele pode ser decomposto de acordo com (3.2.11) como

$$
\mathcal{D}_{\mathcal{V}}\left(\boldsymbol{\eta}, \boldsymbol{\eta}^{\prime}\right)=\bigcup_{j=1}^{n} \mathcal{D}_{\mathcal{V}_{j}}\left(\boldsymbol{\eta}, \boldsymbol{\eta}^{\prime}\right)=\bigcup_{j=1}^{n} \bigcup_{i=1}^{\mathrm{B}} \mathcal{D}_{\mathcal{V}_{j}(i)}\left(\boldsymbol{\eta}, \boldsymbol{\eta}^{\prime}\right),
$$

em que $\mathcal{D}_{\mathcal{V}_{j}(i)}\left(\boldsymbol{\eta}, \boldsymbol{\eta}^{\prime}\right) \stackrel{\text { def }}{=} \varnothing$ se não houver discrepâncias em $\mathcal{V}_{j}(i)$

Seja $\Gamma^{1}, \ldots, \Gamma^{\mathrm{v}_{1}}$ uma família de conjuntos de caminhos, tal que

$$
\Gamma^{\ell} \stackrel{\text { def }}{=}\left\{\gamma_{\boldsymbol{\eta}, \boldsymbol{\eta}^{\prime}}^{\ell}: \boldsymbol{\eta}, \boldsymbol{\eta}^{\prime} \in \Omega \mathcal{V}, \boldsymbol{\eta} \neq \boldsymbol{\eta}^{\prime}\right\}, \quad \ell=1, \ldots, \mathrm{v}_{1}
$$

em que os caminhos $\gamma_{\boldsymbol{\eta}, \boldsymbol{\eta}^{\prime}}^{\ell}$ são construídos usando o seguinte algoritmo:

Definição 3.2.1 (Algoritmo de construção do caminho $\gamma_{\boldsymbol{\eta}, \boldsymbol{\eta}^{\prime}}^{\ell}$ ). Para $\ell \in \mathcal{V}_{1}(1)$, seja $\gamma_{\boldsymbol{\eta}, \boldsymbol{\eta}^{\prime}}^{\ell}$ o caminho construído de $\boldsymbol{\eta}$ para $\boldsymbol{\eta}^{\prime}$ trocando-se os spins dos pontos em $\mathcal{D}_{\mathcal{V}}\left(\boldsymbol{\eta}, \boldsymbol{\eta}^{\prime}\right)$ começando pela coordenada $\ell$ de acordo com as regras seguintes.

Passo 1. Considere o bloco $\mathcal{V}_{1}(1)$ e cheque se $\mathcal{D}_{\mathcal{V}_{1}(1)}\left(\boldsymbol{\eta}, \boldsymbol{\eta}^{\prime}\right) \cap\left\{\ell, \ell+1, \ldots, v_{1}\right\}=\varnothing$.

Se sim, então vá para o Passo 2.

Se não, então troque sucessivamente os spins das coordenadas em $\mathcal{D}_{\mathcal{V}_{1}(1)}\left(\boldsymbol{\eta}, \boldsymbol{\eta}^{\prime}\right) \cap\{\ell, \ell+$ $\left.1, \ldots, v_{1}\right\}$. Vá para o Passo 2.

Passo 2. Cheque se $\cup_{j=2}^{n} \mathcal{D}_{\mathcal{V}_{j}(1)}\left(\boldsymbol{\eta}, \boldsymbol{\eta}^{\prime}\right)=\varnothing$.

Se sim, então vá para o Passo 3.

Se não, considere o bloco $\mathcal{V}_{\mathfrak{n}_{1}}(1)$, em que

$$
\mathfrak{n}_{1} \stackrel{\text { def }}{=} \inf \left\{2 \leq j \leq n: \mathcal{D}_{\mathcal{V}_{j}(1)}\left(\boldsymbol{\eta}, \boldsymbol{\eta}^{\prime}\right) \neq \varnothing\right\}
$$


é o primeiro nível $\geq 2$ em que as configurações $\boldsymbol{\eta}$ e $\boldsymbol{\eta}^{\prime}$ discordam no bloco 1 , e troque sucessivamente os spins das coordenadas em $\mathcal{V}_{\mathfrak{n}_{1}}(1)$. Em seguida, vá para o bloco $\mathcal{V}_{\mathfrak{n}_{1}+1}(1)$ e faça o mesmo que foi feito em $\mathcal{V}_{\mathfrak{n}_{1}}(1)$ se isso for possível, então vá para o bloco $\mathcal{V}_{\mathfrak{n}_{1}+2}(1)$ e troque sucessivamente os spins necessários, e continue assim até o último nível, inclusive. Quando $\mathfrak{n}_{1}+k=n$, então todas as discrepâncias em $\cup_{j=2}^{n} \mathcal{D}_{\mathcal{V}_{j}(1)}\left(\boldsymbol{\eta}, \boldsymbol{\eta}^{\prime}\right)$ estão zeradas. Vá para o Passo 3.

Passo 3. Seja

$$
\mathfrak{i}^{*} \stackrel{\text { def }}{=} \inf \left\{2 \leq i \leq \mathrm{B}: \cup_{j=1}^{n} \mathcal{D}_{\mathcal{V}_{j}(i)}\left(\boldsymbol{\eta}, \boldsymbol{\eta}^{\prime}\right) \neq \varnothing\right\}
$$

e

$$
\mathfrak{j}_{i}^{*} \stackrel{\text { def }}{=} \inf \left\{1 \leq j \leq n: \mathcal{D}_{\mathcal{V}_{j}(i)}\left(\boldsymbol{\eta}, \boldsymbol{\eta}^{\prime}\right) \neq \varnothing\right\}, \quad i=2, \ldots, \mathrm{B}
$$

o primeiro nível em que as configurações $\boldsymbol{\eta}$ e $\boldsymbol{\eta}^{\prime}$ discordam exatamente no bloco $i \geq 2$.

Considere então o bloco $\mathcal{V}_{\mathfrak{i}^{*}}\left(\mathfrak{i}^{*}\right)$ e troque sucessivamente todos os spins das coordenadas em $\mathcal{D}_{\mathcal{V}_{\mathfrak{i}^{*}}\left(i^{*}\right)}\left(\boldsymbol{\eta}, \boldsymbol{\eta}^{\prime}\right)$. Em seguida, vá para o bloco $\mathcal{V}_{\mathfrak{i}^{*}+1}\left(\mathfrak{i}^{*}\right)$ e faça o mesmo que foi feito em $\mathcal{V}_{\mathfrak{i}^{*}}\left(\mathfrak{i}^{*}\right)$, caso seja possível. Então, vá para o bloco $\mathcal{V}_{\mathfrak{j}^{*}+2}\left(\mathfrak{i}^{*}\right)$ e troque sucessivamente os spins necessários, e continue assim até o último nível. Quando $\mathfrak{j}_{\mathfrak{i}^{*}}^{*}+k=n$, todas as discrepâncias em $\cup_{j=1}^{n} \mathcal{D}_{\mathcal{V}_{j}\left(i^{*}\right)}\left(\boldsymbol{\eta}, \boldsymbol{\eta}^{\prime}\right)$ estão zeradas.

Agora, considere o bloco $\mathcal{V}_{\mathfrak{i}^{*}+1}^{*}\left(i^{*}+1\right)$ e faça o mesmo que foi feito em $\mathcal{V}_{\mathfrak{i}^{*}}\left(i^{*}\right)$ se isso for possível. Depois, faça o mesmo com $\mathcal{V}_{\mathfrak{i}^{*}+2}\left(\mathfrak{i}^{*}+2\right)$ e continue assim até o último bloco. Quando $\mathfrak{i}^{*}+k=\mathrm{B}$, todas as discrepâncias em $\cup_{j=1}^{n} \cup_{i=2}^{\mathrm{B}} \mathcal{D}_{\mathcal{V}_{j}(i)}\left(\boldsymbol{\eta}, \boldsymbol{\eta}^{\prime}\right)$ estão zeradas. Vá para o Passo 4 .

Passo 4. Volte para o bloco $\mathcal{V}_{1}(1)$ e cheque se $\mathcal{D}_{\mathcal{V}_{1}(1)}\left(\boldsymbol{\eta}, \boldsymbol{\eta}^{\prime}\right) \cap\{1, \ldots, \ell-1\}=\varnothing$.

Se sim, então as discrepâncias em $\mathcal{D}_{\mathcal{V}}\left(\boldsymbol{\eta}, \boldsymbol{\eta}^{\prime}\right)$ foram zeradas e o caminho está construído.

Se não, então troque sucessivamente os spins das coordenadas em $\mathcal{D}_{\mathcal{V}_{1}(1)}\left(\boldsymbol{\eta}, \boldsymbol{\eta}^{\prime}\right) \cap\{1, \ldots, \ell-1\}$ e o caminho está construído.

Observação 3.2.2. Note que, por construção, todos os caminhos em $\bigcup_{\ell=1}^{v_{1}} \Gamma^{\ell}$ são auto-evitantes e tem comprimento no máximo $V$.

Nossa estratégia aqui será construir $\Gamma$ usando os caminhos do conjunto $\bigcup_{\ell=1}^{v_{1}} \Gamma^{\ell}$. Vale relembrar então que devemos escolher para $\Gamma$ exatamente um caminho em $\bigcup_{\ell=1}^{v_{1}} \Gamma^{\ell}$ para cada par de sítios distintos de $\Omega_{\mathcal{V}}$. Além disso, como foi motivado no início desta seção, precisamos levar em conta para essas escolhas o fato que os caminhos devem evitar configurações de alta energia. Para tanto, devemos primeiro introduzir o que venha ser uma configuração de alta energia. 
Definição 3.2.2 (Interior de um Caminho). Dados $\boldsymbol{\eta}, \boldsymbol{\eta}^{\prime} \in \Omega_{\mathcal{V}}$ e um caminho $\gamma_{\boldsymbol{\eta}, \boldsymbol{\eta}^{\prime}}$ indo de $\boldsymbol{\eta}$ à $\eta^{\prime}$ sobre $\Omega \mathcal{V}$, definimos por

$$
\gamma_{\boldsymbol{\eta}, \boldsymbol{\eta}^{\prime}}^{\mathrm{int}}=\gamma_{\boldsymbol{\eta}, \boldsymbol{\eta}^{\prime}} \backslash\left\{\boldsymbol{\eta}, \boldsymbol{\eta}^{\prime}\right\}
$$

o conjunto dos pontos interiores do caminho $\gamma_{\boldsymbol{\eta}, \boldsymbol{\eta}^{\prime}}$.

Definição 3.2.3 (Sítio, Caminho e Conjunto Bom). Dado $\rho>0$ arbitrário, dizemos que um sítio $\boldsymbol{\sigma}$ é bom (de baixa energia) se $H(\boldsymbol{\sigma}) \leq \rho N$. Se $\boldsymbol{\sigma}$ não é bom, dizemos que ele é ruim (de alta energia). Dizemos que um caminho $\gamma$ é bom se todos os seus pontos interiores são bons e, que um conjunto de caminhos é bom se todos os seus elementos são bons.

Observação 3.2.3. Pela definição acima, note que os caminhos que estamos interessados para $\Gamma$ são os ditos bons. Obviamente, por causa dessa definição, a "bondade" de um caminho é uma propriedade aleatória. Isso significa que o conjunto $\Gamma$ será um conjunto aleatório de caminhos.

Definição 3.2.4. Para cada par de vértices $\boldsymbol{\sigma}, \boldsymbol{\sigma}^{\prime} \in \Omega_{\mathcal{V}}$, definimos a distância de Hamming restrito ao bloco $\mathcal{V}_{1}(1)$ entre $\boldsymbol{\sigma}$ e $\boldsymbol{\sigma}^{\prime}$ por

$$
\mathrm{d}_{1}\left(\boldsymbol{\sigma}, \boldsymbol{\sigma}^{\prime}\right) \stackrel{\text { def }}{=} \frac{1}{2} \sum_{\ell \in \mathcal{V}_{1}(1)}\left|\sigma_{1,1}(\ell)-\sigma_{1,1}^{\prime}(\ell)\right|=\frac{1}{2} \sum_{\ell=1}^{\mathrm{V}_{1}}\left|\sigma_{1,1}(\ell)-\sigma_{1,1}^{\prime}(\ell)\right| .
$$

A coleção de caminhos $\Gamma$ é então construída da seguinte forma.

Definição 3.2.5. Seja $\kappa \in\left(0, \frac{1}{2}\right)$ arbitrário.

(i) Para pares de sítios distintos $\left(\boldsymbol{\eta}, \boldsymbol{\eta}^{\prime}\right)$ tais que $\mathrm{d}_{1}\left(\boldsymbol{\eta}, \boldsymbol{\eta}^{\prime}\right) \geq \kappa \mathrm{v}_{1}$, se existir um caminho bom em $\left\{\gamma_{\boldsymbol{\eta}, \boldsymbol{\eta}^{\prime}}^{\ell}\right\}_{\ell=1}^{v_{1}}$, então escolha o primeiro e coloque-o em $\Gamma$; caso contrário, escolha $\gamma_{\boldsymbol{\eta}, \boldsymbol{\eta}^{\prime}}^{1}$.

(ii) Para pares de sítios distintos $\left(\boldsymbol{\eta}, \boldsymbol{\eta}^{\prime}\right)$ tais que $\mathrm{d}_{1}\left(\boldsymbol{\eta}, \boldsymbol{\eta}^{\prime}\right)<\kappa \mathrm{v}_{1}$, se existir um sítio bom, digamos $\boldsymbol{\omega} \in \Omega_{\mathcal{V}}$, tal que $\mathrm{d}_{1}(\boldsymbol{\eta}, \boldsymbol{\omega}) \geq \kappa \mathrm{v}_{1}, \kappa \mathrm{v}_{1} \leq \mathrm{d}_{1}\left(\boldsymbol{\omega}, \boldsymbol{\eta}^{\prime}\right)=\mathrm{d}_{\mathrm{H}}\left(\boldsymbol{\omega}, \boldsymbol{\eta}^{\prime}\right) \leq 2 \kappa \mathrm{v}_{1}$ e existam caminhos bons, um em $\left\{\gamma_{\boldsymbol{\eta}, \boldsymbol{\omega}}^{\ell}\right\}_{\ell=1}^{v_{1}}$ e outro em $\left\{\gamma_{\boldsymbol{\omega}, \boldsymbol{\eta}^{\prime}}^{\ell}\right\}_{\ell=1}^{v_{1}}$ tais que a união destes dois caminhos bons seja um caminho auto-evitante e tenha comprimento no máximo $V$, então tome-o como o caminho conectando $\boldsymbol{\eta}$ e $\boldsymbol{\eta}^{\prime}$ em $\Gamma$; caso contrário, escolha $\gamma_{\boldsymbol{\eta}, \boldsymbol{\eta}^{\prime}}^{1}$.

É evidente que o conjunto $\Gamma$ definido acima é um conjunto completo de caminhos autoevitantes. Além disso, todos os seus elementos têm comprimento no máximo $V \leq N$. Logo, para o conjunto $\Gamma$ dado acima, por (3.1.7), é imediato que

$$
\mathrm{K} \leq \frac{N^{2}}{Z} \max _{e=\left(\boldsymbol{\sigma}, \boldsymbol{\sigma}^{\prime}\right)} e^{\beta\left[H(\boldsymbol{\sigma}) \vee H\left(\boldsymbol{\sigma}^{\prime}\right)\right]} \sum_{\gamma_{\boldsymbol{\eta}, \boldsymbol{\eta}^{\prime}} \ni e} e^{-\beta\left[H(\boldsymbol{\eta})+H\left(\boldsymbol{\eta}^{\prime}\right)\right]} .
$$


O ponto realmente importante da Definição 3.2.5 é que para qualquer $\rho>0$ e qualquer $\kappa \in\left(0, \frac{1}{2}\right)$, para todo $N$ grande suficiente, com uma altíssima probabilidade, o conjunto de caminhos $\Gamma$ é bom. Mais precisamente, temos o seguinte resultado.

Proposição 3.2.1. Para todo $\rho>0$ e todo $\kappa \in\left(0, \frac{1}{2}\right)$, com $\mathbb{P}$-probabilidade 1 , o conjunto $\Gamma$ acima é bom para todo $N$ grande o suficiente.

Demonstração. A ideia para provar esta afirmação é bem simples. Argumentaremos que, para qualquer par arbitrário de sítios distintos $\left(\boldsymbol{\eta}, \boldsymbol{\eta}^{\prime}\right)$ de $\Omega_{\mathcal{V}}$, a probabilidade de não encontrarmos nenhum caminho bom conectando eles tal como descrito na Definição 3.2.5 é menor do que um termo somável $e^{-c(\rho, \kappa) N}$ para alguma constante positiva $c(\rho, \kappa)$ que depende de $\rho$ e $\kappa$, os quais podemos escolher tão pequenos quanto quisermos. O resultado seguirá então do Primeiro Lema de Borel-Cantelli.

Começaremos relembrando que, se $X$ é uma variável aleatória com distribuição $\mathcal{N}(0,1)$, então

$$
\mathbb{P}[X>x]=\frac{1}{\sqrt{2 \pi}} \int_{x}^{\infty} e^{-\frac{x^{2}}{2}} d x \leq \frac{1}{x} e^{-x^{2} / 2} \leq e^{-x^{2} / 2}, \quad \forall x \geq 1
$$

Assim, dado $\rho>0$, não difícil ver que, para todo $\sigma \in \Omega \mathcal{V}$,

$$
\begin{aligned}
\mathbb{P}[\boldsymbol{\sigma} \text { é ruim }]=\mathbb{P}[H(\boldsymbol{\sigma})>\rho N] & =\mathbb{P}\left[\frac{H(\boldsymbol{\sigma})}{\sqrt{V}}>\rho \sqrt{N} \sqrt{\frac{N}{V}}\right] \\
& \leq \mathbb{P}[X>\rho \sqrt{N}] \\
& \leq e^{-\frac{1}{2} \rho^{2} N},
\end{aligned}
$$

para todo $N$ grande o suficiente. Isso implica então que para todo $\gamma \in \Gamma$, se $N$ é grande o suficiente,

$$
\mathbb{P}[\gamma \text { ser ruim }]=\mathbb{P}\left[\exists \boldsymbol{\sigma} \in \gamma^{\mathrm{int}} \text { ruim }\right] \leq \underbrace{\left|\gamma^{\mathrm{int}}\right|}_{\leq N} e^{-\frac{1}{2} \rho^{2} N} \leq e^{-\frac{1}{4} \rho^{2} N} .
$$

A partir desse ponto, assumiremos que $N$ é grande o suficiente de tal forma que as desigualdades em (3.2.22) e (3.2.23) são verdade.

i. Considere primeiro os pares de sítios distintos $\left(\boldsymbol{\eta}, \boldsymbol{\eta}^{\prime}\right)$ tais que $\mathrm{d}_{1}\left(\boldsymbol{\eta}, \boldsymbol{\eta}^{\prime}\right) \geq \kappa \mathrm{v}_{1}$. Para obtermos uma cota para a probabilidade de não encontrarmos nenhum caminho bom conectando esses sítios exponencialmente pequena, vamos precisar introduzir aqui um novo conceito, a noção de caminhos independentes. 
Definição 3.2.6 (Caminhos Independentes). Sejam $\gamma_{1}, \ldots, \gamma_{k}$ uma família de caminhos sobre $\Omega_{\mathcal{V}}$. Dizemos que esses caminhos são independentes se, quaisquer que sejam $\boldsymbol{\sigma}^{1} \in \gamma_{1}^{\text {int }}, \ldots, \boldsymbol{\sigma}^{k} \in$ $\gamma_{k}^{\text {int }}$, as variáveis aleatórias $H\left(\boldsymbol{\sigma}^{1}\right), \ldots, H\left(\boldsymbol{\sigma}^{k}\right)$ são independentes.

Observação 3.2.4. Da Observação 2.1.4, note que se uma família de caminhos $\gamma_{1}, \ldots, \gamma_{k}$ sobre $\Omega_{\mathcal{V}}$ é tal que para todo $\boldsymbol{\sigma}^{1} \in \gamma_{1}^{\text {int }}, \ldots, \boldsymbol{\sigma}^{k} \in \gamma_{k}^{\text {int }}$,

$$
\sigma_{1}^{1} \neq \sigma_{1}^{2} \neq \cdots \neq \sigma_{1}^{k}
$$

isto é, o nível 1 de $\boldsymbol{\sigma}^{1}, \boldsymbol{\sigma}^{2}, \ldots, \boldsymbol{\sigma}^{k}$ são todos diferentes um do outro, então essa é uma família de caminhos independentes.

Essa última observação motiva as próximas definições.

Definição 3.2.7 (Caminhos 1-interior-disjunto). Seja $\boldsymbol{\eta}, \boldsymbol{\eta}^{\prime} \in \Omega_{\mathcal{V}}$ sítios distintos e seja $\gamma_{\boldsymbol{\eta}, \boldsymbol{\eta}^{\prime}}$ um caminho indo de $\boldsymbol{\eta}$ à $\boldsymbol{\eta}^{\prime}$. Definimos por

$$
\gamma_{\boldsymbol{\eta}, \boldsymbol{\eta}^{\prime}}^{1 \text { int }}=\left\{\sigma_{1}=\left.\boldsymbol{\sigma}\right|_{\mathcal{V}_{1}}: \boldsymbol{\sigma} \in \gamma_{\boldsymbol{\eta}, \boldsymbol{\eta}^{\prime}}^{\mathrm{int}}\right\}
$$

o conjunto das configurações do nível 1 dos pontos interiores do caminho $\gamma_{\boldsymbol{\eta}, \boldsymbol{\eta}^{\prime}}$. Diremos que uma família de caminhos de $\boldsymbol{\eta}$ para $\boldsymbol{\eta}^{\prime}$ sobre $\Omega_{\mathcal{V}}$, digamos $\gamma_{1}, \gamma_{2}, \ldots, \gamma_{k}$, é 1-interior-disjunto se $\gamma_{\ell}^{1-\text { int }} \cap \gamma_{m}^{1 \text {-int }}=\varnothing$ para todo $1 \leq \ell \neq m \leq k$.

No resultado seguinte, Lema 3.2.1, nós exibimos uma condição suficiente para a existência de caminhos independentes entre pares de sítios de $\Omega_{\mathcal{V}}$.

Lema 3.2.1. Sejam $\boldsymbol{\eta}, \boldsymbol{\eta}^{\prime} \in \Omega_{\mathcal{V}}$. Se $\mathrm{d}_{1}\left(\boldsymbol{\eta}, \boldsymbol{\eta}^{\prime}\right)=k \geq 1$, então existem $k$ caminhos independentes em $\left\{\gamma_{\boldsymbol{\eta}, \boldsymbol{\eta}^{\prime}}^{\ell}\right\}_{\ell=1}^{\mathrm{v}_{1}}$.

Demonstração. Vamos denotar por $1 \leq \ell_{1}<\cdots<\ell_{k} \leq \mathrm{v}_{1}$ todas as coordenadas em que $\eta_{1,1} \mathrm{e}$ $\eta_{1,1}^{\prime}$ discordam. A ideia é mostrar que os caminhos $\gamma_{\boldsymbol{\eta}, \boldsymbol{\eta}^{\prime}}^{\ell_{1}}, \ldots, \gamma_{\boldsymbol{\eta}, \boldsymbol{\eta}^{\prime}}^{\ell_{k}}$ formam uma família 1-interiordisjunta. Se isso for verdade, a conclusão de independência segue imediatamente da Observação 3.2 .4 .

De fato, sejam $1 \leq i \neq j \leq k$ e suponhamos, sem perda de generalidade, que $i<j$. Considere o caminho $\gamma_{\boldsymbol{\eta}, \boldsymbol{\eta}^{\prime}}^{\ell_{j}}$ e note, da Definição 3.2.1, que os primeiros spins trocados sucessivamente em $\boldsymbol{\eta}$ são $\eta_{1,1}\left(\ell_{j}\right), \ldots, \eta_{1,1}\left(\ell_{k}\right)$ e os últimos são $\eta_{1,1}\left(\ell_{1}\right), \ldots, \eta_{1,1}\left(\ell_{j-1}\right)$. Então, se $\boldsymbol{\sigma}^{\prime} \in \gamma_{\boldsymbol{\eta}, \boldsymbol{\eta}^{\prime}}^{\ell_{j}, \text { int }}$, ele é tal que os spins $\sigma_{1,1}^{\prime}\left(\ell_{1}\right), \ldots, \sigma_{1,1}^{\prime}\left(\ell_{k}\right)$ são iguais a

$$
\begin{gathered}
\eta_{1,1}\left(\ell_{1}\right), \ldots, \eta_{1,1}\left(\ell_{i}\right), \ldots, \eta_{1,1}\left(\ell_{j-1}\right), \eta_{1,1}^{\prime}\left(\ell_{j}\right), \ldots, \eta_{1,1}^{\prime}\left(\ell_{j+m}\right), \eta_{1,1}\left(\ell_{j+m+1}\right), \ldots, \eta_{1,1}\left(\ell_{k}\right) \\
m=0, \ldots, k-j-2
\end{gathered}
$$


$\mathrm{ou}$

$$
\eta_{1,1}\left(\ell_{1}\right), \ldots, \eta_{1,1}\left(\ell_{i}\right), \ldots, \eta_{1,1}\left(\ell_{j-1}\right), \eta_{1,1}^{\prime}\left(\ell_{j}\right), \ldots, \eta_{1,1}^{\prime}\left(\ell_{k}\right)
$$

$\mathrm{ou}$

$$
\begin{gathered}
\eta_{1,1}^{\prime}\left(\ell_{1}\right), \ldots, \eta_{1,1}^{\prime}\left(\ell_{1+m}\right), \eta_{1,1}\left(\ell_{2+m}\right), \ldots, \eta_{1,1}\left(\ell_{i}\right), \ldots, \eta_{1,1}\left(\ell_{j-1}\right), \eta_{1,1}^{\prime}\left(\ell_{j}\right), \ldots, \eta_{1,1}^{\prime}\left(\ell_{k}\right) \\
m=0, \ldots, i-2
\end{gathered}
$$

$\mathrm{ou}$

$$
\begin{gathered}
\eta_{1,1}^{\prime}\left(\ell_{1}\right), \ldots, \eta_{1,1}^{\prime}\left(\ell_{i-1}\right), \ldots, \eta_{1,1}^{\prime}\left(\ell_{i+m}\right), \eta_{1,1}\left(\ell_{i+m+1}\right), \ldots, \eta_{1,1}\left(\ell_{j-1}\right), \eta_{1,1}^{\prime}\left(\ell_{j}\right), \ldots, \eta_{1,1}^{\prime}\left(\ell_{k}\right) \\
m=0, \ldots, j-i-2 .
\end{gathered}
$$

Observação 3.2.5. Nós queremos enfatizar aqui que, por consequência do Passo 4 da Definição 3.2.1, os últimos passos do caminho $\gamma_{\boldsymbol{\eta}, \boldsymbol{\eta}^{\prime}}^{\ell_{j}}$ são dados pela troca dos spins das coordenadas em $\mathcal{D}_{\mathcal{V}_{1}(1)}\left(\boldsymbol{\eta}, \boldsymbol{\eta}^{\prime}\right) \cap\left\{1, \ldots, l_{j}-1\right\}$. Esse detalhe é fundamental para essa demonstração.

Por outro lado, agora considerando o caminho $\gamma_{\boldsymbol{\eta}, \boldsymbol{\eta}^{\prime}}^{\ell_{i}}$, se $\boldsymbol{\sigma} \in \gamma_{\boldsymbol{\eta}, \boldsymbol{\eta}^{\prime}}^{\ell_{i}, \text {-int }}$, então ele é tal que os spins $\sigma_{1,1}\left(\ell_{1}\right), \ldots, \sigma_{1,1}\left(\ell_{k}\right)$ são iguais a

$$
\begin{gathered}
\eta_{1,1}\left(\ell_{1}\right), \ldots, \eta_{1,1}\left(\ell_{i-1}\right), \eta_{1,1}^{\prime}\left(\ell_{i}\right), \ldots, \eta_{1,1}^{\prime}\left(\ell_{i+r}\right), \eta_{1,1}\left(\ell_{i+r+1}\right), \ldots, \eta_{1,1}\left(\ell_{k}\right) \\
r=0, \ldots, k-i-2 ;
\end{gathered}
$$

ou

$$
\eta_{1,1}\left(\ell_{1}\right), \ldots, \eta_{1,1}\left(\ell_{i-1}\right), \eta_{1,1}^{\prime}\left(\ell_{i}\right), \ldots, \eta_{1,1}^{\prime}\left(\ell_{j}\right), \ldots, \eta_{1,1}^{\prime}\left(\ell_{k}\right)
$$

$\mathrm{ou}$

$$
\begin{gathered}
\eta_{1,1}^{\prime}\left(\ell_{1}\right), \ldots, \eta_{1,1}^{\prime}\left(\ell_{1+r}\right), \eta_{1,1}\left(\ell_{2+r}\right), \ldots, \eta_{1,1}\left(\ell_{i-1}\right), \eta_{1,1}^{\prime}\left(\ell_{i}\right), \ldots, \eta_{1,1}^{\prime}\left(\ell_{j}\right), \ldots, \eta_{1,1}^{\prime}\left(\ell_{k}\right) \\
r=0, \ldots, i-3 .
\end{gathered}
$$

Por simples checagem, observe que nas sequências em (3.2.26),(3.2.27) e (3.2.28), o spin $\sigma_{1,1}^{\prime}\left(\ell_{i}\right)=\eta_{1,1}\left(\ell_{i}\right)$ por exemplo, enquanto que nas sequências em (3.2.30),(3.2.31) e (3.2.32), $\sigma_{1,1}\left(\ell_{i}\right)=\eta_{1,1}^{\prime}\left(\ell_{i}\right)$, e nas sequências em $(3.2 .29), \sigma_{1,1}^{\prime}\left(\ell_{i-1}\right)=\eta_{1,1}^{\prime}\left(\ell_{i-1}\right)$ por exemplo, mas em $(3.2 .30),(3.2 .31)$ e $(3.2 .32), \sigma_{1,1}\left(\ell_{i-1}\right)=\eta_{1,1}\left(\ell_{i-1}\right)$. Ou seja, $\sigma_{1} \neq \sigma_{1}^{\prime}$. Agora, como $\boldsymbol{\sigma}$ e $\boldsymbol{\sigma}^{\prime}$ são quaisquer, segue que $\gamma_{\boldsymbol{\eta}, \boldsymbol{\eta}^{\prime}}^{\ell_{i}, 1 \text { int }} \cap \gamma_{\boldsymbol{\eta}, \boldsymbol{\eta}^{\prime}}^{\ell_{j}, \text {-int }}=\varnothing$, o que implica $\gamma_{\boldsymbol{\eta}, \boldsymbol{\eta}^{\prime}}^{\ell_{1}}, \ldots, \gamma_{\boldsymbol{\eta}, \boldsymbol{\eta}^{\prime}}^{\ell_{k}}$ ser uma família 1interior-disjunta, concluindo a prova.

Segue portanto do Lema 3.2.1 que, qualquer que seja $\left(\boldsymbol{\eta}, \boldsymbol{\eta}^{\prime}\right)$ tal que $\mathrm{d}_{1}\left(\boldsymbol{\eta}, \boldsymbol{\eta}^{\prime}\right) \geq \kappa \mathbf{v}_{1}$, existe $W_{\boldsymbol{\eta}, \boldsymbol{\eta}^{\prime}} \subset\left\{\gamma_{\boldsymbol{\eta}, \boldsymbol{\eta}^{\prime}}^{\ell}\right\}_{\ell=1}^{\mathrm{v}_{1}}$ subconjunto de caminhos de $\boldsymbol{\eta}$ para $\boldsymbol{\eta}^{\prime}$ com no mínimo $\kappa \mathrm{v}_{1}$ caminhos 1interior-disjunto (e portanto, independentes) e de comprimento no máximo $N$. Logo, de (3.2.23) 
obtemos que

$$
\mathbb{P}\left[W_{\boldsymbol{\eta}, \boldsymbol{\eta}^{\prime}} \text { ser ruim }\right] \leq(\mathbb{P}[\gamma \text { ser ruim }])^{\kappa \mathrm{v}_{1}} \leq e^{-\frac{1}{4} \kappa \rho^{2} \mathrm{v}_{1} N}
$$

Como o número de tais pares de configurações $\left(\boldsymbol{\eta}, \boldsymbol{\eta}^{\prime}\right)$ não é maior que $4^{N}$, então, quaisquer que sejam $\rho>0$ e $\kappa \in\left(0, \frac{1}{2}\right)$, existe $N_{1}=N_{1}(\rho, \kappa) \in \mathbb{N}$ tal que

$$
\sum_{N \geq N_{1}} \sum_{\left(\boldsymbol{\eta}, \boldsymbol{\eta}^{\prime}\right)} \mathbb{P}\left[\bigcap_{\ell=1}^{\mathrm{v}_{1}}\left\{\gamma_{\boldsymbol{\eta}, \boldsymbol{\eta}^{\prime}}^{\ell} \text { é ruim }\right\}\right] \leq \sum_{N \geq N_{1}} 4^{N} e^{-\frac{1}{4} \kappa \rho^{2} \mathrm{v}_{1} N}<\infty
$$

pois $\mathrm{v}_{1} \uparrow \infty$ quando $N \rightarrow \infty$. Isto mostra que para todo $\rho>0$ e todo $\kappa \in\left(0, \frac{1}{2}\right)$,

$$
\sum_{N \geq 1} \sum_{\left(\boldsymbol{\eta}, \boldsymbol{\eta}^{\prime}\right)} \mathbb{P}\left[\bigcap_{\ell=1}^{v_{1}}\left\{\gamma_{\boldsymbol{\eta}, \boldsymbol{\eta}^{\prime}}^{\ell} \text { é ruim }\right\}\right]<\infty
$$

e a conclusão segue do Primeiro Lema de Borel-Cantelli.

ii. Considere agora os pares de sítios distintos $\left(\boldsymbol{\eta}, \boldsymbol{\eta}^{\prime}\right)$ tais que $\mathrm{d}_{1}\left(\boldsymbol{\eta}, \boldsymbol{\eta}^{\prime}\right)<\kappa \mathbf{v}_{1}$. Seja $\mathcal{D}_{\mathcal{V}_{1}(1)}$ o conjunto de discrepâncias entre $\boldsymbol{\eta}$ e $\boldsymbol{\eta}^{\prime}$ em $\mathcal{V}_{1}(1)$ (cf. (3.2.13)) e considere o conjunto $\Omega_{\mathcal{V}}^{\boldsymbol{\eta}, \boldsymbol{\eta}^{\prime}}$ definido como

$$
\begin{gathered}
\Omega_{\mathcal{V}}^{\boldsymbol{\eta}, \boldsymbol{\eta}^{\prime}} \stackrel{\text { def }}{=}\left\{\boldsymbol{\sigma} \in \Omega_{\mathcal{V}}:\left.\boldsymbol{\sigma}\right|_{\mathcal{D}_{\mathcal{V}_{1}(1)}\left(\boldsymbol{\eta}, \boldsymbol{\eta}^{\prime}\right)}=\left.\boldsymbol{\eta}\right|_{\mathcal{D}_{\mathcal{V}_{1}(1)}\left(\boldsymbol{\eta}, \boldsymbol{\eta}^{\prime}\right)}, \mathrm{d}_{1}(\boldsymbol{\sigma}, \boldsymbol{\eta})=\left\lceil\kappa \mathrm{v}_{1}\right\rceil\right. \\
\text { e } \left.\left.\boldsymbol{\sigma}\right|_{\mathcal{V}_{1}^{c}(1)}=\left.\boldsymbol{\eta}^{\prime}\right|_{\mathcal{V}_{1}^{c}(1)}\right\},
\end{gathered}
$$

em que a condição " $\left.\boldsymbol{\sigma}\right|_{\mathcal{D}_{\mathcal{V}_{1}(1)}\left(\boldsymbol{\eta}, \boldsymbol{\eta}^{\prime}\right)}=\left.\boldsymbol{\eta}\right|_{\mathcal{D}_{\mathcal{V}_{1}(1)}\left(\boldsymbol{\eta}, \boldsymbol{\eta}^{\prime}\right)}$ " não está presente se $\mathcal{D}_{\mathcal{V}_{1}(1)}=\varnothing$. Aqui, $\left.\boldsymbol{\sigma}\right|_{S}=$ $(\boldsymbol{\sigma}(\ell))_{\ell \in S}$ é a restrição da configuração $\boldsymbol{\sigma}$ às coordenadas no conjunto $S$.

Dado $\tilde{\boldsymbol{\omega}} \in \Omega_{\mathcal{V}}^{\boldsymbol{\eta}, \boldsymbol{\eta}^{\prime}}$, seja $\gamma_{\tilde{\boldsymbol{\omega}}, \tilde{\boldsymbol{\omega}}^{\prime}}$ o caminho começando na configuração $\tilde{\boldsymbol{\omega}}$, construído trocandose sucessivamente os spins das coordenadas em $\mathcal{D}_{\mathcal{V}_{1}(1)}\left(\boldsymbol{\eta}, \boldsymbol{\eta}^{\prime}\right)$. Aqui, estamos assumindo que $\gamma_{\tilde{\boldsymbol{\omega}}, \tilde{\boldsymbol{\omega}}^{\prime}}=\varnothing$ quando $\mathcal{D}_{\mathcal{V}_{1}(1)}\left(\boldsymbol{\eta}, \boldsymbol{\eta}^{\prime}\right)=\varnothing$.

Agora, não é difícil ver que

$$
\forall \tilde{\boldsymbol{\omega}} \in \Omega_{\mathcal{V}}^{\boldsymbol{\eta}, \boldsymbol{\eta}^{\prime}}, \quad \kappa \mathrm{v}_{1} \leq \mathrm{d}_{1}\left(\tilde{\boldsymbol{\omega}}, \boldsymbol{\eta}^{\prime}\right)=\mathrm{d}_{\mathrm{H}}\left(\tilde{\boldsymbol{\omega}}, \boldsymbol{\eta}^{\prime}\right) \leq 2 \kappa \mathrm{v}_{1}
$$

Isso significa que os elementos de $\Omega_{\mathcal{V}}^{\eta, \eta^{\prime}}$ são bons candidatos a satisfazer a condição (ii) da Definição 3.2.5.

Então, nosso primeiro passo aqui será provar que existe, com $\mathbb{P}$-probabilidade 1 , ao menos um $\tilde{\boldsymbol{\omega}}$ em $\Omega_{\mathcal{V}}^{\boldsymbol{\eta}, \boldsymbol{\eta}^{\prime}}$ tal que todos os pontos de $\gamma_{\tilde{\boldsymbol{\omega}}, \tilde{\boldsymbol{\omega}}^{\prime}}$ são bons.

Lema 3.2.2. Dado $\kappa \in\left(0, \frac{1}{2}\right)$, para todo par $\left(\boldsymbol{\eta}, \boldsymbol{\eta}^{\prime}\right)$ fixado tal que $\mathrm{d}_{1}\left(\boldsymbol{\eta}, \boldsymbol{\eta}^{\prime}\right)<\kappa \mathrm{v}_{1}$, vale que

$$
\left|\Omega_{\mathcal{V}}^{\eta, \eta^{\prime}}\right| \geq(2 \kappa)^{-\kappa \mathrm{v}_{1}}
$$


Demonstração. Fixado $\left(\boldsymbol{\eta}, \boldsymbol{\eta}^{\prime}\right)$, o número de elementos de $\Omega_{\mathcal{V}}^{\boldsymbol{\eta}, \boldsymbol{\eta}^{\prime}}$ é igual ao número de subconjuntos com $\left\lceil\kappa \mathbf{v}_{1}\right\rceil$ elementos do conjunto $\mathcal{V}_{1}(1) \backslash \mathcal{D}_{\mathcal{V}_{1}(1)}\left(\boldsymbol{\eta}, \boldsymbol{\eta}^{\prime}\right)$. Logo,

$$
\left|\Omega_{\mathcal{V}}^{\boldsymbol{\eta}, \boldsymbol{\eta}^{\prime}}\right|=\left(\begin{array}{c}
\mathrm{v}_{1}-\mathrm{d}_{1}\left(\boldsymbol{\eta}, \boldsymbol{\eta}^{\prime}\right) \\
\left\lceil\kappa \mathrm{v}_{1}\right\rceil
\end{array}\right) \geq\left(\begin{array}{c}
\mathrm{v}_{1}-\left\lfloor\kappa \mathrm{v}_{1}\right\rfloor \\
\left\lceil\kappa \mathrm{v}_{1}\right\rceil
\end{array}\right)
$$

e usando que $\left(\begin{array}{c}m \\ k\end{array}\right) \geq\left(\frac{m}{k}\right)^{k}$ para $m \geq k \geq 1$ e as desigualdades básicas para $\lfloor\cdot\rfloor$ e $\lceil\cdot\rceil$, obtemos

$$
\left(\begin{array}{c}
\mathrm{v}_{1}-\left\lfloor\kappa \mathrm{v}_{1}\right\rfloor \\
\left\lceil\kappa \mathrm{v}_{1}\right\rceil
\end{array}\right) \geq\left(\frac{(1-\kappa) \mathrm{v}_{1}}{\kappa \mathrm{v}_{1}+1}\right)^{\kappa \mathrm{v}_{1}}=\left(\frac{1-\kappa}{\kappa+\frac{1}{\mathrm{v}_{1}}}\right)^{\kappa \mathrm{v}_{1}} .
$$

Agora, como $\mathrm{v}_{1} \uparrow \infty$ quando $N \rightarrow \infty$, dado $\kappa \in\left(0, \frac{1}{2}\right)$, temos que $\frac{1}{\mathrm{v}_{1}} \leq \kappa-2 \kappa^{2}$ para todo $N$ grande o suficiente, então,

$$
\left|\Omega_{\mathcal{V}}^{\eta, \eta^{\prime}}\right| \geq\left(\frac{1-\kappa}{\kappa+\frac{1}{\mathrm{v}_{1}}}\right)^{\kappa \mathrm{v}_{1}} \geq\left(\frac{1-\kappa}{\kappa+\kappa-2 \kappa^{2}}\right)^{\kappa \mathrm{v}_{1}}=\left(\frac{1}{2 \kappa}\right)^{\kappa \mathrm{v}_{1}}
$$

Logo, pelo lema acima, podemos construir ao menos $(2 \kappa)^{-\kappa v_{1}}$ daqueles caminhos $\gamma_{\tilde{\boldsymbol{\omega}}, \tilde{\boldsymbol{\omega}}^{\prime}}$, apenas variando o ponto $\tilde{\boldsymbol{\omega}}$. Não difícil ver que eles formam um conjunto 1-interior-disjunto. Então, assumindo que $N$ é grande o suficiente para que a probabilidade do caminho $\gamma_{\tilde{\boldsymbol{\omega}}, \tilde{\boldsymbol{\omega}}^{\prime}}\left(\tilde{\boldsymbol{\omega}}\right.$ e $\tilde{\boldsymbol{\omega}}^{\prime}$ inclusive) não ser bom ser no máximo $e^{-\frac{1}{4} \rho^{2} N}$, como o número de pares de configurações $\left(\boldsymbol{\eta}, \boldsymbol{\eta}^{\prime}\right)$ não é maior que $4^{N}$, dados $\rho>0$ e $\kappa \in\left(0, \frac{1}{2}\right)$, existe $N_{2}=N_{2}(\rho, \kappa) \in \mathbb{N}$ tal que

$$
\sum_{N \geq N_{2}} \sum_{\left(\boldsymbol{\eta}, \boldsymbol{\eta}^{\prime}\right)} \mathbb{P}\left[\bigcap_{\left\{\gamma_{\tilde{\boldsymbol{\omega}}, \tilde{\boldsymbol{\omega}}^{\prime}}: \tilde{\boldsymbol{\omega}} \in \Omega_{\mathcal{V}}^{\eta, \eta^{\prime}}\right\}}\left\{\gamma_{\tilde{\boldsymbol{\omega}}, \tilde{\boldsymbol{\omega}}^{\prime}} \text { é ruim }\right\}\right] \leq \sum_{N \geq N_{2}} 4^{N} e^{-\frac{1}{4} \rho^{2} N(2 \kappa)^{-\kappa v_{1}}}<\infty
$$

pois $(2 \kappa)^{-\kappa v_{1}} \uparrow \infty$ quando $N \rightarrow \infty$, o que implica a convergência da série completa. Logo, do Primeiro Lema de Borel-Cantelli, quaisquer que sejam $\rho>0$ e $\kappa \in\left(0, \frac{1}{2}\right)$, com $\mathbb{P}$-probabilidade 1 , existe ao menos um sítio $\tilde{\boldsymbol{\omega}} \in \Omega_{\mathcal{V}}^{\boldsymbol{\eta}, \boldsymbol{\eta}^{\prime}}$, digamos $\boldsymbol{\omega}$, e o correspondente caminho, digamos $\gamma_{\boldsymbol{\omega}, \boldsymbol{\omega}^{\prime}}$, com todos os seus pontos bons. Por construção, $\boldsymbol{\omega}^{\prime}$ coincide com $\boldsymbol{\eta}^{\prime}$ em $\mathcal{D}_{\mathcal{V}_{1}(1)}\left(\boldsymbol{\eta}, \boldsymbol{\eta}^{\prime}\right)$ e é tal que $\mathrm{d}_{1}\left(\boldsymbol{\omega}^{\prime}, \boldsymbol{\eta}^{\prime}\right) \geq \kappa \mathbf{v}_{1}$. Agora, note que os pares $(\boldsymbol{\eta}, \boldsymbol{\omega})$ e $\left(\boldsymbol{\omega}^{\prime}, \boldsymbol{\eta}^{\prime}\right)$ satisfazem as hipóteses do item anterior. Logo, dados $\rho>0$ e $\kappa \in\left(0, \frac{1}{2}\right)$, com $\mathbb{P}$-probabilidade 1 , existem os caminhos $\gamma_{\boldsymbol{\eta}, \boldsymbol{\omega}}$ e $\gamma_{\boldsymbol{\omega}^{\prime}, \boldsymbol{\eta}^{\prime}}$ bons. Unindo então os três caminhos, $\gamma_{\boldsymbol{\eta}, \boldsymbol{\omega}}, \gamma_{\boldsymbol{\omega}, \boldsymbol{\omega}^{\prime}}$ e $\gamma_{\boldsymbol{\omega}^{\prime}, \boldsymbol{\eta}^{\prime}}$, obtemos um caminho $\gamma_{\boldsymbol{\eta}, \boldsymbol{\eta}^{\prime}}$ que é bom, auto-evitante (a condição " $\left.\boldsymbol{\sigma}\right|_{\mathcal{V}_{1}^{c}(1)}=\left.\boldsymbol{\eta}^{\prime}\right|_{\mathcal{V}_{1}^{c}(1)}$ " em (3.2.36) garante tal propriedade) e tem comprimento no máximo $V$.

Definição 3.2.8. Toda configuração $\boldsymbol{\omega} \in \Omega_{\mathcal{V}}$ como descrito na demonstração acima será chamada de ponto intermediário do caminho $\gamma_{\boldsymbol{\eta}, \boldsymbol{\eta}^{\prime}}$.

Como os casos (i) e (ii) da Definição 3.2.5 foram analisados, a proposição está provada. 


\subsubsection{Primeiras Estimativas}

Seja $\mathcal{G}$ o subconjunto de $\mathcal{E}$ definido por

$$
\mathcal{G} \stackrel{\text { def }}{=}\left\{e=\left(\boldsymbol{\sigma}, \boldsymbol{\sigma}^{\prime}\right) \in \mathcal{E}: e \text { é bom }\right\}
$$

e vamos denotar

$$
\mathrm{K}_{\mathcal{G}} \stackrel{\text { def }}{=} \max _{e=\left(\boldsymbol{\sigma}, \boldsymbol{\sigma}^{\prime}\right) \in \mathcal{G}} e^{\beta\left[H(\boldsymbol{\sigma}) \vee H\left(\boldsymbol{\sigma}^{\prime}\right)\right]} \sum_{\gamma_{\boldsymbol{\eta}, \boldsymbol{\eta}^{\prime}} \ni e} e^{-\beta\left[H(\boldsymbol{\eta})+H\left(\boldsymbol{\eta}^{\prime}\right)\right]}
$$

e

$$
\mathrm{K}_{\mathcal{E} \backslash \mathcal{G}} \stackrel{\text { def }}{=} \max _{e=\left(\boldsymbol{\sigma}, \boldsymbol{\sigma}^{\prime}\right) \in \mathcal{E} \backslash \mathcal{G}} e^{\beta\left[H(\boldsymbol{\sigma}) \vee H\left(\boldsymbol{\sigma}^{\prime}\right)\right]} \sum_{\gamma_{\boldsymbol{\eta}, \boldsymbol{\eta}^{\prime}} \ni e} e^{-\beta\left[H(\boldsymbol{\eta})+H\left(\boldsymbol{\eta}^{\prime}\right)\right]}
$$

Então, reescrevemos o lado direito de (3.2.20) como

$$
\mathrm{K} \leq \frac{N^{2}}{Z}\left(\mathrm{~K}_{\mathcal{G}} \vee \mathrm{K}_{\mathcal{E} \backslash \mathcal{G}}\right)
$$

Estimativa para $L_{\mathcal{E} \backslash \mathcal{G}}$. Pela Proposição 3.2.1, dados $\rho>0$ e $\kappa \in\left(0, \frac{1}{2}\right)$, para quase todas as realizações de $\mathscr{X}$, existe $N_{0}(\rho, \kappa) \in \mathbb{N}$ tal que para todo $N \geq N_{0}$ o conjunto de caminhos $\Gamma$ indicado na Definição 3.2.5 é bom. Isto significa que se existir um vértice ruim em um dado caminho $\gamma$ de $\Gamma$, tal vértice é necessariamente um ponto extremo do caminho, ou seja, dado um caminho $\gamma_{\boldsymbol{\eta}, \boldsymbol{\eta}^{\prime}}$ indo de $\boldsymbol{\eta}$ à $\boldsymbol{\eta}^{\prime}$, se o elo $e=\left(\boldsymbol{\sigma}, \boldsymbol{\sigma}^{\prime}\right) \in \gamma_{\boldsymbol{\eta}, \boldsymbol{\eta}^{\prime}}$ é ruim, então $e$ é o primeiro ou o último elo de $\gamma_{\boldsymbol{\eta}, \boldsymbol{\eta}^{\prime}}$. Neste caso, $\boldsymbol{\sigma}=\boldsymbol{\eta}$ ou $\boldsymbol{\sigma}^{\prime}=\boldsymbol{\eta}^{\prime}$ (note que o caso $\boldsymbol{\sigma}=\boldsymbol{\eta}$ e $\boldsymbol{\sigma}^{\prime}=\boldsymbol{\eta}^{\prime}$ acontece somente se $\left.\gamma_{\boldsymbol{\eta}, \boldsymbol{\eta}^{\prime}}=\left(\boldsymbol{\sigma}, \boldsymbol{\sigma}^{\prime}\right)\right)$. Por simetria é suficiente considerar o primeiro caso. Logo, temos que $e^{\beta\left[H(\boldsymbol{\sigma}) \vee H\left(\boldsymbol{\sigma}^{\prime}\right)\right]}=e^{\beta H(\boldsymbol{\sigma})}$ e $\left\{\left(\boldsymbol{\eta}, \boldsymbol{\eta}^{\prime}\right) \in \Omega_{\mathcal{V}}^{2}: \gamma_{\boldsymbol{\eta}, \boldsymbol{\eta}^{\prime}} \ni e\right\}=\{\boldsymbol{\sigma}\} \times\left(\Omega_{\mathcal{V}} \backslash\{\boldsymbol{\sigma}\}\right)$, e daí,

$$
e^{\beta\left[H(\boldsymbol{\sigma}) \vee H\left(\boldsymbol{\sigma}^{\prime}\right)\right]} \sum_{\gamma_{\boldsymbol{\eta}, \boldsymbol{\eta}^{\prime}} \ni e} e^{-\beta\left[H(\boldsymbol{\eta})+H\left(\boldsymbol{\eta}^{\prime}\right)\right]}=e^{\beta H(\boldsymbol{\sigma})} \sum_{\boldsymbol{\eta} \in \Omega_{\mathcal{V}} \backslash\{\boldsymbol{\sigma}\}} e^{-\beta[H(\boldsymbol{\sigma})+H(\boldsymbol{\eta})]} \leq Z .
$$

Consequentemente,

$$
\mathrm{K}_{\mathcal{E} \backslash \mathcal{G}} \leq Z . \quad \mathbb{P} \text {-q.c. }
$$

Estimativa para $\mathrm{K}_{\mathcal{G}}$. Usando que um sítio bom tem energia no máximo $\rho N$, é imediato que

$$
\mathrm{K}_{\mathcal{G}} \leq e^{\rho \beta N} \max _{e \in \mathcal{G}} \sum_{\gamma_{\boldsymbol{\eta}, \boldsymbol{\eta}^{\prime}} \ni e} e^{-\beta\left[H(\boldsymbol{\eta})+H\left(\boldsymbol{\eta}^{\prime}\right)\right]}
$$

Para estimar este último máximo, a estratégia será usar a definição do conjunto de caminhos $\Gamma$ para dividir a soma $\sum_{\gamma_{\boldsymbol{\eta}, \boldsymbol{\eta}^{\prime}} \ni e}$ em duas: uma restrita aos caminhos $\gamma_{\boldsymbol{\eta}, \boldsymbol{\eta}^{\prime}}$ tais que $\mathrm{d}_{1}\left(\boldsymbol{\eta}, \boldsymbol{\eta}^{\prime}\right) \geq \kappa \mathrm{v}_{1}$ e a outra restrita aos caminhos $\gamma_{\boldsymbol{\eta}, \boldsymbol{\eta}^{\prime}}$ tais que $\mathrm{d}_{1}\left(\boldsymbol{\eta}, \boldsymbol{\eta}^{\prime}\right)<\kappa \mathrm{v}_{1}$. 
Seguindo essa estratégia, de (3.2.49), escrevemos

$$
\mathrm{K}_{\mathcal{G}} \leq 2 e^{\rho \beta N}\left(\mathrm{~K}_{1} \vee \mathrm{K}_{2}\right)
$$

em que

$$
\mathrm{K}_{1} \stackrel{\text { def }}{=} \max _{e \in \mathcal{G}} \sum_{\gamma_{\boldsymbol{\eta}, \boldsymbol{\eta}^{\prime}} \ni e} e^{-\beta\left[H(\boldsymbol{\eta})+H\left(\boldsymbol{\eta}^{\prime}\right)\right]} \mathbb{1}\left\{\mathrm{d}_{1}\left(\boldsymbol{\eta}, \boldsymbol{\eta}^{\prime}\right) \geq \kappa \mathrm{v}_{1}\right\}
$$

e

$$
\mathrm{K}_{2} \stackrel{\text { def }}{=} \max _{e \in \mathcal{G}} \sum_{\gamma_{\boldsymbol{\eta}, \boldsymbol{\eta}^{\prime}} \ni e} e^{-\beta\left[H(\boldsymbol{\eta})+H\left(\boldsymbol{\eta}^{\prime}\right)\right]} \mathbb{1}\left\{\mathrm{d}_{1}\left(\boldsymbol{\eta}, \boldsymbol{\eta}^{\prime}\right)<\kappa \mathrm{v}_{1}\right\}
$$

A estimativa para $\mathrm{K}_{\mathcal{G}}$ agora segue derivando-se cotas para $\mathrm{K}_{1}$ e $\mathrm{K}_{2}$ separadamente. $\mathrm{O}$ argumento para estimar o termo $\mathrm{K}_{1}$ é um pouco longo e o faremos em um capítulo separado, Capítulo 4. A estimativa do termo $\mathrm{K}_{2}$ será derivada no Capítulo 5 onde também concluiremos a prova do Teorema 2.3.1. 


\section{Capítulo 4}

\section{Estimativa Principal}

Neste capítulo derivaremos uma cota para o termo

$$
\mathrm{K}_{1}=\max _{e \in \mathcal{G}} \sum_{\gamma_{\boldsymbol{\eta}, \boldsymbol{\eta}^{\prime}} \ni e} e^{-\beta\left[H(\boldsymbol{\eta})+H\left(\boldsymbol{\eta}^{\prime}\right)\right]} \mathbb{1}\left\{\mathrm{d}_{1}\left(\boldsymbol{\eta}, \boldsymbol{\eta}^{\prime}\right) \geq \kappa \mathbf{v}_{1}\right\}
$$

O capítulo está organizado da seguinte forma. Na Seção 4.1 obteremos algumas estimativas iniciais usando o fato que o conjunto $\Gamma$ está construído utilizando caminhos em $\bigcup_{\ell=1}^{v_{1}} \Gamma^{\ell}$. Ainda como consequência desse fato, obteremos também a fatoração da soma em (4.0.1) como um produto de duas funções de subpartição, o que nos leva a Seção 4.2, onde estimaremos essas duas funções de subpartição usando ideias de [OP84, CCP87, FIKP98, DD02]. Na Seção 4.3 reuniremos as estimativas obtidas nas Seções 4.1 e 4.2 e concluiremos uma cota para $\mathrm{K}_{1}$.

\subsection{Estimativas Preparatórias}

Pela Definição 3.2.5, os caminhos em $\Gamma$ que conectam sítios $\left(\boldsymbol{\eta}, \boldsymbol{\eta}^{\prime}\right)$ tais que $\mathrm{d}_{1}\left(\boldsymbol{\eta}, \boldsymbol{\eta}^{\prime}\right) \geq \kappa \mathrm{v}_{1}$ são escolhidos em $\bigcup_{\ell=1}^{v_{1}} \Gamma^{\ell}$ (exatamente um para cada par). Usando então esse fato, não é difícil ver que

$$
\begin{aligned}
\mathrm{K}_{1} & \leq \max _{e \in \mathcal{G}} \sum_{\gamma_{\boldsymbol{\eta}, \boldsymbol{\eta}^{\prime}} \ni e} e^{-\beta\left[H(\boldsymbol{\eta})+H\left(\boldsymbol{\eta}^{\prime}\right)\right]} \mathbb{1}\left\{\mathrm{d}_{1}\left(\boldsymbol{\eta}, \boldsymbol{\eta}^{\prime}\right) \geq \kappa \mathrm{v}_{1}\right\} \mathbb{1}\left\{\gamma_{\boldsymbol{\eta}, \boldsymbol{\eta}^{\prime}} \in \bigcup_{\ell=1}^{\mathrm{v}_{1}} \Gamma^{\ell}\right\} \\
& \leq \max _{e \in \mathcal{G}} \sum_{\ell=1}^{\mathrm{v}_{1}} \sum_{\gamma_{\boldsymbol{\eta}, \boldsymbol{\eta}^{\prime}} \ni e}^{(\ell)} e^{-\beta\left[H(\boldsymbol{\eta})+H\left(\boldsymbol{\eta}^{\prime}\right)\right]} \underbrace{\mathbb{1}\left\{\mathrm{d}_{1}\left(\boldsymbol{\eta}, \boldsymbol{\eta}^{\prime}\right) \geq \kappa \mathbf{v}_{1}\right\}}_{\leq 1} \\
& \leq \mathrm{v}_{1} \max _{\ell \in \mathcal{I}_{\boldsymbol{v}_{1}}} \max _{e \in \mathcal{G}} \sum_{\gamma_{\boldsymbol{\eta}, \boldsymbol{\eta}^{\prime}} \ni e}^{(\ell)} e^{-\beta\left[H(\boldsymbol{\eta})+H\left(\boldsymbol{\eta}^{\prime}\right)\right]},
\end{aligned}
$$

em que a soma $\sum^{(\ell)}$ está sobre todos os caminhos de $\Gamma^{\ell}$. Observe que substituímos um conjunto aleatório de caminhos por um determinístico.

Para cada $\ell \in \mathcal{I}_{\mathrm{v}_{1}}$, seja $\Lambda_{\ell}$ a variável aleatória definida por

$$
\Lambda_{\ell} \stackrel{\text { def }}{=} \max _{e \in \mathcal{G}} \sum_{\gamma_{\boldsymbol{\eta}, \boldsymbol{\eta}^{\prime}} \ni e}^{(\ell)} e^{-\beta\left[H(\boldsymbol{\eta})+H\left(\boldsymbol{\eta}^{\prime}\right)\right]} .
$$


Note que $\left\{\Lambda_{\ell}\right\}_{\ell=1}^{v_{1}}$ forma uma sequência de variáveis aleatórias identicamente distribuídas sobre $(\mathscr{S}, \Sigma, \mathbb{P})$.

Com a notação acima, escrevemos

$$
\mathrm{K}_{1} \leq \mathrm{v}_{1} \max _{\ell \in \mathcal{I}_{\mathrm{v}_{1}}} \Lambda_{\ell}
$$

O passo seguinte é estimar a variável aleatória $\max _{\ell \in \mathcal{I}_{v_{1}}} \Lambda_{\ell}$. Para isso, é natural considerar apenas a variável $\Lambda_{1}$ porque, como $\left\{\Lambda_{\ell}\right\}_{\ell=1}^{\mathrm{V}_{1}}$ é uma sequência de variáveis identicamente distribuídas,

$$
\mathbb{P}\left[\max _{\ell \in \mathcal{I}_{\mathrm{v}_{1}}} \Lambda_{\ell} \in A\right] \leq \mathrm{v}_{1} \mathbb{P}\left[\Lambda_{1} \in A\right], \quad \forall A \in \Sigma
$$

Se o plano é usar o Primeiro Lema de Borel-Cantelli, então é suficiente achar $A \in \Sigma$, tal que

$$
\mathbb{P}\left[\Lambda_{1} \in A\right] \leq e^{-\varepsilon N}, \quad \forall \varepsilon>0
$$

Considerar a variável $\Lambda_{1}$ quer dizer que o somatório em (4.1.2) está sobre caminhos $\gamma_{\boldsymbol{\eta}, \boldsymbol{\eta}^{\prime}} \in \Gamma^{1}$, em que $\Gamma^{1}=\left\{\gamma_{\boldsymbol{\eta}, \boldsymbol{\eta}^{\prime}}^{1}: \boldsymbol{\eta}, \boldsymbol{\eta}^{\prime} \in \Omega_{\mathcal{V}}\right\}$. Neste caso, dado $e=\left(\boldsymbol{\sigma}, \boldsymbol{\sigma}^{\prime}\right) \in \mathcal{G}$, existe um único $\ell \in \mathcal{I}_{V}$, tal que $\boldsymbol{\sigma}(\ell) \neq \boldsymbol{\sigma}^{\prime}(\ell)$, ou seja, $\boldsymbol{\sigma}^{\prime}$ é a configuração obtida de $\boldsymbol{\sigma}$ trocando-se o spin da coordenada $\ell$. Por causa de (3.2.11), podemos encontrar únicos $j \in \mathcal{I}_{n}$ e $i \in \mathcal{I}_{\mathrm{B}}$ tal que $\ell \in \mathcal{V}_{j}(i)$, e dessa forma, o spin em que $\boldsymbol{\sigma}$ e $\boldsymbol{\sigma}^{\prime}$ discordam fica unicamente determinado por $\sigma_{j, i}(\ell) \neq \sigma_{j, i}(\ell)$. Por construção, o elo $e \in \gamma_{\boldsymbol{\eta}, \boldsymbol{\eta}^{\prime}}$ se o par $\left(\boldsymbol{\eta}, \boldsymbol{\eta}^{\prime}\right)$ é tal que

$$
\eta_{k}= \begin{cases}\eta_{k, 1}, \ldots, \eta_{k, i-1}, \eta_{k, i}, \sigma_{k, i+1}, \ldots, \sigma_{k, \mathrm{~B}}, & \text { se } k=1, \ldots, j \\ \eta_{k, 1}, \ldots, \eta_{k, i-1}, \sigma_{k, i}, \sigma_{k, i+1}, \ldots, \sigma_{k, \mathrm{~B}}, & \text { se } k=j+1, \ldots, n\end{cases}
$$

$\mathrm{e}$

$$
\eta_{k}^{\prime}=\left\{\begin{array}{l}
\sigma_{k, 1}^{\prime}, \ldots, \sigma_{k, i-1}^{\prime}, \sigma_{k, i}^{\prime}, \eta_{k, i+1}^{\prime}, \ldots, \eta_{k, \mathrm{~B}}^{\prime}, \quad \text { se } k=1, \ldots, j \\
\sigma_{k, 1}^{\prime}, \ldots, \sigma_{k, i-1}^{\prime}, \eta_{k, i}^{\prime}, \eta_{k, i+1}^{\prime}, \ldots, \eta_{k, \mathrm{~B}}^{\prime}, \quad \text { se } k=j+1, \ldots, n
\end{array}\right.
$$

em que

$$
\begin{aligned}
\eta_{j, i} & =\eta_{j, i}(\cdot), \ldots, \eta_{j, i}(\ell-1), \sigma_{j, i}(\ell), \sigma_{j, i}(\ell+1), \ldots, \sigma_{j, i}(\cdot) \\
\eta_{j, i}^{\prime} & =\sigma_{j, i}^{\prime}(\cdot), \ldots, \sigma_{j, i}^{\prime}(\ell-1), \sigma_{j, i}^{\prime}(\ell), \eta_{j, i}(\ell+1), \ldots, \eta_{j, i}(\cdot) .
\end{aligned}
$$

Veja a Figura 4.1 para exemplos da descrição acima. Note que os elementos do conjunto $\left\{\left(\boldsymbol{\eta}, \boldsymbol{\eta}^{\prime}\right) \in\right.$ $\left.\Omega_{\mathcal{V}}^{2}: \gamma_{\boldsymbol{\eta}, \boldsymbol{\eta}^{\prime}}^{1} \ni e\right\}$ estão perfeitamente descritos em (4.1.6) e (4.1.7) por meio dos únicos $j \in \mathcal{I}_{n}, i \in$ $\mathcal{I}_{\mathrm{B}}$ e $\ell \in \mathcal{V}_{j}(i)$. A questão é que caracterizar os elementos do conjunto em questão em função de $j, i$ e $\ell$ lida com complicações extras que podem ser evitadas se, por exemplo, substituirmos esse 
conjunto por outro possivelmente maior, mas dependente apenas do bloco onde a discrepância do elo $e$ mora. Isso é possível e ocorre porque todos os níveis da hierarquia podem ser decompostos em uma mesma quantidade de blocos, B, que diverge com $N$ (cf. Observação 2.1.3).

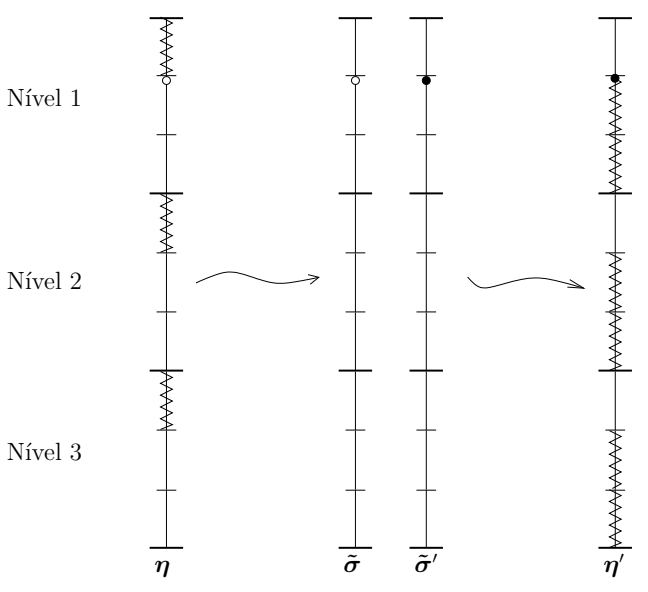

(a)

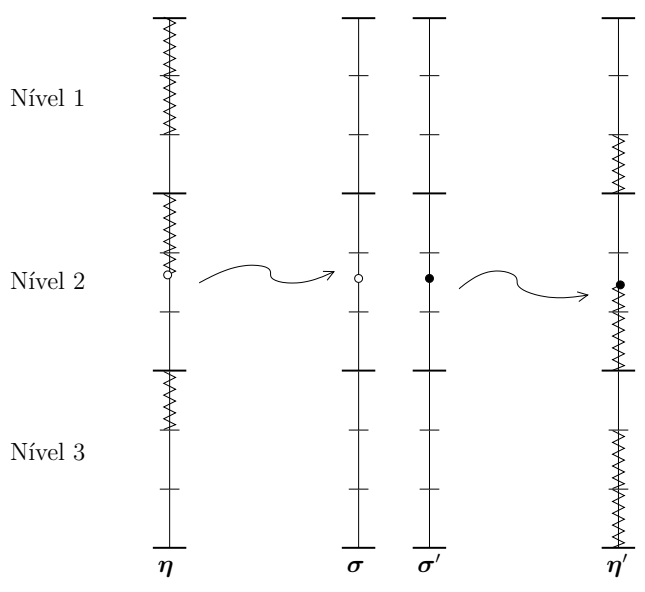

(b)

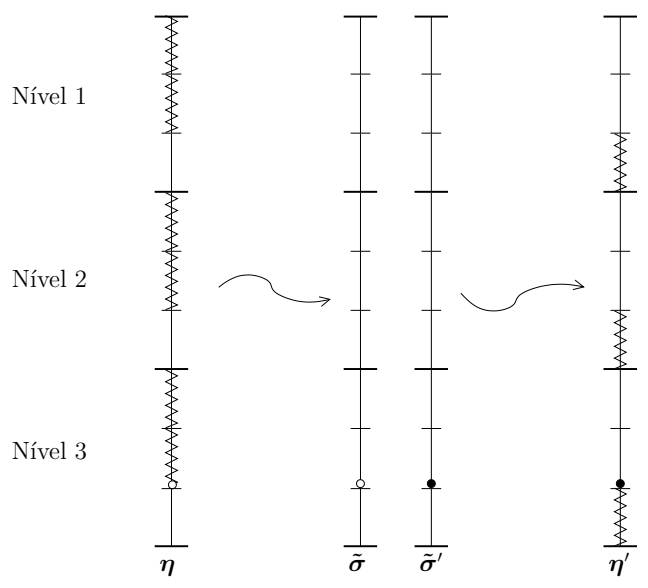

(c)

Figura 4.1: Seja $\Omega_{\mathcal{V}}$ o hipercubo obtido fazendo $\mathrm{B}=n=3$ e $\mathrm{v}_{1}=\mathrm{v}_{2}=\mathrm{v}_{3}$. A figura (a) ilustra os possíveis vértices $\boldsymbol{\eta}$ e $\boldsymbol{\eta}^{\prime}$ em $\Omega_{\mathcal{V}}$ tais que o caminho $\gamma_{\boldsymbol{\eta}, \boldsymbol{\eta}^{\prime}}^{1}$ passa por meio do elo $e=\left(\boldsymbol{\sigma}, \boldsymbol{\sigma}^{\prime}\right)$. O spin de discordância entre $\boldsymbol{\sigma}$ e $\boldsymbol{\sigma}^{\prime}$ está representado por $\circ$ e $\bullet$, respectivamente. Em $\boldsymbol{\eta}$, respectivamente em $\boldsymbol{\eta}^{\prime}$, a linha em zigue-zague representa os spins possivelmente trocados na construção do caminho $\gamma_{\boldsymbol{\eta}, \boldsymbol{\eta}^{\prime}}^{1}$ antes de $\boldsymbol{\sigma}$, respectivamente depois de $\boldsymbol{\sigma}^{\prime}$. As figuras (b) e (c) ilustram o mesmo que (a), mas, agora, para o elo $e^{\prime}=\left(\hat{\boldsymbol{\sigma}}, \hat{\boldsymbol{\sigma}}^{\prime}\right) e^{\prime \prime}=\left(\tilde{\boldsymbol{\sigma}}, \tilde{\boldsymbol{\sigma}}^{\prime}\right)$, respectivamente.

Começaremos com algumas notações e definições adicionais. Dados um bloco $i \in \mathcal{I}_{\mathrm{B}}$ e um 
vértice $\boldsymbol{\sigma} \in \Omega_{\mathcal{V}}$, definimos as coleções de sítios $\Omega_{i}^{-}(\boldsymbol{\sigma})$ e $\Omega_{i}^{+}(\boldsymbol{\sigma})$ por

$$
\Omega_{i}^{-}(\boldsymbol{\sigma}) \stackrel{\text { def }}{=} \begin{cases}\left\{\boldsymbol{\eta} \in \Omega_{\mathcal{V}}: \eta_{j, k}=\sigma_{j, k}, \forall k=i+1, \ldots, \mathrm{B} \text { e } j \in \mathcal{I}_{n}\right\}, & \text { se } i=1, \ldots, \mathrm{B}-1 ; \\ \Omega_{\mathcal{V}}, & \text { se } i=\mathrm{B},\end{cases}
$$

$\mathrm{e}$

$$
\Omega_{i}^{+}(\boldsymbol{\sigma}) \stackrel{\text { def }}{=} \begin{cases}\Omega_{\mathcal{V}}, & \text { se } i=1 ; \\ \left\{\boldsymbol{\eta} \in \Omega_{\mathcal{V}}: \eta_{j, k}=\sigma_{j, k}, \forall k=1, \ldots, i-1 \text { e } j \in \mathcal{I}_{n}\right\}, & \text { se } i=2, \ldots, \mathrm{B} .\end{cases}
$$

Com a notação acima, para um dado elo $e=\left(\boldsymbol{\sigma}, \boldsymbol{\sigma}^{\prime}\right)$, definindo

$$
\hat{\imath}=\hat{\imath}(e) \stackrel{\text { def }}{=}\left\{i \in \mathcal{I}_{\mathrm{B}}: \sigma_{j, i} \neq \sigma_{j, i}^{\prime} \text { para algum } j \in \mathcal{I}_{n}\right\}
$$

isto é, o bloco em que $\boldsymbol{\sigma}$ e $\boldsymbol{\sigma}^{\prime}$ diferem, temos que

$$
\left\{\left(\boldsymbol{\eta}, \boldsymbol{\eta}^{\prime}\right) \in \Omega_{\mathcal{V}}^{2}: \gamma_{\boldsymbol{\eta}, \boldsymbol{\eta}^{\prime}}^{1} \ni e\right\} \subseteq \Omega_{\hat{\imath}}^{-}(\boldsymbol{\sigma}) \times \Omega_{\hat{\imath}}^{+}\left(\boldsymbol{\sigma}^{\prime}\right)
$$

e portanto,

$$
\sum_{\gamma_{\boldsymbol{\eta}, \boldsymbol{\eta}^{\prime}} \ni e}^{(1)} e^{-\beta\left[H(\boldsymbol{\eta})+H\left(\boldsymbol{\eta}^{\prime}\right)\right]} \leq \sum_{\boldsymbol{\eta} \in \Omega_{\hat{\imath}}^{-}(\boldsymbol{\sigma})} e^{-\beta H(\boldsymbol{\eta})} \sum_{\boldsymbol{\eta}^{\prime} \in \Omega_{\hat{\imath}}^{+}\left(\boldsymbol{\sigma}^{\prime}\right)} e^{-\beta H\left(\boldsymbol{\eta}^{\prime}\right)} .
$$

Então,

$$
\begin{aligned}
\Lambda_{1} & =\max _{e \in \mathcal{G}} \sum_{\gamma_{\boldsymbol{\eta}, \boldsymbol{\eta}^{\prime}} \ni e}^{(1)} e^{-\beta\left[H(\boldsymbol{\eta})+H\left(\boldsymbol{\eta}^{\prime}\right)\right]} \\
& \leq \max _{l \in \mathcal{I}_{\mathrm{B}}} \max _{\substack{\boldsymbol{c}\left(\boldsymbol{\sigma}, \boldsymbol{\sigma}^{\prime}\right) \in \mathcal{G} \\
\hat{\imath}(e)=l}} \sum_{\boldsymbol{\eta} \in \Omega_{l}^{-}(\boldsymbol{\sigma})} e^{-\beta H(\boldsymbol{\eta})} \sum_{\boldsymbol{\eta}^{\prime} \in \Omega_{l}^{+}\left(\boldsymbol{\sigma}^{\prime}\right)} e^{-\beta H\left(\boldsymbol{\eta}^{\prime}\right)} .
\end{aligned}
$$

O crucial da desigualdade acima é que as somas do lado direito podem ser vistas como funções de subpartição e, portanto, suas estimativas podem ser feitas com adaptações das técnicas usadas em [CCP87].

Neste ponto, também queremos chamar a atenção do leitor a observar que, para um dado elo $e=\left(\boldsymbol{\sigma}, \boldsymbol{\sigma}^{\prime}\right)$, os elementos de $\Omega_{l}^{-}(\boldsymbol{\sigma})$ e $\Omega_{l}^{+}\left(\boldsymbol{\sigma}^{\prime}\right)$ são combinações de spins conhecidos e desconhecidos. Nosso interesse também é substituir esses conjuntos por outros que caracterizem somente a parte desconhecida das configurações sobre as quais as somas estão em (4.1.14). Para fazer isso, introduzimos a notação seguinte.

Para $1 \leq m \leq k \leq \mathrm{B}$, vamos definir

$$
\mathcal{V}^{m, k} \stackrel{\text { def }}{=} \bigcup_{j=1}^{n} \mathcal{V}_{j}^{m, k}, \operatorname{com} \mathcal{V}_{j}^{m, k} \stackrel{\text { def }}{=} \bigcup_{i=m}^{k} \mathcal{V}_{j}(i)
$$


Naturalmente, também definimos

$$
\Omega_{\mathcal{V}^{m, k}} \stackrel{\text { def }}{=} \Omega_{\mathcal{V}_{1}^{m, k}} \times \cdots \times \Omega_{\mathcal{V}_{n}^{m, k}}, \operatorname{com} \Omega_{\mathcal{V}_{j}^{m, k}}=\{-1,+1\}^{\mathcal{V}_{j}^{m, k}}
$$

Observe que $\mathcal{V}^{1, \mathrm{~B}} \equiv \mathcal{V}$ e, consequentemente, $\Omega_{\mathcal{V}^{1, \mathrm{~B}}} \equiv \Omega_{\mathcal{V}}$

A notação anterior nos permite então escrever os conjuntos $\Omega_{l}^{-}(\boldsymbol{\sigma})$ e $\Omega_{l}^{+}\left(\boldsymbol{\sigma}^{\prime}\right)$ da seguinte forma:

$$
\Omega_{l}^{-}(\boldsymbol{\sigma})= \begin{cases}\left(\Omega_{\mathcal{V}_{1}^{1, l}} \times\left\{\left.\boldsymbol{\sigma}\right|_{\left.\mathcal{V}_{1}^{l+1, \mathrm{~B}}\right\}}\right) \times \cdots \times\left(\Omega_{\mathcal{V}_{n}^{1, l}} \times\left\{\left.\boldsymbol{\sigma}\right|_{\mathcal{V}_{n}^{l+1, \mathrm{~B}}}\right\}\right),\right. & \text { se } l=1, \ldots, \mathrm{B}-1 ; \\ \Omega_{\mathcal{V},} & \text { se } l=\mathrm{B} ;\end{cases}
$$

e

$$
\Omega_{l}^{+}\left(\boldsymbol{\sigma}^{\prime}\right)= \begin{cases}\Omega_{\mathcal{V}}, & \text { se } l=1 ; \\ \left(\left\{\left.\boldsymbol{\sigma}^{\prime}\right|_{\mathcal{V}_{1}^{1, l-1}}\right\} \times \Omega_{\mathcal{V}_{1}^{l, \mathrm{~B}}}\right) \times \cdots \times\left(\left\{\left.\boldsymbol{\sigma}^{\prime}\right|_{\mathcal{V}_{n}^{1, l-1}}\right\} \times \Omega_{\mathcal{V}_{n}^{l, \mathrm{~B}}}\right), & \text { se } l=2, \ldots, \mathrm{B} .\end{cases}
$$

Nosso próximo passo é proceder a estimativa de (4.1.14) de duas formas diferentes: uma considerando $l \in\{1, \mathrm{~B}\}$ e a outra considerando $l \in\{2, \ldots, \mathrm{B}-1\}$.

Começaremos considerando a forma mais simples, quando $l \in\{1, \mathrm{~B}\}$. Neste caso, note que, $\mathrm{ou}$

$$
\Omega_{1}^{-}(\boldsymbol{\sigma})=\left(\Omega_{\mathcal{V}_{1}^{1,1}} \times\left\{\left.\boldsymbol{\sigma}\right|_{\mathcal{V}_{1}^{2, \mathrm{~B}}}\right\}\right) \times \cdots \times\left(\Omega_{\mathcal{V}_{n}^{1,1}} \times\left\{\left.\boldsymbol{\sigma}\right|_{\mathcal{V}_{n}^{2, \mathrm{~B}}}\right\}\right) \quad \text { e } \quad \Omega_{1}^{+}\left(\boldsymbol{\sigma}^{\prime}\right)=\Omega_{\mathcal{V}}
$$

$\mathrm{ou}$

$$
\Omega_{\mathrm{B}}^{-}(\boldsymbol{\sigma})=\Omega_{\mathcal{V}} \quad \text { e } \quad \Omega_{\mathrm{B}}^{+}\left(\boldsymbol{\sigma}^{\prime}\right)=\left(\left\{\left.\boldsymbol{\sigma}^{\prime}\right|_{\mathcal{V}_{1}^{1, \mathrm{~B}-1}}\right\} \times \Omega_{\mathcal{V}_{1}^{\mathrm{B}, \mathrm{B}}}\right) \times \cdots \times\left(\left\{\left.\boldsymbol{\sigma}^{\prime}\right|_{\mathcal{V}_{n}^{1, \mathrm{~B}-1}}\right\} \times \Omega_{\mathcal{V}_{n}^{\mathrm{B}, \mathrm{B}}}\right)
$$

Como $\left|\mathcal{V}_{j}(i)\right|=\mathrm{v}_{j}$ para todo $j \in \mathcal{I}_{n}$ e $i \in \mathcal{I}_{\mathrm{B}}$, temos que

$$
\left|\Omega_{\mathcal{V}_{j}^{k, k}}\right| \stackrel{(4.1 .15)}{=} 2^{\mathrm{v}_{j}} \stackrel{(2.1 .13)}{\leq} 2^{p_{j} \mathcal{S}}
$$

para todo $k \in \mathcal{I}_{\mathrm{B}}$.

Consequentemente, de (4.1.19), (4.1.20) e (4.1.21), qualquer que seja $l \in\{1, B\}$, obtemos que

$$
\sum_{\boldsymbol{\eta} \in \Omega_{l}^{-}(\boldsymbol{\sigma})} e^{-\beta H(\boldsymbol{\eta})} \sum_{\boldsymbol{\eta}^{\prime} \in \Omega_{l}^{+}\left(\boldsymbol{\sigma}^{\prime}\right)} e^{-\beta H\left(\boldsymbol{\eta}^{\prime}\right)} \leq 2^{\mathcal{S} \sum_{j=1}^{n} p_{j}} e^{-\beta H(\overline{\boldsymbol{\sigma}})} Z=2^{\mathcal{S}} e^{-\beta H(\overline{\boldsymbol{\sigma}})} Z,
$$

em que usamos também que $\sum_{j=1}^{n} p_{j}=1$ e estamos denotando por $\overline{\boldsymbol{\sigma}}$ a (única) configuração de $\Omega \mathcal{V}$ tal que

$$
H(\overline{\boldsymbol{\sigma}})=\min _{\boldsymbol{\sigma} \in \Omega_{\mathcal{V}}} H(\boldsymbol{\sigma})
$$


Logo, é imediato que

$$
\max _{l \in\{1, \mathrm{~B}\}} \max _{\substack{\boldsymbol{c}=\left(\boldsymbol{\sigma}, \boldsymbol{\sigma}^{\prime}\right) \in \mathcal{G} \\ \hat{\imath}(e)=l}} \sum_{\boldsymbol{\eta} \in \Omega_{l}^{-}(\boldsymbol{\sigma})} e^{-\beta H(\boldsymbol{\eta})} \sum_{\boldsymbol{\eta}^{\prime} \in \Omega_{l}^{+}\left(\boldsymbol{\sigma}^{\prime}\right)} e^{-\beta H\left(\boldsymbol{\eta}^{\prime}\right)} \leq 2^{\mathcal{S}} e^{-\beta H(\overline{\boldsymbol{\sigma}})} Z .
$$

Usando agora o Teorema 1.5(iii)[BK04a] e o Corolário 2.1.1, para todo $\beta>0$ e todo $\varepsilon>0$, com $\mathbb{P}$-probabilidade 1 ,

$$
\frac{1}{N} \log \left(2^{\mathcal{S}} e^{-\beta H(\overline{\boldsymbol{\sigma}})} Z\right) \leq F_{n}(\beta)+\beta \sum_{i=1}^{\ell_{n}} \beta_{i} \sum_{k=J_{i-1}^{*}+1}^{J_{i}^{*}} a_{k}+\varepsilon,
$$

para todo $N$ grande o suficiente, em que usamos também que $\mathcal{S}=o(N)$.

Vamos considerar agora o caso em que $l \in\{2, \ldots, \mathrm{B}-1\}$. Neste caso, o argumento é um pouco mais longo e se estenderá pelo resto desta seção e pela seção seguinte. Começaremos observando que podemos escrever, para um dado elo $e=\left(\boldsymbol{\sigma}, \boldsymbol{\sigma}^{\prime}\right)$,

$$
\Omega_{l}^{-}(\boldsymbol{\sigma})=\left(\Omega_{\mathcal{V}_{1}^{1, l-1}} \times \Omega_{\mathcal{V}_{1}^{l, l}} \times\left\{\left.\boldsymbol{\sigma}\right|_{\mathcal{V}_{1}^{l+1, \mathrm{~B}}}\right\}\right) \times \cdots \times\left(\Omega_{\mathcal{V}_{n}^{1, l-1}} \times \Omega_{\mathcal{V}_{n}^{l, l}} \times\left\{\left.\boldsymbol{\sigma}\right|_{\mathcal{V}_{n}^{l+1, \mathrm{~B}}}\right\}\right)
$$

e

$$
\Omega_{l}^{+}\left(\boldsymbol{\sigma}^{\prime}\right)=\left(\left\{\left.\boldsymbol{\sigma}^{\prime}\right|_{\mathcal{V}_{1}^{1, l-1}}\right\} \times \Omega_{\mathcal{V}_{1}^{l, l}} \times \Omega_{\mathcal{V}_{1}^{l+1, \mathrm{~B}}}\right) \times \cdots \times\left(\left\{\left.\boldsymbol{\sigma}^{\prime}\right|_{\mathcal{V}_{n}^{1, l-1}}\right\} \times \Omega_{\mathcal{V}_{n}^{l, l}} \times \Omega_{\mathcal{V}_{n}^{l+1, \mathrm{~B}}}\right) .
$$

Além disso, definindo a aplicação $\varphi_{l}=\left(\varphi_{l, 1}, \ldots, \varphi_{l, n}\right)$ por

$$
\boldsymbol{\sigma}, \boldsymbol{\xi}, \boldsymbol{\eta} \mapsto \boldsymbol{\varphi}_{l}(\boldsymbol{\sigma}, \boldsymbol{\xi}, \boldsymbol{\eta}) \stackrel{\text { def }}{=}\left(\varphi_{l, 1}\left(\sigma_{1}, \xi_{1}, \eta_{1}\right), \ldots, \varphi_{l, n}\left(\sigma_{n}, \xi_{n}, \eta_{n}\right)\right),
$$

em que para cada $j \in \mathcal{I}_{n}$,

$$
\varphi_{l, j}\left(\sigma_{j}, \xi_{j}, \eta_{j}\right) \stackrel{\text { def }}{=} \sigma_{j, 1}, \ldots, \sigma_{j, l-1}, \xi_{j, l}, \eta_{j, l+1}, \ldots, \eta_{j, \mathrm{~B}}
$$

podemos escrever as somas no lado direito de (4.1.14) da seguinte forma:

$$
\begin{aligned}
\sum_{\boldsymbol{\eta} \in \Omega_{l}^{-}(\boldsymbol{\sigma})} e^{-\beta H(\boldsymbol{\eta})} & \sum_{\boldsymbol{\eta}^{\prime} \in \Omega_{l}^{+}\left(\boldsymbol{\sigma}^{\prime}\right)} e^{-\beta H\left(\boldsymbol{\eta}^{\prime}\right)} \\
= & \sum_{\boldsymbol{\eta} \in \Omega_{\mathcal{V}^{1}, l-1}} \sum_{\boldsymbol{\xi} \in \Omega_{\mathcal{V}^{l}, l}} e^{-\beta H\left(\boldsymbol{\varphi}_{l}(\boldsymbol{\eta}, \boldsymbol{\xi}, \boldsymbol{\sigma})\right)} \sum_{\boldsymbol{\xi}^{\prime} \in \Omega_{\mathcal{V}^{l, l}}} \sum_{\boldsymbol{\eta}^{\prime} \in \Omega_{\mathcal{V}^{l+1}, \mathrm{~B}}} e^{-\beta H\left(\boldsymbol{\varphi}_{l}\left(\boldsymbol{\sigma}^{\prime}, \boldsymbol{\xi}^{\prime}, \boldsymbol{\eta}^{\prime}\right)\right)} .
\end{aligned}
$$

Como $\left|\mathcal{V}^{k, k}\right| \leq \mathcal{S}=o(N)$ para todo $k \in \mathcal{I}_{\mathrm{B}}$, podemos estimar o lado direito de (4.1.30) tomando-se o máximo sobre todas as configurações $\boldsymbol{\xi}, \boldsymbol{\xi}^{\prime} \in \mathcal{V}^{l, l}$. Prosseguindo dessa forma, obtemos que

$$
(4.1 .30) \leq 2^{2 \mathcal{S}} \max _{\boldsymbol{\xi} \in \Omega_{\mathcal{V} l, l}} \sum_{\boldsymbol{\eta} \in \Omega_{\mathcal{V}^{1}, l-1}} e^{-\beta H\left(\boldsymbol{\varphi}_{l}(\boldsymbol{\eta}, \boldsymbol{\xi}, \boldsymbol{\sigma})\right)} \max _{\boldsymbol{\xi}^{\prime} \in \Omega_{\mathcal{V} l, l}} \sum_{\boldsymbol{\eta}^{\prime} \in \Omega_{\mathcal{V}^{l+1, B}}} e^{-\beta H\left(\boldsymbol{\varphi}_{l}\left(\boldsymbol{\sigma}^{\prime}, \boldsymbol{\xi}^{\prime}, \boldsymbol{\eta}^{\prime}\right)\right)} .
$$


Reunindo então (4.1.30) e (4.1.31), e definindo as variáveis aleatórias

$$
Z_{1, l}^{(1)}\left(\boldsymbol{\xi}, \boldsymbol{\zeta}^{\prime}\right) \stackrel{\text { def }}{=} \sum_{\boldsymbol{\eta} \in \Omega_{\mathcal{V}^{1, l-1}}} e^{-\beta H\left(\boldsymbol{\varphi}_{l}\left(\boldsymbol{\eta}, \boldsymbol{\xi}, \boldsymbol{\zeta}^{\prime}\right)\right)}
$$

$\mathrm{e}$

$$
Z_{l, \mathrm{~B}}^{(1)}\left(\boldsymbol{\zeta}, \boldsymbol{\xi}^{\prime}\right) \stackrel{\text { def }}{=} \sum_{\boldsymbol{\eta}^{\prime} \in \Omega_{\mathcal{V}^{l}+1, \mathrm{~B}}} e^{-\beta H\left(\boldsymbol{\varphi}_{l}\left(\boldsymbol{\zeta}, \boldsymbol{\xi}^{\prime}, \boldsymbol{\eta}^{\prime}\right)\right)}
$$

concluímos que

$$
\begin{aligned}
& \max _{l \in\{2, \ldots, \mathrm{B}-1\}} \max _{\substack{e=\left(\boldsymbol{\sigma}, \boldsymbol{\sigma}^{\prime}\right) \in \mathcal{G} \\
\hat{\imath}(e)=l}} \sum_{\boldsymbol{\eta} \in \Omega_{l}^{-}(\boldsymbol{\sigma})} e^{-\beta H(\boldsymbol{\eta})} \sum_{\boldsymbol{\eta}^{\prime} \in \Omega_{l}^{+}\left(\boldsymbol{\sigma}^{\prime}\right)} e^{-\beta H\left(\boldsymbol{\eta}^{\prime}\right)}
\end{aligned}
$$

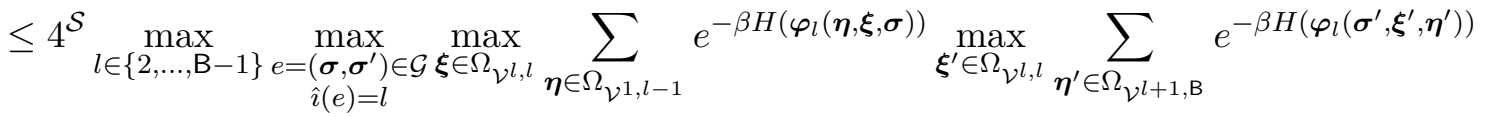

$$
\begin{aligned}
& =4^{\mathcal{S}} \max _{l \in\{2, \ldots, \mathrm{B}-1\}} \max _{\boldsymbol{\xi} \in \Omega_{\mathcal{V}^{l, l}}} \max _{\boldsymbol{\zeta}^{\prime} \in \Omega_{\mathcal{V}}} Z_{1, l}^{(1)}\left(\boldsymbol{\xi}, \boldsymbol{\zeta}^{\prime}\right) \max _{\boldsymbol{\xi}^{\prime} \in \Omega_{\mathcal{\nu}}, l} \max _{\boldsymbol{\zeta} \in \Omega_{\mathcal{V}}} Z_{l, \mathrm{~B}}^{(1)}\left(\boldsymbol{\zeta}, \boldsymbol{\xi}^{\prime}\right) .
\end{aligned}
$$

Observação 4.1.1. A consequência fundamental de toda a construção até aqui é que as variáveis $Z_{1, l}^{(1)}\left(\boldsymbol{\xi}, \boldsymbol{\zeta}^{\prime}\right)$ e $Z_{l, \mathrm{~B}}^{(1)}\left(\boldsymbol{\zeta}, \boldsymbol{\xi}^{\prime}\right)$ são funções de partição de GREMs com os mesmos parâmetros do GREM inicial, mas com um volume menor. Isto se tornará mais aparente na próxima seção.

\subsection{Estimativa Intermediária}

\subsubsection{Introdução}

Nesta seção, continuamos com a estimativa da variável aleatória $\Lambda_{1}$. Nosso foco aqui, no entanto, é estimar o termo

$$
\max _{l \in\{2, \ldots, \mathrm{B}-1\}} \max _{\boldsymbol{\xi} \in \Omega_{\mathcal{V}^{l, l}}} \max _{\boldsymbol{\zeta}^{\prime} \in \Omega_{\mathcal{V}}} Z_{1, l}^{(1)}\left(\boldsymbol{\xi}, \boldsymbol{\zeta}^{\prime}\right) \max _{\boldsymbol{\xi}^{\prime} \in \Omega_{\mathcal{V}}, l} \max _{\boldsymbol{\zeta} \in \Omega_{\mathcal{V}}} Z_{l, \mathrm{~B}}^{(1)}\left(\boldsymbol{\zeta}, \boldsymbol{\xi}^{\prime}\right)
$$

Como já foi antecipado anteriormente, as variáveis $Z_{1, l}^{(1)}\left(\boldsymbol{\xi}, \boldsymbol{\zeta}^{\prime}\right)$ e $Z_{l, \mathrm{~B}}^{(1)}\left(\boldsymbol{\zeta}, \boldsymbol{\xi}^{\prime}\right)$ podem ser vistas como funções de partição de GREMs, logo, a ideia inicial seria adaptar o que foi feito em [CCP87] na Proposição 3.2 e usar a desigualdade de Markov simples para estimá-las. Entretanto, essa desigualdade não nos dá uma probabilidade forte o suficiente para controlar o número exponencial de eventos que aparecem quando tomamos os sinais de max em (4.2.1).

A estratégia então é a seguinte. Inspirados no que foi feito na Seção III em [OP84], dividiremos o suporte da família de gaussianas $\mathscr{X}$, a saber, o espaço euclidiano $n$-dimensional em subconjuntos disjuntos $\Delta_{j_{1}, \ldots, j_{n}}$, e analisaremos separadamente as contribuições para a função de subpartição vindas de cada $\Delta_{j_{1}, \ldots, j_{n}}$ usando um caso particular da desigualdade de Chernoff, 
ao invés da desigualdade de Markov simples ou exponencial de Markov; esta última foi usada em [FIKP98]. Com esta proposta, é suficiente estudar a variável $Z_{1, l}^{(1)}\left(\boldsymbol{\xi}, \boldsymbol{\zeta}^{\prime}\right)$. Um argumento análogo valerá para $Z_{l, \mathrm{~B}}^{(1)}\left(\boldsymbol{\zeta}, \boldsymbol{\xi}^{\prime}\right)$.

\subsubsection{Estimativa para $Z_{1, l}^{(1)}\left(\boldsymbol{\xi}, \zeta^{\prime}\right)$}

Sejam $l \in\{2, \ldots, \mathrm{B}-1\}$ e $\boldsymbol{\xi} \in \Omega_{\mathcal{V} l, l}$ e $\boldsymbol{\zeta}^{\prime} \in \Omega_{\mathcal{V}}$ fixados. Com a notação (2.1.24), escrevemos

$$
Z_{1, l}^{(1)}\left(\boldsymbol{\xi}, \boldsymbol{\zeta}^{\prime}\right)=\sum_{\boldsymbol{\eta} \in \Omega_{\mathcal{V} 1, l-1}} e^{-\left\langle\mathfrak{m}^{*}, X_{\boldsymbol{\varphi}_{l}\left(\boldsymbol{\eta}, \boldsymbol{\xi}, \boldsymbol{\zeta}^{\prime}\right)}\right\rangle \sqrt{V}},
$$

em que $\mathfrak{m}^{*}$ está dado em (2.1.45).

Seja $M \in \mathbb{N}$ e vamos escrever

$$
\delta_{0}=\frac{1}{M}
$$

Agora, seja

$$
\mathbb{R}^{n}=\bigcup_{j_{1}=0}^{M+1} \cdots \bigcup_{j_{n}=0}^{M+1} \Delta_{j_{1}, \ldots, j_{n}}, \quad \operatorname{com} \Delta_{j_{1}, \ldots, j_{n}} \stackrel{\text { def }}{=} \Delta_{j_{1}}^{1} \times \cdots \times \Delta_{j_{n}}^{n},
$$

em que

$$
\Delta_{j_{k}}^{k} \stackrel{\text { def }}{=} \begin{cases}\left(-\infty, \delta_{0} \sqrt{P_{k}},\right. & \text { se } j_{k}=0 ; \\ \left(j_{k} \delta_{0} \sqrt{P_{k}},\left(j_{k}+1\right) \delta_{0} \sqrt{P_{k}}\right], & \text { se } j_{k}=1, \ldots, M ; \quad k \in \mathcal{I}_{n} . \\ \left(\left(1+\delta_{0}\right) \sqrt{P_{k}}, \infty\right), & \text { se } j_{k}=M+1,\end{cases}
$$

Notação. A fim de tornar a notação mais leve, para $j_{1}, \ldots, j_{n}$ fixado, denotaremos por $\bar{x}, \underline{x} \mathrm{e}$ $\tilde{x}$ os pontos de $\mathbb{R}^{n}$ dados por

$$
\bar{x}_{k} \stackrel{\text { def }}{=}\left(j_{k}+1\right) \delta_{0} \sqrt{P_{k}}, \quad \underline{x}_{k} \stackrel{\text { def }}{=} j_{k} \delta_{0} \sqrt{P_{k}} \quad \text { e } \quad \tilde{x}_{k} \stackrel{\text { def }}{=} \delta_{0} \sqrt{P_{k}} .
$$

Denotaremos também por $\mathcal{X}_{1}$ e $\mathcal{X}^{*}$ os seguintes conjuntos:

$$
\begin{aligned}
& \mathcal{X}_{1} \stackrel{\text { def }}{=}\left\{j_{1}, \ldots, j_{n}: 0 \leq j_{k} \leq M, \forall k \in \mathcal{I}_{n}\right\}, \\
& \mathcal{X}^{*} \stackrel{\text { def }}{=}\left\{j_{1}, \ldots, j_{n}: \exists k \in \mathcal{I}_{n} ; j_{k}=M+1\right\} .
\end{aligned}
$$

Por fim, denotaremos por $\alpha S$, o conjunto

$$
\alpha S \stackrel{\text { def }}{=}\{\alpha x: x \in S\}
$$


Com a notação anterior, escrevemos

$$
\begin{aligned}
Z_{1, l}^{(1)}\left(\boldsymbol{\xi}, \boldsymbol{\zeta}^{\prime}\right) & =\sum_{\boldsymbol{\eta} \in \Omega_{\mathcal{V}^{1}, l-1}}\left(\sum_{j_{1}, \ldots, j_{n}} \mathbb{1}\left\{-X_{\boldsymbol{\varphi}_{l}\left(\boldsymbol{\eta}, \boldsymbol{\xi}, \boldsymbol{\zeta}^{\prime}\right)} \in \sqrt{N} \Delta_{j_{1}, \ldots, j_{n}}\right\}\right) e^{-\left\langle\mathfrak{m}^{*}, X_{\boldsymbol{\varphi}_{l}\left(\boldsymbol{\eta}, \boldsymbol{\xi}, \boldsymbol{\zeta}^{\prime}\right)}\right\rangle \sqrt{V}} \\
& =\sum_{j_{1}, \ldots, j_{n}}\left(\sum_{\boldsymbol{\eta} \in \Omega_{\mathcal{V}^{1}, l-1}} \mathbb{1}\left\{-X_{\boldsymbol{\varphi}_{l}\left(\boldsymbol{\eta}, \boldsymbol{\xi}, \boldsymbol{\zeta}^{\prime}\right)} \in \sqrt{N} \Delta_{j_{1}, \ldots, j_{n}}\right\} e^{-\left\langle\mathfrak{m}^{*}, X_{\boldsymbol{\varphi}_{l}\left(\boldsymbol{\eta}, \boldsymbol{\xi}, \boldsymbol{\zeta}^{\prime}\right)}\right\rangle \sqrt{V}}\right) \\
& \leq S_{0}+S_{1}+S^{*},
\end{aligned}
$$

em que

$$
\begin{gathered}
S_{0} \stackrel{\text { def }}{=} \sum_{\boldsymbol{\eta} \in \Omega_{\mathcal{V}^{1}, l-1}} \mathbb{1}\left\{-X_{\boldsymbol{\varphi}_{l}\left(\boldsymbol{\eta}, \boldsymbol{\xi}, \boldsymbol{\zeta}^{\prime}\right)} \in \sqrt{N} \Delta_{0, \ldots, 0}\right\} e^{\left\langle\mathfrak{m}^{*}, \tilde{x}\right\rangle N}, \\
S^{*} \stackrel{\text { def }}{=} e^{-\beta H(\overline{\boldsymbol{\sigma}})} \sum_{j_{1}, \ldots, j_{n} \in \mathcal{X}^{*}}\left(\sum_{\boldsymbol{\sigma} \in \Omega_{\mathcal{V}}} \mathbb{1}\left\{-X_{\boldsymbol{\sigma}} \in \sqrt{N} \Delta_{j_{1}, \ldots, j_{n}}\right\}\right)
\end{gathered}
$$

e

$$
S_{1} \stackrel{\text { def }}{=} \sum_{m=1}^{n} \sum_{\left[k_{1}, \ldots, k_{m}\right]} \sum_{\substack{j_{1}, \ldots, j_{j} \in \mathcal{X}_{1} \\ j_{k_{1}}, \ldots, j_{k_{m}} \neq 0}}\left(\sum_{\boldsymbol{\eta} \in \Omega_{\mathcal{V}^{1}, l-1}} \mathbb{1}\left\{-X_{\boldsymbol{\varphi}_{l}\left(\boldsymbol{\eta}, \boldsymbol{\xi}, \boldsymbol{\zeta}^{\prime}\right)} \in \sqrt{N} \Delta_{j_{1}, \ldots, j_{n}}\right\}\right) e^{\left\langle\mathfrak{m}^{*}, \bar{x}\right\rangle N}
$$

em que a soma $\sum_{\left[k_{1}, \ldots, k_{m}\right]}$ está sobre todas as sequências de naturais satisfazendo que $1 \leq k_{1}<$ $\cdots<k_{m} \leq n$. Note que na desigualdade também usamos que $V \leq N$.

Estimativa para $S_{1}$. Começaremos observando que, como $\bar{x}=\underline{x}+\tilde{x}$, então por (2.1.23), $(4.2 .6)$ e $(2.1 .47)$

$$
\left\langle\mathfrak{m}^{*}, \bar{x}\right\rangle=\left\langle\mathfrak{m}^{*}, \underline{x}\right\rangle+\left\langle\mathfrak{m}^{*}, \tilde{x}\right\rangle \leq\left\langle\mathfrak{m}^{*}, \underline{x}\right\rangle+n \beta \beta_{\star} \delta_{0} .
$$

Daí,

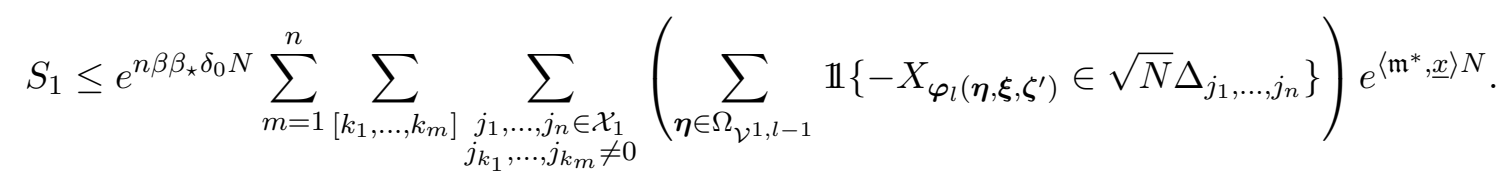

Observação 4.2.1. Note que, em (4.2.14), o ponto $\underline{x}=\left(\underline{x}_{1}, \ldots, \underline{x}_{n}\right)$ é tal que $\underline{x}_{i}=0$ para todo $i \neq k_{1}, \ldots, k_{m}$.

Seja

$$
\mathcal{Z}_{\left[k_{1}, \ldots, k_{m}\right]}^{(1)}\left(l, \boldsymbol{\xi}, \boldsymbol{\zeta}^{\prime}\right) \stackrel{\text { def }}{=} \sum_{\substack{j_{1}, \ldots, j_{n} \in \mathcal{X}_{1} \\ j_{k_{1}}, \ldots, j_{k_{m}} \neq 0}} N_{j_{1}, \ldots, j_{n}} e^{\left\langle\mathfrak{m}^{*}, \underline{x}\right\rangle N},
$$


em que

$$
N_{j_{1}, \ldots, j_{n}}=N_{j_{1}, \ldots, j_{n}}\left(1, l, \boldsymbol{\xi}, \boldsymbol{\zeta}^{\prime}\right) \stackrel{\text { def }}{=} \sum_{\boldsymbol{\eta} \in \Omega_{\mathcal{V}^{1}, l-1}} \mathbb{1}\left\{-X_{\varphi_{l}\left(\boldsymbol{\eta}, \boldsymbol{\xi}, \boldsymbol{\zeta}^{\prime}\right)} \in \sqrt{N} \Delta_{j_{1}, \ldots, j_{n}}\right\}
$$

Com a notação acima, escrevemos (4.2.14) como

$$
S_{1} \leq e^{n \beta \beta_{\star} \delta_{0} N} \sum_{m=1}^{n} \sum_{\left[k_{1}, \ldots, k_{m}\right]} \mathcal{Z}_{\left[k_{1}, \ldots, k_{m}\right]}^{(1)}\left(l, \boldsymbol{\xi}, \boldsymbol{\zeta}^{\prime}\right) .
$$

Na subseção seguinte obteremos uma cota para a variável simples $N_{j_{1}, \ldots, j_{n}}$. Por consequência, também obteremos uma cota para $\mathcal{Z}_{\left[k_{1}, \ldots, k_{m}\right]}^{(1)}\left(l, \boldsymbol{\xi}, \boldsymbol{\zeta}^{\prime}\right)$ e $S_{1}$.

\subsubsection{Estimativa de Grandes Desvios}

Nesta subseção obteremos uma cota superior para a variável aleatória $N_{j_{1}, \ldots, j_{n}}$. A ferramenta chave que usaremos para derivar tal cota é um caso particular da desigualdade de Chernoff (cf. Apêndice B, Lema B.0.1).

Sejam $m \in \mathcal{I}_{n},\left[k_{1}, \ldots, k_{m}\right]$ e $j_{1}, \ldots, j_{n} \in \mathcal{X}_{1} \operatorname{com} j_{k_{1}}, \ldots, j_{k_{m}} \neq 0$ fixados. Vamos supor, sem perda de generalidade, que $l-1=\alpha \mathrm{B}$, para algum $0<\alpha<1$ (esta suposição será assumida pelo resto deste capítulo). Então,

$$
\mid \mathcal{V}^{1, l-1} \stackrel{(4.1 .15)}{=}(l-1) \sum_{j=1}^{n} \mathrm{v}_{j}=\alpha \mathrm{B} \sum_{j=1}^{n} \mathrm{v}_{j} \stackrel{(2.1 .12)}{=} \alpha V .
$$

Agora, vamos considerar uma subfamília de $2^{\alpha V_{1}}+2^{\alpha\left(V_{1}+V_{2}\right)}+\cdots+2^{\alpha V}$ variáveis aleatórias gaussianas padrão independentes em $\mathscr{X}$, digamos

$$
X_{i_{1}, \ldots, i_{r}}^{r} \sim \mathcal{N}(0,1), \quad \forall i_{r}=1, \ldots, 2^{\alpha V_{r}} ; r=1, \ldots, n
$$

Aqui, as variáveis aleatórias $X_{i_{1}, \ldots, i_{r}}^{r}$ farão, sem perda de generalidade, o papel de $X_{\varphi_{l}\left(\boldsymbol{\eta}, \boldsymbol{\xi}, \boldsymbol{\zeta}^{\prime}\right)}$. Então, escrevemos

$$
\begin{aligned}
N_{j_{1}, \ldots, j_{n}} & =\sum_{i_{1}=1}^{2^{\alpha V_{1}}} \cdots \sum_{i_{n}=1}^{2^{\alpha V_{n}}} \prod_{r=1}^{n} \mathbb{1}\left\{-X_{i_{1}, \ldots, i_{r}}^{r} \in \sqrt{N} \Delta_{j_{r}}^{r}\right\} \\
& \leq 2^{\alpha \sum_{j=k_{m}+1}^{n}} V_{j} \sum_{i_{1}=1}^{2^{\alpha V_{1}}} \cdots \sum_{i_{k_{m}}=1}^{2^{\alpha V_{k_{m}}}} \prod_{r=1}^{m} \mathbb{1}\left\{-X_{i_{1}, \ldots, i_{k_{r}}}^{k_{r}} \in \sqrt{N} \Delta_{j_{k_{r}}}^{k_{r}}\right\},
\end{aligned}
$$

em que usamos que $\mathbb{1}_{\Delta_{0}^{r}} \leq 1, \forall r \neq k_{1}, \ldots, k_{m}$.

Vamos definir

$$
V_{r}^{\star} \stackrel{\text { def }}{=} \sum_{j=k_{r-1}+1}^{k_{r}} V_{j} \quad \text { e } \quad p_{r}^{\star}=\sum_{j=k_{r-1}+1}^{k_{r}} p_{j},
$$


para $r=1, \ldots, m$, em que por convenção $k_{0} \equiv 0$.

Considere agora uma outra subfamília de $2^{\alpha V_{1}^{\star}}+2^{\alpha\left(V_{1}^{\star}+V_{2}^{\star}\right)}+\cdots+2^{\alpha\left(V_{1}^{\star}+\cdots+V_{m}^{\star}\right)}$ variáveis aleatórias gaussianas padrão independentes pertencente a subfamília em (4.2.19), digamos

$$
X_{i_{1}, \ldots, i_{r}}^{r} \sim \mathcal{N}(0,1), \quad \forall i_{r}=1, \ldots, 2^{\alpha V_{r}^{\star}} ; r=1, \ldots, m
$$

Então, denotando por

$$
N_{j_{k_{1}}, \ldots, j_{k_{m}}}^{\star} \stackrel{\text { def }}{=} N_{j_{k_{1}}, \ldots, j_{k_{m}}}^{\star}\left(1, l, \boldsymbol{\xi}, \boldsymbol{\zeta}^{\prime}\right)=\sum_{i_{1}=1}^{2^{\alpha V_{1}^{\star}}} \cdots \sum_{i_{m}=1}^{2^{\alpha V_{m}^{\star}}} \prod_{r=1}^{m} \mathbb{1}\left\{-X_{i_{1}, \ldots, i_{r}}^{r} \in \sqrt{N} \Delta_{j_{k_{r}}}^{k_{r}}\right\},
$$

e usando que $V_{j} \leq p_{j} N$, obtemos que

$$
N_{j_{1}, \ldots, j_{n}} \leq 2^{\alpha N \sum_{j=k_{m}+1}^{n} p_{j}} N_{j_{k_{1}}, \ldots, j_{k_{m}}}^{\star(2.1 .32)} \stackrel{\left(\frac{1}{2} \alpha N \bar{P}_{k_{m}}\right.}{=} N_{j_{k_{1}}, \ldots, j_{k_{m}}}^{\star}
$$

em que

$$
\bar{P}_{j} \stackrel{\text { def }}{=} \beta_{\star}^{2}-P_{j} .
$$

Portanto,

$$
\mathcal{Z}_{\left[k_{1}, \ldots, k_{m}\right]}^{(1)}\left(l, \boldsymbol{\xi}, \boldsymbol{\zeta}^{\prime}\right) \leq e^{\frac{1}{2} \alpha N \bar{P}_{k_{m}}} \sum_{\substack{j_{1}, \ldots, j_{n} \in \mathcal{X}_{1} \\ j_{k_{1}}, \ldots, j_{k_{m}} \neq 0}} N_{j_{k_{1}}, \ldots, j_{k_{m}}}^{\star} e^{\left\langle\mathfrak{m}^{*}, \underline{x}\right\rangle N}
$$

Naturalmente, o passo agora é estimar a variável $N_{j_{k_{1}}, \ldots, j_{k_{m}}}^{\star}$. Começaremos com algumas notações adicionais.

Dada uma variável aleatória $X \sim \mathcal{N}(0,1)$, vamos denotar por

$$
q_{j_{k_{r}}}^{\star} \stackrel{\text { def }}{=} \mathbb{P}\left[-X \in \sqrt{N} \Delta_{j_{k_{r}}}^{k_{r}}\right], \quad r \in \mathcal{I}_{m}
$$

Para todo $N$ grande o suficiente, seguem duas observações: por um lado,

$$
q_{j_{k_{r}}}^{\star}=\int_{\underline{x}_{k_{r}} \sqrt{N}}^{\bar{x}_{k_{r}} \sqrt{N}} \frac{1}{\sqrt{2 \pi}} e^{-\frac{1}{2} x^{2}} d x \leq \int_{\underline{x}_{k_{r}} \sqrt{N}}^{\infty} e^{-\frac{1}{2} x^{2}} d x \leq e^{-\frac{1}{2} \underline{x}_{k_{r}}^{2} N}
$$

e por outro lado,

$$
q_{j_{k_{r}}}^{\star}=\int_{\underline{x}_{k_{r}} \sqrt{N}}^{\bar{x}_{k_{r}} \sqrt{N}} \frac{1}{\sqrt{2 \pi}} e^{-\frac{1}{2} x^{2}} d x \geq \frac{\tilde{x}_{k_{r}} \sqrt{N}}{\sqrt{2 \pi}} e^{-\frac{1}{2} \bar{x}_{k_{r}}^{2} N} \geq e^{-\frac{1}{2} \bar{x}_{k_{r}}^{2} N} .
$$

Como é comum em problemas como esse, gostaríamos de estimar a variável $N_{j_{k_{1}}, \ldots, j_{k_{m}}}^{\star}$ por alguma quantidade da ordem de sua média

$$
\mathbb{E}\left[N_{j_{k_{1}}, \ldots, j_{k_{m}}}^{\star}\right]=\prod_{r=1}^{m} q_{j_{k_{r}}}^{\star} 2^{\alpha V_{r}^{\star}}
$$


Entretanto, fixados $1 \leq r \leq s \leq m$, não é difícil ver que para certos índices $j_{k_{1}}, \ldots, j_{k_{m}}$,

$$
\prod_{i=r}^{s} q_{j_{k_{i}}}^{\star} 2^{\alpha V_{i}^{\star}} \stackrel{(4.2 .28)}{\leq} e^{\frac{1}{2} \sum_{i=r}^{s}\left(\alpha \beta_{\star}^{2} V_{i}^{\star}-\underline{x}_{k_{i}}^{2}\right)} \downarrow 0
$$

quando $N$ cresce, e isto não deveria ser verdade já que as variáveis $X_{i_{1}}^{1}, \ldots, X_{i_{1}, \ldots, i_{m}}^{m}$ estão suportadas em $\mathcal{A}_{n}(N, \delta)$. Então, procedendo como gostaríamos, estaríamos negligenciando contribuições para $Z_{1, l}^{(1)}\left(\boldsymbol{\xi}, \boldsymbol{\zeta}^{\prime}\right)$. A fim de corrigir esse problema, estimamos $N_{j_{k_{1}}, \ldots, j_{k_{m}}}^{\star}$ como segue.

Definição 4.2.1. Seja $c_{u}>0$ uma constante a ser escolhida depois. Vamos definir a seguinte família de inteiros. Seja $J_{0} \stackrel{\text { def }}{=} 0$ e recursivamente, defina

$$
J_{1} \stackrel{\text { def }}{=} \begin{cases}\inf \left\{0<r \leq m: \prod_{i=1}^{r} q_{j_{k}}^{\star} 2^{\alpha V_{i}^{\star}}<c_{u} N\right\}, & \text { se tal inteiro existe; } \\ m+1, & \text { c.c. }\end{cases}
$$

Se $J_{1}=m$, defina $J_{2}=m+1$. Caso contrário, defina

$$
J_{2} \stackrel{\text { def }}{=} \begin{cases}\inf \left\{J_{1}<r \leq m: \prod_{i=J_{1}+1}^{r} q_{j_{k}}^{\star} 2^{\alpha V_{i}^{\star}}<c_{u} N\right\}, & \text { se tal inteiro existe; } \\ m+1, & \text { c.c. }\end{cases}
$$

Mais geralmente, se $J_{q-1}=m$, defina $J_{q}=m+1$. Caso contrário, defina

$$
J_{q} \stackrel{\text { def }}{=} \begin{cases}\inf \left\{J_{q-1}<r \leq m: \prod_{i=J_{q-1}+1}^{r} q_{j_{k_{i}}}^{\star} 2^{\alpha V_{i}^{\star}}<c_{u} N\right\}, & \text { se tal inteiro existe; } \\ m+1, & \text { c.c. }\end{cases}
$$

Vamos denotar por $q(m)$ o inteiro pertencente ao conjunto $\{1, \ldots, m+1\}$ tal que $J_{q(m)}=$ $m+1$.

Agora, para cada $r \in \mathcal{I}_{m}$, defina

$$
\rho_{j_{k_{1}}, \ldots, j_{k_{r}}}^{\star} \stackrel{\text { def }}{=} \begin{cases}4, & \text { se } r \neq J_{1}, \ldots, J_{q(m)-1} ; \\ 4 c_{u} N\left(\prod_{i=J_{s-1}+1}^{J_{s}} q_{j_{k_{i}}}^{\star} 2^{\alpha V_{i}^{\star}}\right)^{-1}, & \text { se } r=J_{s} \text { para algum } s=1, \ldots, q(m)-1 .\end{cases}
$$

Observação 4.2.2. Note que, dado $m \in \mathcal{I}_{n}$,

$$
0=J_{0}<J_{1}<J_{2}<\cdots<J_{q(m)-1}<J_{q(m)}=m+1
$$

e para todo $s=1, \ldots, q(m)$,

$$
\prod_{i=J_{s-1}+1}^{r} q_{j_{i}}^{\star} 2^{\alpha V_{i}^{\star}} \geq c_{u} N, \quad \forall r \in\left\{J_{s-1}+1, \ldots, J_{s}-1\right\} .
$$


Proposição 4.2.1. Com a notação anterior, para qualquer constante $c_{u}>0$ e todo $0 \neq$ $j_{k_{1}}, \ldots, j_{k_{m}} \leq M$

$$
\mathbb{P}\left[N_{j_{k_{1}}, \ldots, j_{k_{m}}}^{\star}>\prod_{r=1}^{m} \rho_{j_{k_{1}}, \ldots, j_{k_{r}}}^{\star} q_{j_{k_{r}}}^{\star} 2^{\alpha V_{r}^{\star}}\right] \leq m e^{-c_{u} N}
$$

Demonstração. Provaremos esta proposição argumentando por indução sobre $m \in \mathcal{I}_{n}$. Começaremos definindo o evento

$$
A_{m} \stackrel{\text { def }}{=}\left\{N_{j_{k_{1}}, \ldots, j_{k_{m}}}^{\star}>b_{m}\right\}
$$

em que

$$
b_{m} \stackrel{\text { def }}{=} \prod_{r=1}^{m} \rho_{j_{k_{1}}, \ldots, j_{k_{r}}}^{\star} q_{j_{k_{r}}}^{\star} 2^{\alpha V_{r}^{\star}} .
$$

Lema 4.2.1. Para todo $m \in \mathcal{I}_{n}$,

$$
\vartheta_{m} \stackrel{\text { def }}{=}\left(\rho_{j_{k_{1}}, \ldots, j_{k_{m}}}^{\star}-1\right) b_{m-1} q_{j_{k_{m}}}^{\star} 2^{\alpha V_{m}^{\star}} \geq 3 c_{u} N
$$

em que $b_{0} \equiv 1$.

Demonstração. De fato, primeiro observe que $\rho_{j_{k_{1}}, \ldots, j_{k_{r}}}^{\star} \geq 4$ para todo $r \in \mathcal{I}_{m}$. Isto segue como consequência da definição de $J_{1}, \ldots, J_{q(m)}$. Assim, escrevemos $\vartheta_{m}$ como

$$
\begin{aligned}
\vartheta_{m} & =\left(1-\frac{1}{\rho_{j_{k_{1}}, \ldots, j_{k_{m}}}^{\star}}\right) \prod_{r=1}^{m} \rho_{j_{k_{1}}, \ldots, j_{k_{r}}}^{\star} q_{j_{k_{r}}}^{\star} 2^{\alpha V_{r}^{\star}} \\
& \geq \frac{3}{4} \cdot \prod_{r=1}^{m} \rho_{j_{k_{1}}, \ldots, j_{k_{r}}}^{\star} q_{j_{k_{r}}}^{\star} 2^{\alpha V_{r}^{\star}} .
\end{aligned}
$$

Agora, de (4.2.35), se $J_{q(m)-1}<m$, temos que

$$
\begin{aligned}
\prod_{r=1}^{m} \rho_{j_{k_{1}}, \ldots, j_{k_{r}}}^{\star} q_{j_{k_{r}}}^{\star} 2^{\alpha V_{r}^{\star}} & =4^{m-q(m)+1}\left(4 c_{u} N\right)^{q(m)-1} \underbrace{\prod_{r=J_{q(m)-1}+1}^{m} q_{j_{k_{r}}}^{\star} 2^{\alpha V_{r}^{\star}}}_{\geq c_{u} N} \\
& \geq 4^{m}\left(c_{u} N\right)^{q(m)} \\
& \geq c_{u} N .
\end{aligned}
$$

Se $J_{q(m)-1}=m$ temos que

$$
\prod_{r=1}^{m} \rho_{j_{k_{1}}, \ldots, j_{k_{r}}}^{\star} q_{j_{k_{r}}}^{\star} 2^{\alpha V_{r}^{\star}}=4^{m-q(m)+1}\left(4 c_{u} N\right)^{q(m)-1}=\left(4 c_{u} N\right)^{m} \geq c_{u} N
$$

Juntando (4.2.42), (4.2.43) e (4.2.44), obtemos (4.2.41). 
Voltando à prova da proposição, vamos supor inicialmente que $m=1$. Neste caso, como $N_{j_{k_{1}}}^{\star}$ é a soma de $2^{\alpha V_{1}^{\star}}$ v.a.'s independentes e identicamente distribuídas segundo uma v.a. Bernoulli de parâmetro $q_{j_{k_{1}}}^{\star}$, invocando o Lema B.0.1 $\operatorname{com} \theta=\rho_{j_{k_{1}}}^{\star} \geq 4$, segue que

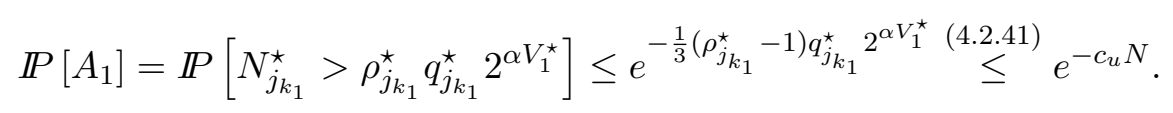

Agora, suponhamos que (4.2.38) valha para $m-1$, ou seja,

$$
\mathbb{P}\left[A_{m-1}\right] \leq(m-1) e^{-c_{u} N} .
$$

Vamos mostrar que (4.2.38) também vale para $m$. Com efeito, seja

$$
I_{m-1} \stackrel{\text { def }}{=}\left\{\left(i_{1}, \ldots, i_{m-1}\right):-X_{i_{1}, \ldots, i_{r}}^{r} \in \sqrt{N} \Delta_{j_{k_{r}}}^{k_{r}}, \forall r=1, \ldots, m-1\right\} .
$$

Então, usando a independência entre as variáveis aleatórias $X_{i_{1}}^{1}, \ldots, X_{i_{1}, \ldots, i_{m}}^{m}$, segue que

$$
\begin{aligned}
& \mathbb{P}\left[A_{m}\right]=\underbrace{\mathbb{P}\left[A_{m} \mid A_{m-1}\right]}_{\leq 1} \mathbb{P}\left[A_{m-1}\right]+\mathbb{P}\left[A_{m} \mid A_{m-1}^{c}\right] \underbrace{\mathbb{P}\left[A_{m-1}^{c}\right]}_{\leq 1} \\
& \leq \mathbb{P}\left[A_{m-1}\right]+\mathbb{P}\left[\sum_{\left(i_{1}, \ldots, i_{m-1}\right) \in I_{m-1}} \sum_{i_{m}=1}^{2^{\alpha V_{m}^{\star}}} \mathbb{1}\left\{-X_{i_{1}, \ldots, i_{m}}^{m} \in \sqrt{N} \Delta_{j_{k m}}^{k_{m}}\right\}>b_{m} \mid A_{m-1}^{c}\right] \\
& \stackrel{(\text { H.I.) }}{\leq}(m-1) e^{-c_{u} N}+\underbrace{\mathbb{P}\left[\sum_{i_{0}=1}^{b_{m-1} 2^{\alpha V_{m}^{\star}}} \mathbb{1}\left\{-X_{i_{0}} \in \sqrt{N} \Delta_{j_{m}}^{k_{m}}\right\}>b_{m}\right]}_{\stackrel{\text { def }}{=} \mathbb{P}\left[A_{m}^{\star}\right]},
\end{aligned}
$$

em que o conjunto $\left\{X_{i_{0}}\right\}$ é apenas um reordenamento das variáveis em $\left\{X_{i_{1}, \ldots, i_{m}}^{m}\right\}$. Observe agora que é suficiente mostrarmos que $\mathbb{P}\left[A_{m}^{\star}\right] \leq e^{-c_{u} N}$ para a conclusão da prova da proposição. Neste caso, observe primeiro que $b_{m}=\rho_{j_{k_{1}}, \ldots, j_{k_{m}}}^{\star} b_{m-1} q_{j_{k_{m}}}^{\star} 2^{\alpha V_{m}^{\star}}$. Agora, no evento $A_{m}^{\star}$ temos uma soma de $b_{m-1} 2^{\alpha V_{m}^{\star}}$ v.a.'s independentes e identicamente distribuídas segundo uma v.a. Bernoulli de parâmetro $q_{j_{k m}}^{\star}$. Invocando novamente o Lema B.0.1, mas, desta vez, com $\theta=\rho_{j_{k_{1}}, \ldots, j_{k_{m}}}^{\star} \geq 4$, obtemos que

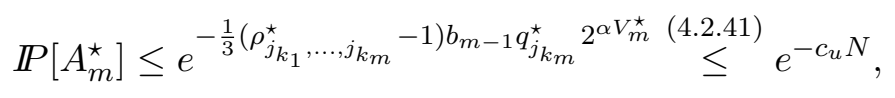

encerrando a demonstração.

Como consequência imediata da Proposição 4.2.1, temos o seguinte corolário.

Corolário 4.2.1. Dado $\varepsilon>0$, se $c_{u}>\log 2+2 \varepsilon$, então

$$
\mathbb{P}\left[\bigcup_{l \in \mathcal{I}_{\mathrm{B}}} \bigcup_{\boldsymbol{\xi} \in \Omega_{\mathcal{V}}, l} \bigcup_{\boldsymbol{\zeta}^{\prime} \in \Omega_{\mathcal{V}}} \bigcup_{m \in \mathcal{I}_{n}} \bigcup_{\left[k_{1}, \ldots, k_{m}\right]} \bigcup_{\substack{j_{1}, \ldots, j_{n} \in \mathcal{X}_{1} \\ j_{k_{1}}, \ldots, j_{k_{m}} \neq 0}} N_{j_{k_{1}}, \ldots, j_{k_{m}}}^{\star}\left(1, l, \boldsymbol{\xi}, \boldsymbol{\zeta}^{\prime}\right)>b_{m}\right] \leq e^{-\varepsilon N},
$$

para todo $N$ grande o suficiente, em que $b_{m}$ está definido em (4.2.40). 
Demonstração. De fato, relembremos inicialmente que a estimativa em (4.2.38) foi feita para $N_{j_{k_{1}}, \ldots, j_{k_{m}}}^{\star}=N_{j_{k_{1}}, \ldots, j_{k_{m}}}^{\star}\left(1, l, \boldsymbol{\xi}, \boldsymbol{\zeta}^{\prime}\right)$ com todas as variáveis, $l \in \mathcal{I}_{\mathrm{B}}, \boldsymbol{\xi} \in \Omega_{\mathcal{V}^{l, l},}, \boldsymbol{\zeta}^{\prime} \in \Omega_{\mathcal{V}}, m \in \mathcal{I}_{n}$, $\left[k_{1}, \ldots, k_{m}\right]$ e $0 \neq j_{k_{1}}, \ldots, j_{k_{m}} \leq M$ fixadas. Como não há mais que $\mathrm{B}(M+1)^{n} 2^{\mathcal{S}} 2^{V}$ delas, pela Proposição 4.2.1, o lado esquerdo de (4.2.49) é cotado por

$$
n \mathrm{~B}(M+1)^{n} 2^{\mathcal{S}} 2^{V} e^{-c_{u} N} \leq n \mathrm{~B}(M+1)^{n} 2^{\mathcal{S}} e^{-2 \varepsilon N} \leq e^{-\varepsilon N},
$$

para todo $N \geq N_{0}$, em que $N_{0}=N_{0}(\varepsilon) \in \mathbb{N}$ é escolhido tal que $n \mathrm{~B}(M+1)^{n} 2^{\mathcal{S}} e^{-\varepsilon N_{0}} \leq 1$. Isso encerra a demonstração.

\subsubsection{Estimativa Determinística}

Por causa de (4.2.26) e do Corolário 4.2.1, a cota que estamos procurando para $S_{1}$ mora no termo

$$
e^{\frac{1}{2} \alpha N \bar{P}_{k_{m}}} \sum_{\substack{j_{1}, \ldots, j_{n} \in \mathcal{X}_{1} \\ j_{k_{1}}, \ldots, j_{k_{m}} \neq 0}}\left(\prod_{r=1}^{m} \rho_{j_{k_{1}}, \ldots, j_{k_{r}}}^{\star} q_{j_{k_{r}}}^{\star} 2^{\alpha V_{r}^{\star}}\right) e^{\left\langle\mathfrak{m}^{*}, \underline{x}\right\rangle N}
$$

que evidentemente é não aleatório. Nosso objetivo nesta subseção é derivar uma cota para (4.2.51) uniforme com respeito às variáveis $m \in \mathcal{I}_{n} \mathrm{e}\left[k_{1}, \ldots, k_{m}\right]$.

De acordo com a Definição 4.2 .1 , para cada $m \in \mathcal{I}_{n}$ e cada $\left[k_{1}, \ldots, k_{m}\right]$ fixados, o conjunto de índices sobre o qual a soma em (4.2.51) está pode ser naturalmente decomposto em uma união de $m+1$ subconjuntos disjuntos, digamos $\left\{\mathbb{I}_{m, \alpha}^{\star}(s)\right\}_{s=0}^{m}$, definidos como

$$
\mathbb{I}_{m, \alpha}^{\star}(s)=\mathbb{I}_{m, \alpha}^{\star}(s)\left[k_{1}, \ldots, k_{m}\right] \stackrel{\text { def }}{=}\left\{j_{1}, \ldots, j_{n} \in \mathcal{X}_{1}: j_{k_{1}}, \ldots, j_{k_{m}} \neq 0 \text { e } J_{q(m)-1}=s\right\} .
$$

Por consequência, para todo $j_{1}, \ldots, j_{n} \in \mathbb{I}_{m, \alpha}^{\star}(s)$, é imediato que

$$
\begin{gathered}
\prod_{r=1}^{m} \rho_{j_{k_{1}}, \ldots, j_{k_{r}}} q_{j_{k_{r}}}^{\star} 2^{\alpha V_{r}^{\star}} \stackrel{(}{\stackrel{4.2 .35)}{=}} 4^{m}\left(c_{u} N\right)^{s}\left(1+\mathbb{1}_{\{0 \leq s<m\}} \prod_{r=s+1}^{m} q_{j_{k_{r}}} 2^{\alpha V_{r}^{\star}}\right) \\
\stackrel{(4.2 .28)}{\leq}\left(4 c_{u} N\right)^{m}\left(1+\mathbb{1}\{0 \leq s<m\} \prod_{r=s+1}^{m} e^{-\frac{1}{2} \underline{x}_{k_{r}}^{2} N_{2} \alpha V_{r}^{\star}}\right) \\
\stackrel{(2.1 .32)}{=}\left(4 c_{u} N\right)^{m} e^{\frac{N}{2}\left(\sum_{r=s+1}^{m}\left(\alpha \beta_{\star}^{2} p_{r}^{\star}-\underline{x}_{k_{r}}^{2}\right)\right) \mathbb{1}\{s \neq m\}},
\end{gathered}
$$

para todo $N$ grande o suficiente.

Portanto,

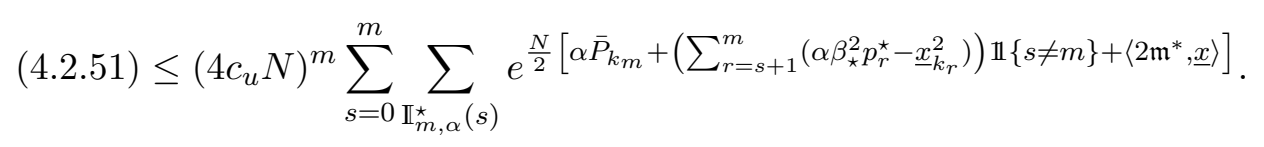


Notação. Vamos denotar por

$$
\begin{aligned}
S_{m, \alpha}^{\star}(s) & =S_{m, \alpha}^{\star}(s)\left[k_{1}, \ldots, k_{m}\right] \\
& \stackrel{\text { def }}{=} \sum_{\mathbb{I}_{m, \alpha}^{\star}(s)} e^{\frac{N}{2}\left[\alpha \bar{P}_{k_{m}}+\left(\sum_{r=s+1}^{m}\left(\alpha \beta_{\star}^{2} p_{r}^{\star}-\underline{x}_{k_{r}}^{2}\right)\right) \mathbb{1}\{s \neq m\}+\left\langle 2 \mathfrak{m}^{*}, \underline{x}\right\rangle\right],}
\end{aligned}
$$

para $s=0, \ldots, m$.

A fim de obter alguma intuição para os próximos passos, é importante que o leitor observe a semelhança entre os exponentes das exponenciais em (4.2.55) e (3.1) de [CCP87] (cf. Proposição 3.2). As estimativas seguintes estão inspiradas na prova da Proposição 3.3 em [CCP87] e feita com mais detalhes em [Pic92] (cf. páginas 192-193).

O próximo passo será estimar o termo $S_{m, \alpha}^{\star}(s)$. Primeiro, lidaremos com os casos $s=$ $0, \ldots, m-1$. O caso $s=m$ será estudado em separado.

Estimativa para $S_{m, \alpha}^{\star}(s)$ com $s \in\{0, \ldots, m-1\}$. Começaremos fixando $j_{1}, \ldots, j_{n} \in \mathbb{I}_{m, \alpha}^{\star}(s)$ e introduzindo algumas novas notações.

Notação. Dados $0 \leq i \leq j \leq n$, vamos definir a projeção $\Pi_{i}^{j}: \mathbb{R}^{n} \rightarrow \mathbb{R}^{j-i}$ como a função que apaga todas as coordenadas fora do conjunto $\{i+1, \ldots, j\}$, isto é,

$$
x \mapsto \Pi_{i}^{j} x \stackrel{\text { def }}{=}\left(x_{i+1}, \ldots, x_{j}\right),
$$

com a convenção $\Pi_{j}^{j} \equiv 0$.

Relembrando que $\underline{x}$ é tal que $\underline{x}_{i}=0, \forall i \neq k_{1}, \ldots, k_{m}$ (cf. Observação 4.2.1), escrevemos

$$
\begin{gathered}
\alpha \bar{P}_{k_{m}}+\sum_{r=s+1}^{m}\left(\alpha \beta_{\star}^{2} p_{r}^{\star}-\underline{x}_{k_{r}}^{2}\right) \stackrel{(4.2 .21)}{=} \alpha \bar{P}_{k_{m}}+\alpha \beta_{\star}^{2} \sum_{r=s+1}^{m} \sum_{j=k_{r-1}+1}^{k_{r}} p_{j}-\sum_{r=s+1}^{m} \underline{x}_{k_{r}}^{2} \\
\stackrel{(4.2 .25)}{=} \alpha \bar{P}_{k_{s}}-\sum_{r=s+1}^{m} \underline{x}_{k_{r}}^{2} \\
=\alpha \bar{P}_{k_{s}}-\left\langle\Pi_{k_{s}}^{n} \underline{x}, \Pi_{k_{s}}^{n} \underline{x}\right\rangle,
\end{gathered}
$$

e daí,

$$
S_{m, \alpha}^{\star}(s)=\sum_{\mathbb{I}_{m, \alpha}^{\star}(s)} e^{\frac{N}{2}\left[\alpha \bar{P}_{k_{s}}+\left\langle 2 \mathfrak{m}^{*}, \underline{x}\right\rangle-\left\langle\Pi_{k_{s}}^{n}, \Pi_{k_{s}}^{n} \underline{x}\right\rangle\right]} .
$$

Agora, note que

$$
\begin{aligned}
\left\langle 2 \mathfrak{m}^{*}, \underline{x}\right\rangle-\left\langle\Pi_{k_{s}}^{n} \underline{x}, \Pi_{k_{s}}^{n} \underline{x}\right\rangle= & \left\langle 2 \Pi_{0}^{k_{s}} \mathfrak{m}^{*}, \Pi_{0}^{k_{s}} \underline{x}\right\rangle \\
=\left\langle 2 \Pi_{0}^{k_{s}} \mathfrak{m}^{*}, \Pi_{0}^{k_{s}} \underline{x}\right\rangle & +\left\langle\Pi_{k_{s}}^{n} \mathfrak{m}^{*}-\Pi_{k_{s}}^{n} \mathfrak{m}^{*}, \Pi_{k_{s}}^{n} \mathfrak{m}^{*}\right\rangle \\
& \quad-\left\langle\Pi_{k_{s}}^{n} \mathfrak{m}^{*}-\Pi_{k_{s}}^{n} \underline{x}\right\rangle \\
= & \left.\left\langle 2 \Pi_{0}^{k_{s}} \mathfrak{m}^{*}, \Pi_{0}^{k_{s}} \underline{x}\right\rangle+\left\|\Pi_{k_{s}}^{n} \mathfrak{m}^{*}\right\|^{2}-\| \Pi_{k_{s}}^{n} \mathfrak{m}^{*}-\Pi_{k_{s}}^{n} \underline{x}\right\rangle \|^{2} .
\end{aligned}
$$


Portanto, para $m=0, \ldots, m-1$,

$$
S_{m, \alpha}^{\star}(s)=\sum_{\mathbb{I}_{m, \alpha}^{\star}(s)} e^{\frac{N}{2}\left[\alpha \bar{P}_{k_{s}}+\left\langle 2 \Pi_{0}^{k_{s}} \mathfrak{m}^{*}, \Pi_{0}^{k_{s}} \underline{x}\right\rangle+\left\|\Pi_{k_{s}}^{n} \mathfrak{m}^{*}\right\|^{2}-\left\|\Pi_{k_{s}}^{n} \mathfrak{m}^{*}-\Pi_{k_{s}}^{n} \underline{x}\right\|^{2}\right] .}
$$

Para a sequência seguinte vamos precisar das seguintes notações adicionais.

Notação: Para $j \in\{0, \ldots, n\}$, seja $\Psi_{j}$ o subconjunto de $\mathbb{R}^{j}$ obtido de $\Psi$ pela aplicação da projeção $\Pi_{0}^{j}$, isto é,

$$
\Psi_{j} \stackrel{\text { def }}{=} \Pi_{0}^{j} \Psi=\left\{\Pi_{0}^{j} y: y \in \Psi\right\}
$$

e defina o funcional $\phi_{j}: \Psi_{j} \rightarrow[0, \infty)$ como

$$
x \mapsto \phi_{j}(x) \stackrel{\text { def }}{=}\left\langle x, \Pi_{0}^{j} \mathfrak{m}^{*}\right\rangle .
$$

Por último, com um pouco de abuso de notação, seja o conjunto $\Psi_{j}^{n}(\alpha)$ dado por

$$
\Psi_{j}^{n}(\alpha) \stackrel{\text { def }}{=}\left\{x \in \mathbb{R}^{n-j}: \forall k=j+1, \ldots, n, \sum_{i=j+1}^{k} x_{i}^{2} \leq \alpha \beta_{\star}^{2} \sum_{i=j+1}^{k} p_{i}\right\},
$$

em que convencionamos $\Psi_{n}^{n}(\alpha) \equiv\{0\}$. Por simplicidade de notação, o caso especial $\Psi_{0}^{n}(\alpha)$ será indicado simplesmente por $\Psi(\alpha)$. Note que $\Psi_{n} \equiv \Psi$.

Com as definições acima, seguem duas observações: $(i)$ A primeira vem do fato que para todo $j \in\{0, \ldots, n\}$ o conjunto $\Psi_{j}$ é compacto. Desse fato, combinando as Proposições A.0.1 e A.0.2, obtemos que $\phi_{j}$ é limitada e atinge seu valor máximo absoluto em algum ponto $\operatorname{de} \operatorname{ext} \Psi_{j} \neq \varnothing$, o conjunto dos pontos extremos de $\Psi_{j}$, digamos $\mathfrak{u}_{j}$, ou seja

$$
\max _{x \in \Psi_{j}} \phi_{j}(x)=\max _{x \in \operatorname{ext} \Psi_{j}} \phi_{j}(x)=\phi_{j}\left(\mathfrak{u}_{j}\right) .
$$

Como por construção $\underline{x} \in \Psi$ para todo $j_{1}, \ldots, j_{n} \in \mathbb{I}_{m, \alpha}^{\star}(s)$, então a projeção $\Pi_{0}^{k_{s}} \underline{x} \in \Psi_{k_{s}}$, e daí, configurando $j=k_{s}$ em (4.2.62), de (4.2.64) é imediato que

$$
\phi_{k_{s}}\left(\Pi_{0}^{k_{s}} \underline{x}\right) \leq \phi_{k_{s}}\left(\mathfrak{u}_{k_{s}}\right)
$$

(ii) A segunda observação segue por causa do fato que para todo $j \in\{0, \ldots, n\}$ o conjunto $\Psi_{j}^{n}(\alpha)$ é convexo e fechado. Neste caso, fixado $\Pi_{j}^{n} \mathfrak{m}^{*}$, pelo Teorema A.0.1, existe um único ponto em $\Psi_{j}^{n}(\alpha)$, digamos $\mathfrak{m}_{\alpha, j}$, tal que

$$
\mathrm{d}\left(\Pi_{j}^{n} \mathfrak{m}^{*}, \Psi_{j}^{n}(\alpha)\right)=\left\|\Pi_{j}^{n} \mathfrak{m}^{*}-\mathfrak{m}_{\alpha, j}\right\|
$$

Como a hipótese $s=J_{q(m)-1}$ implica (cf. (4.2.37)) que

$$
\forall i=s+1, \ldots, m, \quad c_{u} N \leq \prod_{r=s+1}^{i} q_{j_{k_{r}}}^{\star} 2^{\alpha V_{r}^{\star}} \stackrel{(4.2 .28)}{\leq} e^{\frac{N}{2} \sum_{r=s+1}^{i}\left(\alpha \beta_{\star}^{2} p_{r}^{\star}-\underline{x}_{k_{r}}^{2}\right)},
$$


então, se $N$ é grande o suficiente,

$$
\forall i=s+1, \ldots, m, \quad \sum_{r=s+1}^{i}\left(\alpha \beta_{\star}^{2} p_{r}^{\star}-\underline{x}_{k_{r}}^{2}\right) \geq 0 .
$$

Daí, como $\underline{x}$ é tal que $\underline{x}_{i}=0, \forall i \neq k_{1}, \ldots, k_{m}$, usando também (4.2.21), obtemos que

$$
\forall i=k_{s}+1, \ldots, n, \quad \sum_{j=k_{s}+1}^{i}\left(\alpha \beta_{\star}^{2} p_{j}-\underline{x}_{j}^{2}\right) \geq 0
$$

ou seja, $\Pi_{k_{s}}^{n} \underline{x} \in \Psi_{k_{s}}^{n}(\alpha)$. Então, pondo $j=k_{s}$ em (4.2.63), de (4.2.66) obtemos que

$$
\left\|\Pi_{k_{s}}^{n} \mathfrak{m}^{*}-\mathfrak{m}_{\alpha, k_{s}}\right\|^{2} \leq\left\|\Pi_{k_{s}}^{n} \mathfrak{m}^{*}-\Pi_{k_{s}}^{n} \underline{x}\right\|^{2}
$$

para todo $N$ grande o suficiente.

Portanto, juntando (4.2.65) e (4.2.70), para todo $s=0, \ldots, m-1$, obtemos que

$$
S_{m, \alpha}^{\star}(s) \leq M^{m} e^{\frac{N}{2}\left[2 \phi_{k_{s}}\left(\mathfrak{u}_{k_{s}}\right)+\alpha \bar{P}_{k_{s}}+\left\|\Pi_{k_{s}}^{n} \mathfrak{m}^{*}\right\|^{2}-\left\|\Pi_{k_{s}}^{n} \mathfrak{m}^{*}-\mathfrak{w}_{\alpha, k_{s}}\right\|^{2}\right]}
$$

para todo $N$ grande o suficiente.

Notação. Dados $j \in\{0, \ldots, n\}, \beta>0$ e $0 \leq \alpha \leq 1$, vamos definir

$$
2 F_{n, j}(\beta, \alpha) \stackrel{\text { def }}{=} \alpha \bar{P}_{j}+\left\|\Pi_{j}^{n} \mathfrak{m}^{*}\right\|^{2}-\left\|\Pi_{j}^{n} \mathfrak{m}^{*}-\mathfrak{m}_{\alpha, j}\right\|^{2},
$$

com a convenção $F_{n, n} \equiv 0$. Quando $\alpha=1, F_{n, j}(\beta, 1)$ será indicado simplesmente por $F_{n, j}(\beta)$. Neste caso, note que $F_{n, 0}(\beta) \equiv F_{n}(\beta)$.

Observação 4.2.3. Alternativamente, usando as propriedades de produto interno, podemos escrever (4.2.72) como

$$
2 F_{n, j}(\beta, \alpha)=\alpha \bar{P}_{j}+2\left\langle\Pi_{j}^{n} \mathfrak{m}^{*}, \mathfrak{m}_{\alpha, j}\right\rangle-\left\langle\mathfrak{m}_{\alpha, j}, \mathfrak{m}_{\alpha, j}\right\rangle
$$

Em alguns contextos, será mais conveniente escrever $F_{n, j}$ como acima ao invés de (4.2.72).

Com a notação acima, reescrevemos (4.2.71) como

$$
S_{m, \alpha}^{\star}(s) \leq M^{m} \exp \left\{N\left[\phi_{k_{s}}\left(\mathfrak{u}_{k_{s}}\right)+F_{n, k_{s}}(\beta, \alpha)\right]\right\}, \quad s=0, \ldots, m-1 .
$$

Estimativa para $S_{m, \alpha}^{\star}(m)$. Agora vamos considerar o caso $s=m$, ou seja, o termo

$$
S_{m, \alpha}^{\star}(m)=\sum_{\mathbb{I}_{m, \alpha}^{\star}(m)} e^{\frac{N}{2}\left[\alpha \bar{P}_{k_{m}}+\left\langle 2 \mathfrak{m}^{*}, \underline{x}\right\rangle\right]} .
$$

Neste caso, como $\underline{x} \in \Psi$ é tal que $\underline{x}_{i}=0, \forall i \neq k_{1}, \ldots, k_{m}$, temos que

$$
\left\langle\mathfrak{m}^{*}, \underline{x}\right\rangle=\left\langle\Pi_{0}^{k_{m}} \mathfrak{m}^{*}, \Pi_{0}^{k_{m}} \underline{x}\right\rangle=\phi_{k_{m}}\left(\Pi_{0}^{k_{m}} \underline{x}\right) \stackrel{(4.2 .64)}{\leq} \phi_{k_{m}}\left(\mathfrak{u}_{k_{m}}\right),
$$


e portanto,

$$
S_{m, \alpha}^{\star}(m) \leq M^{m} \exp \left\{\frac{N}{2}\left[2 \phi_{k_{m}}\left(\mathfrak{u}_{k_{m}}\right)+\alpha \bar{P}_{k_{m}}\right]\right\}
$$

O lema seguinte lida com o termo $\alpha \bar{P}_{k_{m}}$.

Lema 4.2.2. Para todo $0 \leq \alpha \leq 1$ e todo $j \in\{0, \ldots, n-1\}$, vale

$$
\alpha \bar{P}_{j} \leq 2 F_{n, j}(\beta, \alpha)
$$

Demonstração. Com efeito, para $\alpha=0$ é imediato. Vamos então supor que $0<\alpha \leq 1$. Com a notação em (4.2.73), temos que

$$
\begin{aligned}
2 F_{n, j}(\beta, \alpha)-\alpha \bar{P}_{j} & =2\left\langle\mathfrak{m}_{\alpha, j}, \Pi_{j}^{n} \mathfrak{m}^{*}\right\rangle-\left\langle\mathfrak{m}_{\alpha, j}, \mathfrak{m}_{\alpha, j}\right\rangle \\
& =\underbrace{\left\langle\mathfrak{m}_{\alpha, j}, \Pi_{j}^{n} \mathfrak{m}^{*}\right\rangle}_{\geq 0}+\left\langle\mathfrak{m}_{\alpha, j}, \Pi_{j}^{n} \mathfrak{m}^{*}\right\rangle-\left\langle\mathfrak{m}_{\alpha, j}, \mathfrak{m}_{\alpha, j}\right\rangle \\
& \geq\left\langle\mathfrak{m}_{\alpha, j}, \Pi_{j}^{n} \mathfrak{m}^{*}-\mathfrak{m}_{\alpha, j}\right\rangle .
\end{aligned}
$$

Agora considere a forma linear

$$
\begin{aligned}
\mathcal{L}_{j}: \Psi_{j}^{n}(\alpha) & \rightarrow \mathbb{R} \\
x & \mapsto \mathcal{L}_{j}(x) \stackrel{\text { def }}{=}\left\langle x-\mathfrak{m}_{\alpha, j}, \Pi_{j}^{n} \mathfrak{m}^{*}-\mathfrak{m}_{\alpha, j}\right\rangle
\end{aligned}
$$

e note que

$$
\left\langle\mathfrak{m}_{\alpha, j}, \Pi_{j}^{n} \mathfrak{m}^{*}-\mathfrak{m}_{\alpha, j}\right\rangle=-\mathcal{L}_{j}(0)
$$

Logo, se $\mathcal{L}_{j}(0) \leq 0$, segue que $\left\langle\mathfrak{m}_{\alpha, j}, \Pi_{j}^{n} \mathfrak{m}^{*}-\mathfrak{m}_{\alpha, j}\right\rangle \geq 0$ e, por fim, (4.2.78) vale. Agora, como $\Psi_{j}^{n}(\alpha)$ é convexo e compacto, portanto, fechado, pelo Teorema A.0.1, uma condição necessária e suficiente para existência do ponto $\mathfrak{m}_{\alpha, j}$ é que

$$
\mathcal{L}_{j}(x) \leq 0
$$

para todo $x \in \Psi_{j}^{n}(\alpha)$. Como $0 \in \Psi_{j}^{n}(\alpha)$, a demonstração está encerrada.

Usando o lema que acabamos de provar, obtemos que

$$
S_{m, \alpha}^{\star}(m) \leq M^{m} \exp \left\{N\left[\phi_{k_{m}}\left(\mathfrak{u}_{k_{m}}\right)+F_{n, k_{m}}(\beta, \alpha)\right]\right\}
$$


Conclusão da Estimativa para $S_{m, \alpha}^{\star}(s)$. Portanto, de (4.2.74) e (4.2.83), segue que

$$
S_{m, \alpha}^{\star}(s) \leq M^{m} \exp \left\{N\left[\phi_{k_{s}}\left(\mathfrak{u}_{k_{s}}\right)+F_{n, k_{s}}(\beta, \alpha)\right]\right\}, \quad \forall s=0, \ldots, m .
$$

Voltando para (4.2.51), concluímos finalmente que

$$
\begin{aligned}
(4.2 .51) & \stackrel{(4.2 .54)}{\leq} \sum_{s=0}^{m}\left(4 c_{u} N\right)^{m} S_{m, \alpha}^{\star}(s) \\
& \stackrel{(4.2 .84)}{\leq}\left(4 c_{u} M N\right)^{m} \sum_{s=0}^{m} \exp \left\{N\left[\phi_{k_{s}}\left(\mathfrak{u}_{k_{s}}\right)+F_{n, k_{s}}(\beta, \alpha)\right]\right\} \\
& \leq(m+1)\left(4 c_{u} M N\right)^{m} \exp \left\{N \bigvee_{s=0}^{m}\left[\phi_{k_{s}}\left(\mathfrak{u}_{k_{s}}\right)+F_{n, k_{s}}(\beta, \alpha)\right]\right\} \\
& \leq(m+1)\left(4 c_{u} M N\right)^{m} \exp \left\{N \bigvee_{j=0}^{n}\left[\phi_{j}\left(\mathfrak{u}_{j}\right)+F_{n, j}(\beta, \alpha)\right]\right\},
\end{aligned}
$$

para todo $N$ grande o suficiente, em que convencionamos $\phi_{0} \equiv F_{n, n} \equiv 0$.

\subsubsection{Conclusão da Estimativa para (4.1.34)}

Nesta subseção concluiremos a estimativa do termo $S_{1}$ e estimaremos os termos $S_{0}$ e $S^{*}$. Por consequência, obteremos a estimativa de $Z_{1, l}^{(1)}\left(\boldsymbol{\xi}, \boldsymbol{\zeta}^{\prime}\right)$. Usando um argumento por complementação obteremos também a estimativa para $Z_{l, \mathrm{~B}}^{(1)}\left(\boldsymbol{\zeta}, \boldsymbol{\xi}^{\prime}\right)$. Encerraremos a subseção com o argumento para a cota superior de (4.2.1).

Começaremos reunindo os resultados das Subseções 4.2.3 e 4.2.4 na seguinte afirmação: do Corolário 4.2 .1 e (4.2.85), para todo $\varepsilon>0$ e todo $N$ grande o suficiente, com $\mathbb{P}$-probabilidade $\geq 1-e^{-\varepsilon N}$, obtemos que

$$
\begin{aligned}
S_{1} & \leq e^{n \beta \beta_{\star} \delta_{0} N} \sum_{m=1}^{n} \sum_{\left[k_{1}, \ldots, k_{m}\right]}(m+1)\left(4 c_{u} M N\right)^{m} \exp \left\{N \bigvee_{j=0}^{n}\left[\phi_{j}\left(\mathfrak{u}_{j}\right)+F_{n, j}(\beta, \alpha)\right]\right\} \\
& \leq(n+1)\left(4 c_{u} M N\right)^{n} e^{n \beta \beta_{\star} \delta_{0} N} \exp \left\{N \bigvee_{j=0}^{n}\left[\phi_{j}\left(\mathfrak{u}_{j}\right)+F_{n, j}(\beta, \alpha)\right]\right\}
\end{aligned}
$$

Isto conclui a estimativa do termo $S_{1}$.

Estimativa para $S_{0}$. Considere agora o termo

$$
S_{0}=\sum_{\boldsymbol{\eta} \in \Omega_{\mathcal{V}^{1}, l-1}} \mathbb{1}\left\{-X_{\boldsymbol{\varphi}_{l}\left(\boldsymbol{\eta}, \boldsymbol{\xi}, \boldsymbol{\zeta}^{\prime}\right)} \in \sqrt{N} \Delta_{0, \ldots, 0}\right\} e^{\left\langle\mathfrak{m}^{*}, \tilde{x}\right\rangle N}
$$

Voltando em (4.2.6), não é difícil concluir que $\left\langle\mathfrak{m}^{*}, \tilde{x}\right\rangle \leq n \beta \beta_{\star} \delta_{0}$. Então, como $V \leq N$, é imediato que

$$
S_{0} \leq e^{n \beta \beta_{\star} \delta_{0} N} 2^{\left|\mathcal{V}^{1, l-1}\right|} \stackrel{(4.2 .18)}{\leq} e^{n \beta \beta_{\star} \delta_{0} N} e^{\frac{1}{2} \alpha \beta_{\star}^{2} N} \leq e^{n \beta \beta_{\star} \delta_{0} N} \exp \left\{N F_{n, 0}(\beta, \alpha)\right\},
$$

em que a última desigualdade segue do Lema 4.2 .2 configurado com $j=0$. 
Estimativa para $S^{*}$. Finalmente, vamos considerar

$$
S^{*}=e^{-\beta H(\overline{\boldsymbol{\sigma}})} \sum_{j_{1}, \ldots, j_{n} \in \mathcal{X}^{*}}\left(\sum_{\boldsymbol{\sigma} \in \Omega_{\mathcal{V}}} \mathbb{1}\left\{-X_{\boldsymbol{\sigma}} \in \sqrt{N} \Delta_{j_{1}, \ldots, j_{n}}\right\}\right) .
$$

A estratégia é provar que, com $\mathbb{P}$-probabilidade $1, S^{*}=0$ para todo $N$ grande o suficiente.

Com efeito, seja $j_{1}, \ldots, j_{n} \in \mathcal{X}^{*}$. Isto significa que em $\Delta_{j_{1}, \ldots, j_{n}}=\Delta_{j_{1}}^{1} \times \cdots \times \Delta_{j_{n}}^{n}$ existe ao menos um $\Delta_{j_{k}}^{k}=\left(\left(1+\delta_{0}\right) \sqrt{P_{k}}, \infty\right)$. Neste caso, se considerarmos o conjunto $\mathcal{A}_{n}(N, \delta)$ definido em (2.1.31) configurado $\operatorname{com} \delta=\delta_{0}$, como $V_{j} \leq p_{j} N$, não é difícil concluir que

$$
\begin{aligned}
\sum_{j_{1}, \ldots, j_{n} \in \mathcal{X}^{*}} \mathbb{1}\left\{-X_{\boldsymbol{\sigma}} \in \sqrt{N} \Delta_{j_{1}, \ldots, j_{n}}\right\} & =\mathbb{1}\left\{-X_{\boldsymbol{\sigma}} \in \bigcup_{j_{1}, \ldots, j_{n} \in \mathcal{X}^{*}} \sqrt{N} \Delta_{j_{1}, \ldots, j_{n}}\right\} \\
& \leq \mathbb{1}\left\{-X_{\boldsymbol{\sigma}} \in \mathcal{A}_{n}^{c}\left(N, \delta_{0}\right)\right\} .
\end{aligned}
$$

Consequentemente,

$$
\begin{aligned}
\mathbb{P}\left[\sum_{j_{1}, \ldots, j_{n} \in \mathcal{X}^{*}} \sum_{\boldsymbol{\sigma} \in \Omega_{\mathcal{V}}}\right. & \left.\mathbb{1}\left\{-X_{\boldsymbol{\sigma}} \in \sqrt{N} \Delta_{j_{1}, \ldots, j_{n}}\right\} \geq \frac{1}{2}\right] \\
& \leq \mathbb{P}\left[\sum_{\boldsymbol{\sigma} \in \Omega_{\mathcal{V}}} \mathbb{1}\left\{-X_{\boldsymbol{\sigma}} \in \mathcal{A}_{n}^{c}\left(N, \delta_{0}\right)\right\} \geq \frac{1}{2}\right] \\
& =\mathbb{P}\left[\mathcal{A}_{n}^{c}\left(N, \delta_{0}\right)\right] .
\end{aligned}
$$

Invocando a Proposição 2.1.1, com $\mathbb{P}$-probabilidade 1,

$$
0 \leq \sum_{j_{1}, \ldots, j_{n} \in \mathcal{X}^{*}} \sum_{\boldsymbol{\sigma} \in \Omega_{\mathcal{V}}} \mathbb{1}\left\{-X_{\boldsymbol{\sigma}} \in \sqrt{N} \Delta_{j_{1}, \ldots, j_{n}}\right\}<\frac{1}{2}
$$

para todo $N$ grande o suficiente.

Como a soma em (4.2.92) só pode assumir valores inteiros, então ela é necessariamente igual a zero para todo $N$ grande o suficiente. Este fato é suficiente para concluirmos que

$$
S^{*}=0,
$$

$\mathbb{P}$-quase certamente.

Reunindo (4.2.86), (4.2.88) e (4.2.93), concluímos então que, para todo $\varepsilon>0$, com $\mathbb{P}$ probabilidade $\geq 1-e^{-\varepsilon N}$,

$$
\begin{aligned}
& \max _{\boldsymbol{\xi} \in \Omega_{\mathcal{V} l, l} \max _{\boldsymbol{\zeta}^{\prime} \in \Omega_{\mathcal{V}}}} Z_{1, l}^{(1)}\left(\boldsymbol{\xi}, \boldsymbol{\zeta}^{\prime}\right) \\
& \leq e^{n \beta \beta_{\star} \delta_{0} N}\left(\exp \left\{N F_{n, 0}(\beta, \alpha)\right\}+(n+1)\left(4 c_{u} M N\right)^{n} \exp \left\{N \bigvee_{j=0}^{n}\left[\phi_{j}\left(\mathfrak{u}_{j}\right)+F_{n, j}(\beta, \alpha)\right]\right\}\right) \\
& \leq C(\beta, n, M, N) \exp \left\{N \bigvee_{j=0}^{n} G_{j}(\beta, \alpha)\right\},
\end{aligned}
$$

para todo $N$ grande o suficiente, em que estamos denotando

$$
G_{j}(\beta, \alpha) \stackrel{\text { def }}{=} \phi_{j}\left(\mathfrak{u}_{j}\right)+F_{n, j}(\beta, \alpha),
$$




$$
C(\beta, n, M, N) \stackrel{\text { def }}{=} 2(n+1)\left(4 c_{u} M N e^{\beta \beta_{\star} \delta_{0} N}\right)^{n}
$$

Estimativa para $Z_{l, \mathrm{~B}}^{(1)}\left(\boldsymbol{\zeta}, \boldsymbol{\xi}^{\prime}\right)$. Foi assumido em (4.2.18) que $l-1=\alpha \mathrm{B}$, para algum $0<\alpha<1$. Então, por (4.1.15), obtemos que

$$
\left|\mathcal{V}^{l+1, \mathrm{~B}}\right|=(\mathrm{B}-l) \sum_{j=1}^{n} \mathrm{v}_{j}=(\mathrm{B}-\alpha \mathrm{B}-1) \sum_{j=1}^{n} \mathrm{v}_{j} \stackrel{(2.1 .12)}{\leq} \bar{\alpha} V,
$$

em que

$$
\bar{\alpha}=1-\alpha .
$$

Logo, o Corolário 4.2.1 vale com $\alpha$ substituído por $\bar{\alpha}$, e portanto, usando uma sequência de argumentos análogos aos das Subseções 4.2.3, 4.2.4 e 4.2.5, obtemos a seguinte afirmação: para todo $\varepsilon>0$, com $\mathbb{P}$-probabilidade $\geq 1-e^{-\varepsilon N}$,

$$
\max _{\boldsymbol{\xi}^{\prime} \in \Omega_{\mathcal{V} l, l}} \max _{\boldsymbol{\zeta} \in \Omega_{\mathcal{V}}} Z_{l, \mathrm{~B}}^{(1)}\left(\boldsymbol{\zeta}, \boldsymbol{\xi}^{\prime}\right) \leq C(\beta, n, M, N) \exp \left\{N \bigvee_{j=0}^{n} G_{j}(\beta, \bar{\alpha})\right\},
$$

para todo $N$ grande o suficiente.

Conclusão da Estimativa para (4.2.1). Juntando (4.2.94) e (4.2.99), segue que para todo $\varepsilon>0$, com $\mathbb{P}$-probabilidade $\geq 1-e^{-\varepsilon N}$,

$$
\begin{aligned}
(4.2 .1) & \leq C^{2}(\beta, n, M, N) \max _{0 \leq \alpha \leq 1} \exp \left\{N\left(\bigvee_{j=0}^{n} G_{j}(\beta, \alpha)+\bigvee_{k=0}^{n} G_{k}(\beta, \bar{\alpha})\right)\right\} \\
& =C^{2}(\beta, n, M, N) \exp \left\{N \max _{0 \leq \alpha \leq 1}\left\{\bigvee_{j=0}^{n} G_{j}(\beta, \alpha)+\bigvee_{k=0}^{n} G_{k}(\beta, \bar{\alpha})\right\}\right\},
\end{aligned}
$$

para todo $N$ grande o suficiente.

Observação 4.2.4. É importante notar que a desigualdade em (4.2.100) não é trivial no sentido que o termo do máximo no lado direito é finito. Isso é verdade porque, para todo $\beta>0$ fixado, a função dentro do sinal de $\max _{0 \leq \alpha \leq 1}$ é uma composição de funções claramente contínuas com respeito a $\alpha$, e como o intervalo [0,1] é compacto, o Teorema A.0.2 garante que ela é limitada.

Note que temos resumido todo a questão de estimar o termo (4.2.1) no seguinte problema de otimização:

$$
\max _{0 \leq \alpha \leq 1}\left\{\bigvee_{j=0}^{n} G_{j}(\beta, \alpha)+\bigvee_{k=0}^{n} G_{k}(\beta, \bar{\alpha})\right\}
$$

para todos os valores de $\beta>0$. Apesar do que parece, note que estamos diante de um problema completamente determinístico, o que, em certo sentido, facilita sua análise. Em [FIKP98] um problema análogo foi resolvido. Nesse caso (lembre que $n=1$ lá), Fontes et al. provaram que

$$
\max _{0 \leq \alpha \leq 1}\left\{\bigvee_{j=0}^{1} G_{j}(\beta, \alpha)+\bigvee_{k=0}^{1} G_{k}(\beta, \bar{\alpha})\right\} \leq F_{1}(\beta)+\beta \beta_{1}
$$


qualquer que seja o valor de $\beta>0$. É importante enfatizar que a parcela $\beta \beta_{1}$, linear em $\beta$, é também a energia livre do REM sob o regime de baixas temperaturas. Portanto, é razoável esperar que no caso geral tenhamos

$$
(4.2 .101) \leq F_{n}(\beta)+\beta \sum_{\ell=1}^{\ell_{n}} \beta_{\ell} \sum_{k=J_{\ell-1}^{*}+1}^{J_{\ell}^{*}} a_{k}, \quad \forall \beta>0 .
$$

De fato, é isso que acontece, como provamos na seguinte proposição.

Proposição 4.2.2. Para todo $\beta>0$,

$$
\max _{0 \leq \alpha \leq 1}\left\{\bigvee_{j=0}^{n} G_{j}(\beta, \alpha)+\bigvee_{k=0}^{n} G_{k}(\beta, \bar{\alpha})\right\} \leq F_{n}(\beta)+\beta \sum_{\ell=1}^{\ell_{n}} \beta_{\ell} \sum_{k=J_{\ell-1}^{*}+1}^{J_{\ell}^{*}} a_{k}
$$

Demonstração. Subseção 4.2.6.

Agora note que para todo $\beta>0$, todo $\varepsilon>0$ e para todo $N$ grande o suficiente

$$
\begin{aligned}
\frac{1}{N} \log C^{2}(\beta, n, M, N) & =\frac{2}{N} \log \left(2(n+1)\left(4 c_{u} M N e^{\beta \beta_{\star} \delta_{0} N}\right)^{n}\right) \\
& =\frac{2}{N} \log 2(n+1)+\frac{2 n}{N} \log \left(4 c_{u} M N\right)+2 n \beta \beta_{\star} \delta_{0} \\
& \leq \frac{\varepsilon}{3}+2 n \beta \beta_{\star} \delta_{0} .
\end{aligned}
$$

Este fato junto com a Proposição 4.2.2 implicam que

$$
\begin{aligned}
\frac{1}{N} \log & \left(C^{2}(\beta, n, M, N) \exp \left\{N \max _{0 \leq \alpha \leq 1}\left\{\bigvee_{j=0}^{n} G_{j}(\beta, \alpha)+\bigvee_{k=0}^{n} G_{k}(\beta, \bar{\alpha})\right\}\right\}\right) \\
\leq & F_{n}(\beta)+\beta \sum_{\ell=1}^{\ell_{n}} \beta_{\ell} \sum_{k=J_{\ell-1}^{*}+1}^{J_{\ell}^{*}} a_{k}+\frac{2 \varepsilon}{3},
\end{aligned}
$$

para todo $\beta>0$, todo $\varepsilon>0$ e todo $N$ grande o suficiente se escolhermos $M>\frac{4 n \beta \beta_{\star}}{\varepsilon}$ em (4.2.3).

Portanto, voltando para (4.2.1), de (4.2.100) e (4.2.106), para todo $\beta>0$ e todo $\varepsilon>0$, com $\mathbb{P}$-probabilidade $\geq 1-e^{-\varepsilon N}$,

$$
\frac{1}{N} \log \left(4^{\mathcal{S}}(4.2 .1)\right) \leq F_{n}(\beta)+\beta \sum_{\ell=1}^{\ell_{n}} \beta_{\ell} \sum_{k=J_{\ell-1}^{*}+1}^{J_{\ell}^{*}} a_{k}+\varepsilon,
$$

para todo $N$ grande o suficiente, em que usamos também que $\mathcal{S}=o(N)$.

\subsubsection{Prova da Proposição 4.2.2}

As ferramentas fundamentais usadas nesta subseção são o Teorema da Projeção sobre um Convexo Fechado A.0.1 e a desigualdade de Cauchy-Schwarz. Usaremos também sistematicamente o fato que, para todo $\beta>0$ fixado, as funções de $\alpha$ são limitadas. Isso segue como consequência do Teorema A.0.2. 
Para começar, fixados $\beta>0$ e $0 \leq \alpha \leq 1$, não é difícil ver que

$$
\bigvee_{j=0}^{n} G_{j}(\beta, \alpha)+\bigvee_{k=0}^{n} G_{k}(\beta, \bar{\alpha}) \leq \bigvee_{j=0}^{n} \bigvee_{k=0}^{n}\left[G_{j}(\beta, \alpha)+G_{k}(\beta, \bar{\alpha})\right]
$$

Consequentemente, e também usando simetria, temos que

$$
\begin{aligned}
(4.2 .101) & \leq \bigvee_{j=0}^{n} \bigvee_{k=1}^{n} \max _{0 \leq \alpha \leq 1}\left\{G_{j}(\beta, \alpha)+G_{k}(\beta, \bar{\alpha})\right\} \\
& =\bigvee_{j=0}^{n} \bigvee_{k=j}^{n} \max _{0 \leq \alpha \leq 1}\left\{G_{j}(\beta, \alpha)+G_{k}(\beta, \bar{\alpha})\right\} \\
& \stackrel{(4.2 .95)}{=} \bigvee_{j=0}^{n} \bigvee_{k=j}^{n} \max _{0 \leq \alpha \leq 1}\left\{\phi_{j}\left(\mathfrak{u}_{j}\right)+\phi_{k}\left(\mathfrak{u}_{k}\right)+F_{n, j}(\beta, \alpha)+F_{n, k}(\beta, \bar{\alpha})\right\} \\
& \leq \bigvee_{j=0}^{n} \bigvee_{k=j}^{n}\left[\phi_{j}\left(\mathfrak{u}_{j}\right)+\phi_{k}\left(\mathfrak{u}_{k}\right)+\max _{0 \leq \alpha \leq 1}\left\{F_{n, j}(\beta, \alpha)+F_{n, k}(\beta, \bar{\alpha})\right\}\right] \\
& =M_{1} \vee M_{2} \vee\left[2 \phi_{n}\left(\mathfrak{u}_{n}\right)\right],
\end{aligned}
$$

em que a última desigualdade segue do Lema A.0.2 e estamos denotando

$$
M_{1} \stackrel{\text { def }}{=} \bigvee_{j=0}^{n-1} \bigvee_{k=j}^{n-1}\left[\phi_{j}\left(\mathfrak{u}_{j}\right)+\phi_{k}\left(\mathfrak{u}_{k}\right)+\max _{0 \leq \alpha \leq 1}\left\{F_{n, j}(\beta, \alpha)+F_{n, k}(\beta, \bar{\alpha})\right\}\right]
$$

e

$$
M_{2} \stackrel{\text { def }}{=} \bigvee_{j=0}^{n-1}\left[\phi_{n}\left(\mathfrak{u}_{n}\right)+\phi_{j}\left(\mathfrak{u}_{j}\right)+\max _{0 \leq \alpha \leq 1}\left\{F_{n, j}(\beta, \alpha)\right\}\right]
$$

O próximo passo será uma consequência direta do seguinte lema.

Lema 4.2.3. Para todo $0 \leq j \leq k \leq n-1$ e todo $\beta>0$ vale,

(i) $\max _{0 \leq \alpha \leq 1}\left\{F_{n, j}(\beta, \alpha)+F_{n, k}(\beta, \bar{\alpha})\right\} \leq F_{n, j}(\beta)+\frac{1}{2}\left\|\Pi_{k}^{n} \mathfrak{m}^{*}\right\|^{2}-\frac{1}{2}\left\|\Pi_{k}^{n} \mathfrak{m}^{*}-\mathfrak{w}_{1, k}\right\|^{2}$.

(ii) $\max _{0 \leq \alpha \leq 1}\left\{F_{n, j}(\beta, \alpha)\right\} \leq F_{n, j}(\beta)$, em que $F_{n, j}(\beta)=F_{n, j}(\beta, 1)$.

(iii) $\phi_{j}\left(\mathfrak{u}_{j}\right)+\frac{1}{2}\left\|\Pi_{j}^{n} \mathfrak{m}^{*}\right\|^{2}-\frac{1}{2}\left\|\Pi_{j}^{n} \mathfrak{m}^{*}-\mathfrak{w}_{1, j}\right\|^{2} \leq \phi_{n}\left(\mathfrak{u}_{n}\right)$.

(iv) $\phi_{j}\left(\mathfrak{u}_{j}\right)+F_{n, j}(\beta) \leq F_{n}(\beta)$.

Demonstração. (i) Com a notação (4.2.72), escrevemos

$$
\begin{aligned}
& 2\left(F_{n, j}(\beta, \alpha)+F_{n, k}(\beta, \bar{\alpha})\right) \\
& \quad=\alpha \bar{P}_{j}+\bar{\alpha} \bar{P}_{k}+\left\|\Pi_{j}^{n} \mathfrak{m}^{*}\right\|^{2}+\left\|\Pi_{k}^{n} \mathfrak{m}^{*}\right\|^{2}-\left\|\Pi_{j}^{n} \mathfrak{m}^{*}-\mathfrak{m}_{\alpha, j}\right\|^{2}-\left\|\Pi_{k}^{n} \mathfrak{m}^{*}-\mathfrak{m}_{\bar{\alpha}, k}\right\|^{2},
\end{aligned}
$$

e aplicando o máximo sobre $0 \leq \alpha \leq 1$, segue do Lema A.0.2 que

$$
\begin{aligned}
& 2 \max _{0 \leq \alpha \leq 1}\left\{F_{n, j}(\beta, \alpha)+F_{n, k}(\beta, \bar{\alpha})\right\} \\
& \leq \max _{0 \leq \alpha \leq 1}\left\{\alpha \bar{P}_{j}+\bar{\alpha} \bar{P}_{k}\right\}+\left\|\Pi_{j}^{n} \mathfrak{m}^{*}\right\|^{2}+\left\|\Pi_{k}^{n} \mathfrak{m}^{*}\right\|^{2}-\min _{0 \leq \alpha \leq 1}\left\{\left\|\Pi_{j}^{n} \mathfrak{m}^{*}-\mathfrak{m}_{\alpha, j}\right\|^{2}\right\} \\
& \quad-\min _{0 \leq \alpha \leq 1}\left\{\left\|\Pi_{k}^{n} \mathfrak{m}^{*}-\mathfrak{m}_{\bar{\alpha}, k}\right\|^{2}\right\} .
\end{aligned}
$$


Agora, observe que: (1)

$$
\max _{0 \leq \alpha \leq 1}\left\{\alpha \bar{P}_{j}+\bar{\alpha} \bar{P}_{k}\right\} \stackrel{(4.2 .25)}{=} \max _{0 \leq \alpha \leq 1}\left\{\alpha \beta_{\star}^{2}\left(p_{j+1}+\cdots+p_{k}\right) \mathbb{1}_{\{j<k\}}+\bar{P}_{k}\right\} \leq \bar{P}_{j}
$$

e (2), pelo Teorema A.0.1, denotando $\Psi_{i}^{n} \equiv \Psi_{i}^{n}(1)$ para $i \in\{0, \ldots, n-1\}$,

$$
\begin{aligned}
\min _{0 \leq \alpha \leq 1}\left\|\Pi_{j}^{n} \mathfrak{m}^{*}-\mathfrak{w}_{\alpha, j}\right\|^{2}=\left(\min _{0 \leq \alpha \leq 1} \mathrm{~d}\left(\Pi_{j}^{n} \mathfrak{m}^{*}, \Psi_{j}^{n}(\alpha)\right)\right)^{2} & =\left(\mathrm{d}\left(\Pi_{j}^{n} \mathfrak{m}^{*}, \Psi_{j}^{n}\right)\right)^{2} \\
& =\left\|\Pi_{j}^{n} \mathfrak{m}^{*}-\mathfrak{w}_{1, j}\right\|^{2}
\end{aligned}
$$

e

$$
\begin{aligned}
\min _{0 \leq \alpha \leq 1}\left\|\Pi_{k}^{n} \mathfrak{m}^{*}-\mathfrak{w}_{\bar{\alpha}, k}\right\|^{2}=\left(\min _{0 \leq \alpha \leq 1} \mathrm{~d}\left(\Pi_{k}^{n} \mathfrak{m}^{*}, \Psi_{k}^{n}(\bar{\alpha})\right)\right)^{2} & =\left(\mathrm{d}\left(\Pi_{k}^{n} \mathfrak{m}^{*}, \Psi_{k}^{n}\right)\right)^{2} \\
& =\left\|\Pi_{k}^{n} \mathfrak{m}^{*}-\mathfrak{w}_{1, k}\right\|^{2}
\end{aligned}
$$

Portanto, de (4.2.114), (4.2.115) e (4.2.116) obtemos que

$$
\begin{aligned}
2 \max _{0 \leq \alpha \leq 1} & \left\{F_{n, j}(\beta, \alpha)+F_{n, k}(\beta, \bar{\alpha})\right\} \\
& \leq \bar{P}_{j}+\left\|\Pi_{j}^{n} \mathfrak{m}^{*}\right\|^{2}+\left\|\Pi_{k}^{n} \mathfrak{m}^{*}\right\|^{2}-\left\|\Pi_{j}^{n} \mathfrak{m}^{*}-\mathfrak{w}_{1, j}\right\|^{2}-\left\|\Pi_{k}^{n} \mathfrak{m}^{*}-\mathfrak{w}_{1, k}\right\|^{2} \\
& =2 F_{n, j}(\beta)+\left\|\Pi_{k}^{n} \mathfrak{m}^{*}\right\|^{2}-\left\|\Pi_{k}^{n} \mathfrak{m}^{*}-\mathfrak{w}_{1, k}\right\|^{2}
\end{aligned}
$$

(ii) Com argumentos usados no item anterior, obtemos que

$$
\begin{aligned}
2 \max _{0 \leq \alpha \leq 1}\left\{F_{n, j}(\beta, \alpha)\right\} & \leq \max _{0 \leq \alpha \leq 1}\left\{\alpha \bar{P}_{j}\right\}+\left\|\Pi_{j}^{n} \mathfrak{m}^{*}\right\|^{2}-\min _{0 \leq \alpha \leq 1}\left\{\left\|\Pi_{j}^{n} \mathfrak{m}^{*}-\mathfrak{m}_{\alpha, j}\right\|^{2}\right\} \\
& \leq 2 F_{n, j}(\beta)
\end{aligned}
$$

Para provar as duas últimas desigualdades vamos dividir em dois casos: $j=0$ e $j=1, \ldots, n-$ 1.

(iii) Por definição $\|x\|^{2}=\langle x, x\rangle$. Neste caso, usando as propriedades de produto interno, obtemos que

$$
\begin{aligned}
\left\|\Pi_{j}^{n} \mathfrak{m}^{*}\right\|^{2}-\left\|\Pi_{j}^{n} \mathfrak{m}^{*}-\mathfrak{w}_{1, j}\right\|^{2} & =\left\langle\Pi_{j}^{n} \mathfrak{m}^{*}, \Pi_{j}^{n} \mathfrak{m}^{*}\right\rangle-\left\langle\Pi_{j}^{n} \mathfrak{m}^{*}-\mathfrak{w}_{1, j}, \Pi_{j}^{n} \mathfrak{m}^{*}-\mathfrak{w}_{1, j}\right\rangle \\
& =2\left\langle\mathfrak{w}_{1, j}, \Pi_{j}^{n} \mathfrak{m}^{*}\right\rangle-\underbrace{\left\langle\mathfrak{w}_{1, j}, \mathfrak{w}_{1, j}\right\rangle}_{\geq 0} \\
& \leq 2\left\langle\mathfrak{w}_{1, j}, \Pi_{j}^{n} \mathfrak{m}^{*}\right\rangle .
\end{aligned}
$$

Daí, se $j=1, \ldots, n-1$,

$$
\begin{aligned}
2 \phi_{j}\left(\mathfrak{u}_{j}\right)+\left\|\Pi_{j}^{n} \mathfrak{m}^{*}\right\|^{2}-\left\|\Pi_{j}^{n} \mathfrak{m}^{*}-\mathfrak{w}_{1, j}\right\|^{2} & \leq 2\left\langle\mathfrak{u}_{j}, \Pi_{0}^{j} \mathfrak{m}^{*}\right\rangle+2\left\langle\mathfrak{w}_{1, j}, \Pi_{j}^{n} \mathfrak{m}^{*}\right\rangle \\
& =2\left\langle\mathfrak{u}_{j} * \mathfrak{w}_{1, j}, \mathfrak{m}^{*}\right\rangle
\end{aligned}
$$


em que "*" denota a concatenação de sequências finitas ${ }^{\dagger}$. Como $\mathfrak{u}_{j} \in \Psi_{j}$ e $\mathfrak{w}_{1, j} \in \Psi_{j}^{n}$, temos que $\mathfrak{u}_{j} * \mathfrak{w}_{1, j} \in \Psi$, e portanto,

$$
\left\langle\mathfrak{u}_{j} * \mathfrak{w}_{1, j}, \mathfrak{m}^{*}\right\rangle=\phi_{n}\left(\mathfrak{u}_{j} * \mathfrak{w}_{1, j}\right) \leq \phi_{n}\left(\mathfrak{u}_{n}\right)
$$

assim concluindo que:

$$
2 \phi_{j}\left(\mathfrak{u}_{j}\right)+\left\|\Pi_{j}^{n} \mathfrak{m}^{*}\right\|^{2}-\left\|\Pi_{j}^{n} \mathfrak{m}^{*}-\mathfrak{w}_{1, j}\right\|^{2} \leq 2 \phi_{n}\left(\mathfrak{u}_{n}\right) .
$$

Se $j=0$, então $\phi_{0} \equiv 0$ e como $\mathfrak{w}_{1,0} \in \Psi_{0}^{n}=\Psi$, segue que

$$
\left\|\Pi_{0}^{n} \mathfrak{m}^{*}\right\|^{2}-\left\|\Pi_{0}^{n} \mathfrak{m}^{*}-\mathfrak{w}_{1,0}\right\|^{2} \leq 2\left\langle\mathfrak{w}_{1,0}, \mathfrak{m}^{*}\right\rangle=2 \phi_{n}\left(\mathfrak{w}_{1,0}\right) \leq 2 \phi_{n}\left(\mathfrak{u}_{n}\right)
$$

(iv) O caso mais simples, $j=0$, segue da convenção $\phi_{0} \equiv 0$ e do fato que $F_{n, 0}(\beta)=F_{n}(\beta)$ como pode ser conferido em (4.2.72).

Para o outro caso, $j=1, \ldots, n-1$, com a notação em (4.2.73), escrevemos primeiro que

$$
\begin{aligned}
2\left(\phi_{j}\left(\mathfrak{u}_{j}\right)+F_{n, j}(\beta)\right) & =2\left\langle\mathfrak{u}_{j}, \Pi_{0}^{j} \mathfrak{m}^{*}\right\rangle+\bar{P}_{j}+2\left\langle\mathfrak{w}_{1, j}, \Pi_{j}^{n} \mathfrak{m}^{*}\right\rangle-\left\langle\mathfrak{w}_{1, j}, \mathfrak{w}_{1, j}\right\rangle \\
& =\bar{P}_{j}+2\left\langle\mathfrak{u}_{j} * \mathfrak{w}_{1, j}, \mathfrak{m}^{*}\right\rangle-\left\langle\mathfrak{w}_{1, j}, \mathfrak{w}_{1, j}\right\rangle \\
& =\beta_{\star}^{2}+2\left\langle\mathfrak{u}_{j} * \mathfrak{w}_{1, j}, \mathfrak{m}^{*}\right\rangle-P_{j}-\left\langle\mathfrak{w}_{1, j}, \mathfrak{w}_{1, j}\right\rangle .
\end{aligned}
$$

Agora, usando o fato que $\mathfrak{u}_{j} \in \operatorname{ext} \Psi_{j} \subseteq \partial \Psi_{j}{ }^{\ddagger \ddagger}$, obtemos que

$$
\left\langle\mathfrak{u}_{j}, \mathfrak{u}_{j}\right\rangle=P_{j} .
$$

Substituindo esta última igualdade em (4.2.124), temos que

$$
\begin{aligned}
2\left(\phi_{j}\left(\mathfrak{u}_{j}\right)+F_{n, j}(\beta)\right)= & \beta_{\star}^{2}+2\left\langle\mathfrak{u}_{j} * \mathfrak{w}_{1, j}, \mathfrak{m}^{*}\right\rangle-\left\langle\mathfrak{u}_{j}, \mathfrak{u}_{j}\right\rangle-\left\langle\mathfrak{w}_{1, j}, \mathfrak{w}_{1, j}\right\rangle \\
& =\beta_{\star}^{2}+2\left\langle\mathfrak{u}_{j} * \mathfrak{w}_{1, j}, \mathfrak{m}^{*}\right\rangle-\left\langle\mathfrak{u}_{j} * \mathfrak{w}_{1, j}, \mathfrak{u}_{j} * \mathfrak{w}_{1, j}\right\rangle \\
& =\beta_{\star}^{2}+\left\|\mathfrak{m}^{*}\right\|^{2}-\left\|\mathfrak{u}_{j} * \mathfrak{w}_{1, j}-\mathfrak{m}^{*}\right\|^{2},
\end{aligned}
$$

em que a última igualdade segue das propriedades do produto interno.

Como $\mathfrak{u}_{j} * \mathfrak{w}_{1, j} \in \Psi$, é imediato que

$$
\left\|\mathfrak{m}^{*}-\mathfrak{u}_{j} * \mathfrak{w}_{1, j}\right\|^{2} \geq\left\|\mathfrak{m}^{*}-\mathfrak{m}\right\|^{2}=\left(\mathrm{d}\left(\mathfrak{m}^{*}, \Psi\right)\right)^{2},
$$

${ }^{\dagger}$ Dadas duas sequências finitas $x=\left(x_{i}\right)_{i=1}^{n}$ e $y=\left(y_{i}\right)_{i=1}^{m}$, a concatenação de $x$ e $y$, denotado por $x * y$, é definida por

$$
x * y \stackrel{\text { def }}{=}\left(x_{1}, \ldots, x_{n}, y_{1}, \ldots, y_{m}\right) .
$$

\footnotetext{
${ }^{\ddagger \ddagger}$ Este fato segue da convexidade do conjunto $\Psi_{j}$.
} 
e portanto,

$$
2\left(\phi_{j}\left(\mathfrak{u}_{j}\right)+F_{n, j}(\beta)\right) \leq \beta_{\star}^{2}+\left\|\mathfrak{m}^{*}\right\|^{2}-\left\|\mathfrak{m}^{*}-\mathfrak{m}\right\|^{2}=2 F_{n}(\beta),
$$

encerrando a demonstração.

Como consequência do Lema 4.2.3, temos o seguinte:

$$
\begin{aligned}
M_{1} & \left.\stackrel{(i)}{\leq} \bigvee_{j=0}^{n-1} \bigvee_{k=j}^{n-1}\left[\phi_{j}\left(\mathfrak{u}_{j}\right)+\phi_{k}\left(\mathfrak{u}_{k}\right)+F_{n, j}(\beta)+\frac{1}{2}\left\|\Pi_{k}^{n} \mathfrak{m}^{*}\right\|^{2}-\frac{1}{2}\left\|\Pi_{k}^{n} \mathfrak{m}^{*}-\mathfrak{w}_{1, k}\right\|^{2}\right\}\right] \\
& \stackrel{\text { (iii) })}{\mathrm{e}} \leq{ }^{(i v)} \bigvee_{j=0}^{n-1} \bigvee_{k=j}^{n-1}\left[F_{n}(\beta)+\phi_{n}\left(\mathfrak{u}_{n}\right)\right] \\
& =F_{n}(\beta)+\phi_{n}\left(\mathfrak{u}_{n}\right),
\end{aligned}
$$

$$
\begin{aligned}
M_{2} & \stackrel{(i i)}{\leq} \bigvee_{j=0}^{n-1}\left[\phi_{n}\left(\mathfrak{u}_{n}\right)+\phi_{j}\left(\mathfrak{u}_{j}\right)+F_{n, j}(\beta)\right] \\
& \stackrel{(i v)}{\leq} \bigvee_{j=0}^{n-1}\left[F_{n}(\beta)+\phi_{n}\left(\mathfrak{u}_{n}\right)\right] \\
& =F_{n}(\beta)+\phi_{n}\left(\mathfrak{u}_{n}\right)
\end{aligned}
$$

e

$$
2 \phi_{n}\left(\mathfrak{u}_{n}\right) \stackrel{(2.1 .56)}{\leq} F_{n}(\beta)+\phi_{n}\left(\mathfrak{u}_{n}\right)
$$

Juntando agora (4.2.109), (4.2.129), (4.2.130) e (4.2.131), obtemos finalmente que

$$
\max _{0 \leq \alpha \leq 1}\left\{\bigvee_{j=0}^{n} G_{j}(\beta, \alpha)+\bigvee_{k=0}^{n} G_{k}(\beta, \bar{\alpha})\right\} \leq F_{n}(\beta)+\phi_{n}\left(\mathfrak{u}_{n}\right), \quad \forall \beta>0
$$

O próximo lema conclui a prova da Proposição 4.2.2.

Lema 4.2.4. Seja $\mathfrak{u}_{n} \in \mathbb{R}^{n}$ dado por

$$
\mathfrak{u}_{n, k} \stackrel{\text { def }}{=} \beta_{\ell} \sqrt{a_{k}}, \text { se } k \in\left\{J_{\ell-1}^{*}+1, \ldots, J_{\ell+1}^{*}\right\} \text { para algum } \ell=1, \ldots, \ell_{n},
$$

Então, $\mathfrak{u}_{n} \in \Psi$ e

$$
\max _{x \in \Psi} \phi_{n}(x)=\phi_{n}\left(\mathfrak{u}_{n}\right)=\beta \sum_{\ell=1}^{\ell_{n}} \beta_{\ell} \sum_{k=J_{\ell-1}^{*}+1}^{J_{\ell}^{*}} a_{k} .
$$

Demonstração. A prova deste lema tem sua inspiração na demonstração do Lema 3.1 em [DD02]. A ferramenta fundamental aqui, assim como foi em [DD02], é a desigualdade de Cauchy-Schwarz. 
O fato que $\mathfrak{u}_{n} \in \Psi$ é consequência imediata da construção das sequências $\left\{\beta_{\ell}\right\}_{\ell=1}^{\ell_{n}}$ e $\left\{J_{\ell}^{*}\right\}_{\ell=1}^{\ell_{n}}$ dadas em (2.1.51) e (2.1.52).

Seja $x \in \Psi$. Dado $\ell \in\left\{1, \ldots, \ell_{n}\right\}$, pela desigualdade de Cauchy-Schwarz, segue que

$$
\left\langle\Pi_{0}^{J_{\ell}^{*}} \mathfrak{u}_{n}, \Pi_{0}^{J_{\ell}^{*}} x\right\rangle \leq\left\|\Pi_{0}^{J_{\ell}^{*}} \mathfrak{u}_{n}\right\| \cdot\left\|\Pi_{0}^{J_{\ell}^{*}} x\right\| \leq\left\|\Pi_{0}^{J_{\ell}^{*}} \mathfrak{u}_{n}\right\| \cdot \sqrt{P_{J_{\ell}^{*}}}
$$

em que a última desigualdade segue de $x$ pertencer ao conjunto $\Psi$.

Agora, note que

$$
\left\|\Pi_{0}^{J_{\ell}^{*}} \mathfrak{u}_{n}\right\|^{2}=\sum_{i=1}^{\ell} \beta_{i}^{2} \sum_{k=J_{i-1}^{*}+1}^{J_{i}^{*}} a_{k}=P_{J_{\ell}^{*}}
$$

Então,

$$
\left\langle\Pi_{0}^{J_{\ell}^{*}} \mathfrak{u}_{n}, \Pi_{0}^{J_{\ell}^{*}} x\right\rangle \leq\left\|\Pi_{0}^{J_{\ell}^{*}} \mathfrak{u}_{n}\right\|^{2}=\left\langle\Pi_{0}^{J_{\ell}^{*}} \mathfrak{u}_{n}, \Pi_{0}^{J_{\ell}^{*}} \mathfrak{u}_{n}\right\rangle
$$

e daí,

$$
\left\langle\Pi_{0}^{J_{\ell}^{*}} \mathfrak{u}_{n}, \Pi_{0}^{J_{\ell}^{*}} \mathfrak{u}_{n}-\Pi_{0}^{J_{\ell}^{*}} x\right\rangle=\sum_{i=1}^{\ell} \sum_{k=J_{i-1}^{*}+1}^{J_{i}^{*}} \beta_{i} \sqrt{a_{k}}\left(\beta_{i} \sqrt{a_{k}}-x_{k}\right) \geq 0,
$$

para todo $\ell \in\left\{1, \ldots, \ell_{n}\right\}$.

Introduzindo os números $\left(\beta \beta_{\ell}^{-1}\right)_{\ell=1}^{\ell_{n}}$ na soma, note que $\beta \beta_{1}^{-1} \geq \cdots \geq \beta \beta_{\ell_{n}}^{-1} \geq 0$, segue do Lema A.0.1 que

$$
\sum_{i=1}^{\ell_{n}} \beta \beta_{i}^{-1} \sum_{k=J_{i-1}^{*}+1}^{J_{i}^{*}} \beta_{i} \sqrt{a_{k}}\left(\beta_{i} \sqrt{a_{k}}-x_{k}\right) \geq 0
$$

Como

$$
\begin{aligned}
\sum_{i=1}^{\ell_{n}} \beta \beta_{i}^{-1} \sum_{k=J_{i-1}^{*}+1}^{J_{i}^{*}} \beta_{i} \sqrt{a_{k}}\left(\beta_{i} \sqrt{a_{k}}-x_{k}\right) & =\sum_{i=1}^{\ell_{n}} \sum_{k=J_{i-1}^{*}+1}^{J_{i}^{*}}\left(\beta_{i} \sqrt{a_{k}}\right)\left(\beta \sqrt{a_{k}}\right)-\sum_{k=1}^{n} x_{k} \beta \sqrt{a_{k}} \\
& =\left\langle\mathfrak{u}_{n}, \mathfrak{m}^{*}\right\rangle-\left\langle x, \mathfrak{m}^{*}\right\rangle \\
& =\phi_{n}\left(\mathfrak{u}_{n}\right)-\phi_{n}(x),
\end{aligned}
$$

segue de (4.2.139) que

$$
\phi_{n}(x) \leq \phi_{n}\left(\mathfrak{u}_{n}\right), \quad \forall x \in \Psi
$$




\subsection{Conclusão}

Nesta seção reuniremos todos os resultados das Seções 4.1 e 4.2 em um argumento para a estimativa do termo $\mathrm{K}_{1}$.

Começamos argumentando que, de (4.1.14), (4.1.24), (4.1.25), (4.1.34) e (4.2.107), para todo $\beta>0$ e todo $\varepsilon>0$, com $\mathbb{P}$-probabilidade $\geq 1-e^{-\varepsilon N}$,

$$
\frac{1}{N} \log \Lambda_{1} \leq F_{n}(\beta)+\beta \sum_{\ell=1}^{\ell_{n}} \beta_{\ell} \sum_{k=J_{\ell-1}^{*}+1}^{J_{\ell}^{*}} a_{k}+\varepsilon,
$$

para todo $N$ grande o suficiente.

De (4.1.1), ainda temos que

$$
\frac{1}{N} \log \mathrm{K}_{1} \leq \frac{1}{N} \log \max _{\ell \in \mathcal{I}_{1}} \Lambda_{\ell}+\frac{\log \mathrm{v}_{1}}{N} .
$$

Seja $\left\{\mathcal{B}_{\varepsilon, N}(\ell)\right\}_{\ell=1}^{v_{1}}$ a sequência de eventos definidos como

$$
\mathcal{B}_{\varepsilon, N}(\ell) \stackrel{\text { def }}{=}\left\{\frac{1}{N} \log \Lambda_{\ell}>F_{n}(\beta)+\beta \sum_{\ell=1}^{\ell_{n}} \beta_{\ell} \sum_{k=J_{\ell-1}^{*}+1}^{J_{\ell}^{*}} a_{k}+\varepsilon\right\} .
$$

Como as variáveis aleatórias $\left\{\Lambda_{\ell}\right\}_{\ell=1}^{v_{1}}$ são identicamente distribuídas, de (4.2.107), para todo $\varepsilon>0$,

$$
\mathbb{P}\left[\bigcup_{\ell \in \mathcal{I}_{\mathrm{v}_{1}}} \mathcal{B}_{\varepsilon, N}^{c}(\ell)\right] \leq \mathrm{v}_{1} \mathbb{P}\left[\mathcal{B}_{\varepsilon, N}^{c}(1)\right] \leq \mathrm{v}_{1} e^{-\varepsilon N} \leq e^{-\frac{\varepsilon}{2} N},
$$

para todo $N$ grande o suficiente. Como o último termo da desigualdade acima é somável, então, pelo Primeiro Lema de Borel-Cantelli, obtemos que

$$
\frac{1}{N} \log \max _{\ell \in \mathcal{I}_{v_{1}}} \Lambda_{\ell} \leq F_{n}(\beta)+\beta \sum_{\ell=1}^{\ell_{n}} \beta_{\ell} \sum_{k=J_{\ell-1}^{*}+1}^{J_{\ell}^{*}} a_{k}+\varepsilon, \quad \mathbb{P}-\text { q.c. }
$$

Juntando (4.3.2) e (4.3.5), para todo $\varepsilon>0$, com $\mathbb{P}$-probabilidade 1,

$$
\frac{1}{N} \log \mathrm{K}_{1} \leq F_{n}(\beta)+\beta \sum_{\ell=1}^{\ell_{n}} \beta_{\ell} \sum_{k=J_{\ell-1}^{*}+1}^{J_{\ell}^{*}} a_{k}+\varepsilon+\frac{\log \mathrm{v}_{1}}{N},
$$

para todo $N$ grande o suficiente.

Agora, tomando primeiro o $\lim \sup \operatorname{com} N \uparrow \infty$, é imediato que

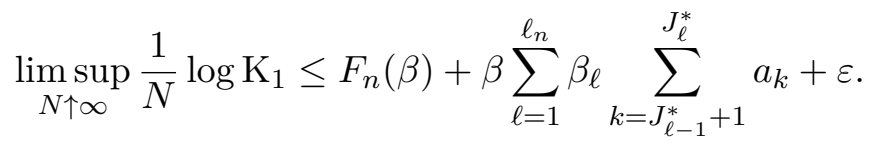

Como a desigualdade acima vale para todo $\varepsilon>0$, concluímos finalmente que, com $\mathbb{P}$-probabilidade 1 ,

$$
\limsup _{N \uparrow \infty} \frac{1}{N} \log \mathrm{K}_{1} \leq F_{n}(\beta)+\beta \sum_{\ell=1}^{\ell_{n}} \beta_{\ell} \sum_{k=J_{\ell-1}^{*}+1}^{J_{\ell}^{*}} a_{k} .
$$




\section{Capítulo 5}

\section{Conclusão da Prova do Teorema 2.3.1}

Este capítulo está dividido em duas seções. Na Seção 5.1 derivaremos uma cota superior para o termo $\mathrm{K}_{2}$, de mesma ordem logarítmica que o termo $\mathrm{K}_{1}$, adaptando o argumento desenvolvido no Capítulo 4. Na Seção 5.2 reuniremos as estimativas do Capítulo 3, 4 e da Seção 5.1 e deduziremos um argumento para concluir a prova do Teorema 2.3.1.

\subsection{Estimativa para $\mathrm{K}_{2}$}

O objeto de estudo desta seção é o termo $\mathrm{K}_{2}$, o qual está definido como

$$
\mathrm{K}_{2}=\max _{e \in \mathcal{G}} \sum_{\gamma_{\boldsymbol{\eta}, \boldsymbol{\eta}^{\prime}} \ni e} e^{-\beta\left[H(\boldsymbol{\eta})+H\left(\boldsymbol{\eta}^{\prime}\right)\right]} \mathbb{1}\left\{\mathrm{d}_{1}\left(\boldsymbol{\eta}, \boldsymbol{\eta}^{\prime}\right)<\kappa \mathrm{v}_{1}\right\}
$$

Nosso objetivo é derivar uma cota para a expressão anterior de mesma ordem logarítmica que o termo $\mathrm{K}_{1}$, cuja estimativa está dada em (4.3.8).

A ideia básica é a seguinte. Voltando à Proposição 3.2.1, para cada par de sítios $\left(\boldsymbol{\eta}, \boldsymbol{\eta}^{\prime}\right)$, tais que $\mathrm{d}_{1}\left(\boldsymbol{\eta}, \boldsymbol{\eta}^{\prime}\right)<\kappa \mathrm{v}_{1}$, o caminho em $\Gamma$ que conecta eles é, com $\mathbb{P}$-probabilidade 1 , da forma

$$
\gamma_{\boldsymbol{\eta}, \boldsymbol{\eta}^{\prime}}=\gamma_{\boldsymbol{\eta}, \boldsymbol{\omega}} \cup \gamma_{\boldsymbol{\omega}, \boldsymbol{\eta}^{\prime}}
$$

em que $\boldsymbol{\omega} \in \Omega_{\mathcal{V}}^{\boldsymbol{\eta}, \boldsymbol{\eta}^{\prime}}$ é o sítio intermediário (cf. Definição 3.2.8) e $\Omega_{\mathcal{V}}^{\boldsymbol{\eta}, \boldsymbol{\eta}^{\prime}}$ está definido em (3.2.36). Agora, como em (5.1.1) a soma está sobre caminhos $\gamma_{\boldsymbol{\eta}, \boldsymbol{\eta}^{\prime}} \in \Gamma$ que passam por meio do elo $e$, então, por causa de (5.1.2) e do fato que os caminhos em $\Gamma$ são auto-evitantes, ou $e \in \gamma_{\boldsymbol{\eta}, \boldsymbol{\omega}}$ ou $e \in \gamma_{\boldsymbol{\omega}, \boldsymbol{\eta}^{\prime}}$. A ideia é proceder a estimativa considerando esses dois casos possíveis, ou seja, quando $e$ aparece antes do sítio intermediário $\boldsymbol{\omega}\left(e \in \gamma_{\boldsymbol{\eta}, \boldsymbol{\omega}}\right)$ e quando $e$ aparece depois do sítio intermediário $\boldsymbol{\omega}\left(e \in \gamma_{\boldsymbol{\omega}, \boldsymbol{\eta}^{\prime}}\right)$.

Começaremos escrevendo

$$
\mathrm{K}_{2} \leq 2\left(\mathrm{~K}_{2}^{\mathrm{a}} \vee \mathrm{K}_{2}^{\mathrm{d}}\right), \quad \mathbb{P}-\text { q.c. }
$$


em que

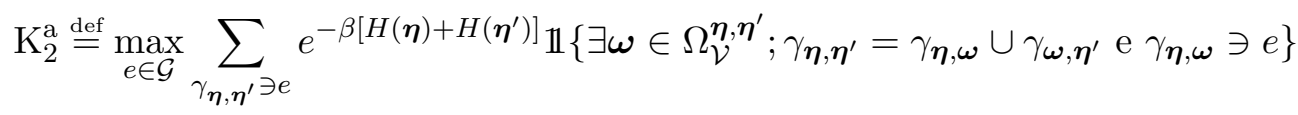

e

$$
\mathrm{K}_{2}^{\mathrm{d}}=\max _{e \in \mathcal{G}} \sum_{\gamma_{\boldsymbol{\eta}, \boldsymbol{\eta}^{\prime}} \ni e} e^{-\beta\left[H(\boldsymbol{\eta})+H\left(\boldsymbol{\eta}^{\prime}\right)\right]} \mathbb{1}\left\{\exists \boldsymbol{\omega} \in \Omega_{\mathcal{V}}^{\boldsymbol{\eta}, \boldsymbol{\eta}^{\prime}} ; \gamma_{\boldsymbol{\eta}, \boldsymbol{\eta}^{\prime}}=\gamma_{\boldsymbol{\eta}, \boldsymbol{\omega}} \cup \gamma_{\boldsymbol{\omega}, \boldsymbol{\eta}^{\prime}} \text { e } \gamma_{\boldsymbol{\omega}, \boldsymbol{\eta}^{\prime}} \ni e\right\}
$$

Seja $e=\left(\boldsymbol{\sigma}, \boldsymbol{\sigma}^{\prime}\right) \in \mathcal{G}$. A primeira estimativa vem do fato que se $e \in \gamma_{\boldsymbol{\omega}, \boldsymbol{\eta}^{\prime}}$, assim a soma em $\mathrm{K}_{2}^{\mathrm{d}}$ está sobre um conjunto de caminhos conectando pares de sítios $\left(\boldsymbol{\eta}, \boldsymbol{\eta}^{\prime}\right)$, tais que $\boldsymbol{\eta}$ está em um hipercubo de dimensão no máximo $V$ em torno de $\boldsymbol{\sigma}$ e $\boldsymbol{\eta}^{\prime}$ está em um hipercubo de dimensão no máximo $2 \kappa v_{1}, \kappa \in\left(0, \frac{1}{2}\right)$, em torno de $\boldsymbol{\sigma}^{\prime}$. Daí, é imediato que

$$
\mathrm{K}_{2}^{\mathrm{d}} \leq 2^{2 \kappa \mathrm{v}_{1}} e^{-\beta H(\overline{\boldsymbol{\sigma}})} Z \stackrel{(2.1 .13)}{\leq} 2^{\mathcal{S}} e^{-\beta H(\overline{\boldsymbol{\sigma}})} Z .
$$

Usando o Teorema 1.5(iii)[BK04a] e o Corolário 2.1.1, com $\mathbb{P}$-probabilidade 1,

$$
\limsup _{N \uparrow \infty} \frac{1}{N} \log \mathrm{K}_{2}^{\mathrm{d}} \leq F_{n}(\beta)+\beta \sum_{\ell=1}^{\ell_{n}} \beta_{\ell} \sum_{k=J_{\ell-1}^{*}+1}^{J_{\ell}^{*}} a_{k} .
$$

Agora, vamos considerar o termo $\mathrm{K}_{2}^{\mathrm{a}}$. Por construção, os caminhos de $\gamma_{\boldsymbol{\eta}, \boldsymbol{\omega}}$ são escolhidos no conjunto $\bigcup_{\ell=1}^{v_{1}} \Gamma^{\ell}$, então,

$$
\begin{aligned}
\mathrm{K}_{2}^{\mathrm{a}} & =\max _{e \in \mathcal{G}} \sum_{\gamma_{\boldsymbol{\eta}, \boldsymbol{\eta}^{\prime}} \ni e} e^{-\beta\left[H(\boldsymbol{\eta})+H\left(\boldsymbol{\eta}^{\prime}\right)\right]} \mathbb{1}\left\{\exists \boldsymbol{\omega} \in \Omega_{\mathcal{V}}^{\boldsymbol{\eta}, \boldsymbol{\eta}^{\prime}} ; \gamma_{\boldsymbol{\eta}, \boldsymbol{\eta}^{\prime}}=\gamma_{\boldsymbol{\eta}, \boldsymbol{\omega}} \cup \gamma_{\boldsymbol{\omega}, \boldsymbol{\eta}^{\prime}} \text { e } \gamma_{\boldsymbol{\eta}, \boldsymbol{\omega}} \ni e\right\} \\
& \leq \mathbf{v}_{1} \max _{\ell \in \mathcal{I}_{\boldsymbol{v}_{1}}} \max _{e \in \mathcal{G}} \sum_{\gamma_{\boldsymbol{\eta}, \boldsymbol{\eta}^{\prime}} \ni e} e^{-\beta\left[H(\boldsymbol{\eta})+H\left(\boldsymbol{\eta}^{\prime}\right)\right]} \mathbb{1}\left\{\exists \boldsymbol{\omega} \in \Omega_{\mathcal{V}}^{\boldsymbol{\eta}, \boldsymbol{\eta}^{\prime}} ; \gamma_{\boldsymbol{\eta}, \boldsymbol{\eta}^{\prime}}=\gamma_{\boldsymbol{\eta}, \boldsymbol{\omega}}^{\ell} \cup \gamma_{\boldsymbol{\omega}, \boldsymbol{\eta}^{\prime}} \text { e } \gamma_{\boldsymbol{\eta}, \boldsymbol{\omega}}^{\ell} \ni e\right\},
\end{aligned}
$$

Vamos denotar por

$$
\Lambda_{\ell}^{\prime} \stackrel{\text { def }}{=} \max _{e \in \mathcal{G}} \sum_{\gamma_{\boldsymbol{\eta}, \boldsymbol{\eta}^{\prime}} \ni e} e^{-\beta\left[H(\boldsymbol{\eta})+H\left(\boldsymbol{\eta}^{\prime}\right)\right]} \mathbb{1}\left\{\exists \boldsymbol{\omega} \in \Omega_{\mathcal{V}}^{\boldsymbol{\eta}, \boldsymbol{\eta}^{\prime}} ; \gamma_{\boldsymbol{\eta}, \boldsymbol{\eta}^{\prime}}=\gamma_{\boldsymbol{\eta}, \boldsymbol{\omega}}^{\ell} \cup \gamma_{\boldsymbol{\omega}, \boldsymbol{\eta}^{\prime}} \text { e } \gamma_{\boldsymbol{\eta}, \boldsymbol{\omega}}^{\ell} \ni e\right\}
$$

para $\ell \in \mathcal{I}_{\mathrm{v}_{1}}$.

Com argumentos análogos ao usados em (4.1.4) para a variável $\Lambda_{\ell}$ (cf. (4.1.2)), podemos concluir que é suficiente considerar apenas a variável $\Lambda_{1}^{\prime}$ aqui. Nesse caso, escrevemos

$$
\begin{aligned}
\Lambda_{1}^{\prime} & =\max _{e \in \mathcal{G}} \sum_{\gamma_{\boldsymbol{\eta}, \boldsymbol{\eta}^{\prime}} \ni e} e^{-\beta\left[H(\boldsymbol{\eta})+H\left(\boldsymbol{\eta}^{\prime}\right)\right]} \mathbb{1}\left\{\exists \boldsymbol{\omega} \in \Omega_{\mathcal{V}}^{\boldsymbol{\eta}, \boldsymbol{\eta}^{\prime}} ; \gamma_{\boldsymbol{\eta}, \boldsymbol{\eta}^{\prime}}=\gamma_{\boldsymbol{\eta}, \boldsymbol{\omega}}^{1} \cup \gamma_{\boldsymbol{\omega}, \boldsymbol{\eta}^{\prime}} \text { e } \gamma_{\boldsymbol{\eta}, \boldsymbol{\omega}}^{1} \ni e\right\} \\
& =\max _{l \in \mathcal{I}_{\mathrm{B}}} \max _{\substack{\boldsymbol{c}\left(\boldsymbol{\sigma}, \boldsymbol{\sigma}^{\prime}\right) \in \mathcal{G} \\
\hat{\imath}(e)=l}} \sum_{\gamma_{\boldsymbol{\eta}, \boldsymbol{\eta}^{\prime}} \ni e} e^{-\beta\left[H(\boldsymbol{\eta})+H\left(\boldsymbol{\eta}^{\prime}\right)\right]} \mathbb{1}\left\{\exists \boldsymbol{\omega} \in \Omega_{\mathcal{V}}^{\boldsymbol{\eta}, \boldsymbol{\eta}^{\prime}} ; \gamma_{\boldsymbol{\eta}, \boldsymbol{\eta}^{\prime}}=\gamma_{\boldsymbol{\eta}, \boldsymbol{\omega}}^{1} \cup \gamma_{\boldsymbol{\omega}, \boldsymbol{\eta}^{\prime}} \text { e } \gamma_{\boldsymbol{\eta}, \boldsymbol{\omega}}^{1} \ni e\right\} .
\end{aligned}
$$


Vamos fixar $e=\left(\boldsymbol{\sigma}, \boldsymbol{\sigma}^{\prime}\right) \in \mathcal{G}$. Seguindo a notação estabelecida na Seção 4.1, a partir da expressão (4.1.9), pela construção dos caminhos em $\Gamma^{1}$, temos que

$$
\left\{(\boldsymbol{\eta}, \boldsymbol{\omega}) \in \Omega_{\mathcal{V}} \times \Omega_{\mathcal{V}}^{\boldsymbol{\eta}, \boldsymbol{\eta}^{\prime}}: \gamma_{\boldsymbol{\eta}, \boldsymbol{\omega}}^{1} \ni e\right\} \subseteq \Omega_{l}^{-}(\boldsymbol{\sigma}) \times \Omega_{l}^{+}\left(\boldsymbol{\sigma}^{\prime}\right)
$$

em que $l=\hat{\imath}(e)$ (cf. Figura 5.1 como auxílio). Estes dois últimos conjuntos podendo ser escritos como em (4.1.17) e (4.1.18).

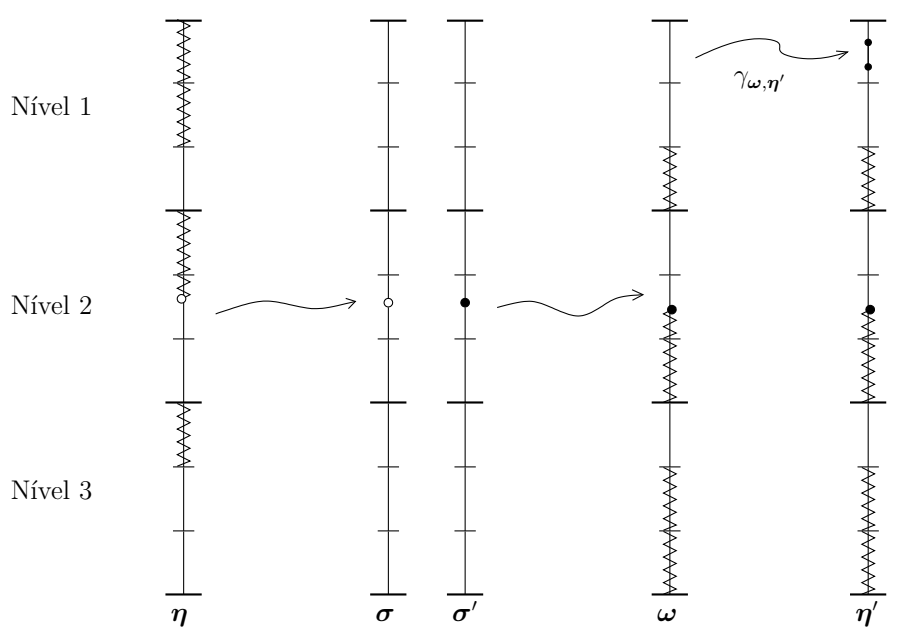

Figura 5.1: A figura acima ilustra um caminho $\gamma_{\boldsymbol{\eta}, \boldsymbol{\eta}^{\prime}}=\gamma_{\boldsymbol{\eta}, \boldsymbol{\omega}}^{1} \cup \gamma_{\boldsymbol{\omega}, \boldsymbol{\eta}^{\prime}} \operatorname{com} \gamma_{\boldsymbol{\eta}, \boldsymbol{\omega}}^{1} \ni e=\left(\boldsymbol{\sigma}, \boldsymbol{\sigma}^{\prime}\right)$. Em $\boldsymbol{\eta}$, respectivamente em $\boldsymbol{\omega}$, a linha em zigue-zague representa os spins possivelmente trocados na construção do caminho $\gamma_{\boldsymbol{\eta}, \boldsymbol{\omega}}^{1}$ antes de $\boldsymbol{\sigma}$, respectivamente depois de $\boldsymbol{\sigma}^{\prime}$. Chamamos atenção ao fato que o caminho $\gamma_{\boldsymbol{\omega}, \boldsymbol{\eta}^{\prime}}$ é obtido trocando-se apenas os spins das coordenadas pertencentes ao conjunto $\mathcal{D}_{\mathcal{V}_{1}(1)}\left(\boldsymbol{\omega}, \boldsymbol{\eta}^{\prime}\right)$ que estão representadas pelas bolas cheias no primeiro bloco de $\boldsymbol{\eta}^{\prime}$.

Agora, por causa de $\boldsymbol{\omega} \in \Omega_{\mathcal{V}}^{\boldsymbol{\eta}, \boldsymbol{\eta}^{\prime}}$ (cf. (3.2.36)), obtemos que

$$
\left.\boldsymbol{\omega}\right|_{\mathcal{V}_{1}^{c}(1)}=\left.\boldsymbol{\eta}^{\prime}\right|_{\mathcal{V}_{1}^{c}(1)} \quad \text { e } \quad \mathrm{d}_{1}\left(\boldsymbol{\omega}, \boldsymbol{\eta}^{\prime}\right)=\mathrm{d}_{\mathrm{H}}\left(\boldsymbol{\omega}, \boldsymbol{\eta}^{\prime}\right) \stackrel{(3.2 .37)}{\leq} 2 \kappa \mathrm{v}_{1}
$$

e como $\kappa \in\left(0, \frac{1}{2}\right)$, o número total de spins discordantes entre $\boldsymbol{\omega}$ e $\boldsymbol{\eta}^{\prime}$ não pode exceder o volume de $\mathcal{V}_{1}(1)$, isto é, $v_{1}$. Estas observações junto com (5.1.11) implicam nas seguintes inclusões:

$$
\begin{aligned}
& \left\{\left(\boldsymbol{\eta}, \boldsymbol{\eta}^{\prime}\right) \in \Omega_{\mathcal{V}}^{2}: \exists \boldsymbol{\omega} \in \Omega_{\mathcal{V}}^{\boldsymbol{\eta}, \boldsymbol{\eta}^{\prime}} ; \gamma_{\boldsymbol{\eta}, \boldsymbol{\eta}^{\prime}}=\gamma_{\boldsymbol{\eta}, \boldsymbol{\omega}}^{1} \cup \gamma_{\boldsymbol{\omega}, \boldsymbol{\eta}^{\prime}} \text { e } \gamma_{\boldsymbol{\eta}, \boldsymbol{\omega}}^{1} \ni e\right\} \\
& \subset \Omega_{l}^{-}(\boldsymbol{\sigma}) \times\left\{\boldsymbol{\eta}^{\prime} \in \Omega_{\mathcal{V}}: \exists \boldsymbol{\omega} \in \Omega_{l}^{+}\left(\boldsymbol{\sigma}^{\prime}\right) \operatorname{com} \mathrm{d}_{1}\left(\boldsymbol{\omega}, \boldsymbol{\eta}^{\prime}\right) \leq 2 \kappa \mathrm{v}_{1} \text { e }\left.\boldsymbol{\eta}^{\prime}\right|_{\mathcal{V}_{1}^{c}(1)}=\left.\boldsymbol{\omega}\right|_{\mathcal{V}_{1}^{c}(1)}\right\} \\
& \subset \Omega_{l}^{-}(\boldsymbol{\sigma}) \times\left\{\boldsymbol{\eta}^{\prime} \in \Omega_{\mathcal{V}}:\left.\boldsymbol{\eta}^{\prime}\right|_{\mathcal{V}_{1}^{c}(1)}=\left.\boldsymbol{\omega}\right|_{\mathcal{V}_{1}^{c}(1)} \text { para algum } \boldsymbol{\omega} \in \Omega_{l}^{+}\left(\boldsymbol{\sigma}^{\prime}\right)\right\} .
\end{aligned}
$$


Portanto,

$$
\begin{aligned}
& \sum_{\gamma_{\boldsymbol{\eta}, \boldsymbol{\eta}^{\prime}} \ni e} e^{-\beta\left[H(\boldsymbol{\eta})+H\left(\boldsymbol{\eta}^{\prime}\right)\right]} \mathbb{1}\left\{\exists \boldsymbol{\omega} \in \Omega_{\mathcal{V}}^{\boldsymbol{\eta}, \boldsymbol{\eta}^{\prime}} ; \gamma_{\boldsymbol{\eta}, \boldsymbol{\eta}^{\prime}}=\gamma_{\boldsymbol{\eta}, \boldsymbol{\omega}}^{1} \cup \gamma_{\boldsymbol{\omega}, \boldsymbol{\eta}^{\prime}} \text { e } \gamma_{\boldsymbol{\eta}, \boldsymbol{\omega}}^{1} \ni e\right\} \\
& \quad \leq \sum_{\boldsymbol{\eta} \in \Omega_{l}^{-}(\boldsymbol{\sigma})} e^{-\beta H(\boldsymbol{\eta})} \sum_{\boldsymbol{\eta}^{\prime} \in \Omega_{\mathcal{V}}} e^{-\beta H\left(\boldsymbol{\eta}^{\prime}\right)} \mathbb{1}\left\{\left.\boldsymbol{\eta}^{\prime}\right|_{\mathcal{V}_{1}^{c}(1)}=\left.\boldsymbol{\omega}\right|_{\mathcal{V}_{1}^{c}(1)} \text { para algum } \boldsymbol{\omega} \in \Omega_{l}^{+}\left(\boldsymbol{\sigma}^{\prime}\right)\right\}
\end{aligned}
$$

Neste ponto, assim como foi no Capítulo 4, consideraremos os casos $l \in\{1$, B $\}$ e $l \in$ $\{2, \ldots, B-1\}$ separadamente. Sendo assim:

(i) se $l=1$ ou $\mathrm{B}$, em (5.1.11), temos que

$$
\left|\Omega_{1}^{-}(\boldsymbol{\sigma})\right| \leq 2^{\mathcal{S}} \quad \text { e } \quad \Omega_{1}^{+}\left(\boldsymbol{\sigma}^{\prime}\right)=\Omega_{\mathcal{V}} \quad \text { ou } \quad \Omega_{\mathrm{B}}^{-}(\boldsymbol{\sigma})=\Omega_{\mathcal{V}} \quad \text { e } \quad\left|\Omega_{\mathrm{B}}^{+}\left(\boldsymbol{\sigma}^{\prime}\right)\right| \leq 2^{\mathcal{S}} .
$$

Daí, para $l=1$,

$$
\begin{aligned}
& \sum_{\boldsymbol{\eta} \in \Omega_{1}^{-}(\boldsymbol{\sigma})} e^{-\beta H(\boldsymbol{\eta})} \sum_{\boldsymbol{\eta}^{\prime} \in \Omega_{\mathcal{V}}} e^{-\beta H\left(\boldsymbol{\eta}^{\prime}\right)} \mathbb{1}\left\{\left.\boldsymbol{\eta}^{\prime}\right|_{\mathcal{V}_{1}^{c}(1)}=\left.\boldsymbol{\omega}\right|_{\mathcal{V}_{1}^{c}(1)} \text { para algum } \boldsymbol{\omega} \in \Omega_{1}^{+}\left(\boldsymbol{\sigma}^{\prime}\right)\right\} \\
& \leq 2^{\mathcal{S}} e^{-\beta H(\overline{\boldsymbol{\sigma}})} \sum_{\boldsymbol{\eta}^{\prime} \in \Omega_{\mathcal{V}}} e^{-\beta H\left(\boldsymbol{\eta}^{\prime}\right)} \underbrace{\mathbb{1}\left\{\left.\boldsymbol{\eta}^{\prime}\right|_{\mathcal{V}_{1}^{c}(1)}=\left.\boldsymbol{\omega}\right|_{\mathcal{V}_{1}^{c}(1)} \text { para algum } \boldsymbol{\omega} \in \Omega_{1}^{+}\left(\boldsymbol{\sigma}^{\prime}\right)\right\}}_{\leq 1} \\
& \quad \leq 2^{\mathcal{S}} e^{-\beta H(\overline{\boldsymbol{\sigma}})} Z ;
\end{aligned}
$$

e para $l=\mathrm{B}$,

$$
\begin{gathered}
\sum_{\boldsymbol{\eta} \in \Omega_{\mathrm{B}}^{-}(\boldsymbol{\sigma})} e^{-\beta H(\boldsymbol{\eta})} \sum_{\boldsymbol{\eta}^{\prime} \in \Omega_{\mathcal{V}}} e^{-\beta H\left(\boldsymbol{\eta}^{\prime}\right)} \mathbb{1}\left\{\left.\boldsymbol{\eta}^{\prime}\right|_{\mathcal{V}_{1}^{c}(1)}=\left.\boldsymbol{\omega}\right|_{\mathcal{V}_{1}^{c}(1)} \text { para algum } \boldsymbol{\omega} \in \Omega_{\mathrm{B}}^{+}\left(\boldsymbol{\sigma}^{\prime}\right)\right\} \\
\leq e^{-\beta H(\overline{\boldsymbol{\sigma}})} Z \underbrace{\sum_{\boldsymbol{\eta}^{\prime} \in \Omega_{\mathcal{V}}} \mathbb{1}\left\{\left.\boldsymbol{\eta}^{\prime}\right|_{\mathcal{V}_{1}^{c}(1)}=\left.\boldsymbol{\omega}\right|_{\mathcal{V}_{1}^{c}(1)} \text { para algum } \boldsymbol{\omega} \in \Omega_{\mathrm{B}}^{+}\left(\boldsymbol{\sigma}^{\prime}\right)\right\}}_{\leq 2^{\mathrm{v}_{1} 2^{\mathcal{S}}}} \\
\leq 2^{\mathrm{v}_{1}} 2^{\mathcal{S}} e^{-\beta H(\overline{\boldsymbol{\sigma}})} Z .
\end{gathered}
$$

Ou seja, para ambos, $l=1$ ou $\mathrm{B}$, obtemos que

$$
\begin{gathered}
\max _{\substack{e=\left(\boldsymbol{\sigma}, \boldsymbol{\sigma}^{\prime}\right) \in \mathcal{G} \\
\hat{\imath}(e)=l}} \sum_{\boldsymbol{\eta} \in \Omega_{l}^{-}(\boldsymbol{\sigma})} e^{-\beta H(\boldsymbol{\eta})} \sum_{\boldsymbol{\eta}^{\prime} \in \Omega_{\mathcal{V}}} e^{-\beta H\left(\boldsymbol{\eta}^{\prime}\right)} \mathbb{1}\left\{\left.\boldsymbol{\eta}^{\prime}\right|_{\mathcal{V}_{1}^{c}(1)}=\left.\boldsymbol{\omega}\right|_{\mathcal{V}_{1}^{c}(1)} \text { para algum } \boldsymbol{\omega} \in \Omega_{l}^{+}\left(\boldsymbol{\sigma}^{\prime}\right)\right\} \\
\leq 2^{\mathrm{v}_{1}} 2^{\mathcal{S}} e^{-\beta H(\overline{\boldsymbol{\sigma}})} Z ;
\end{gathered}
$$

(ii) se $l \in\{2, \ldots, \mathrm{B}-1\}$, os conjuntos em (5.1.11) podem ser escritos (cf. (4.1.26) e (4.1.27)) como

$$
\Omega_{l}^{-}(\boldsymbol{\sigma})=\left(\Omega_{\mathcal{V}_{1}^{1, l-1}} \times \Omega_{\mathcal{V}_{1}^{l, l}} \times\left\{\left.\boldsymbol{\sigma}\right|_{\mathcal{V}_{1}^{l+1, \mathrm{~B}}}\right\}\right) \times \cdots \times\left(\Omega_{\mathcal{V}_{n}^{1, l-1}} \times \Omega_{\mathcal{V}_{n}^{l, l}} \times\left\{\left.\boldsymbol{\sigma}\right|_{\mathcal{V}_{n}^{l+1, \mathrm{~B}}}\right\}\right)
$$


e

$$
\Omega_{l}^{+}\left(\boldsymbol{\sigma}^{\prime}\right)=\left(\left\{\left.\boldsymbol{\sigma}^{\prime}\right|_{\mathcal{V}_{1}^{1, l-1}}\right\} \times \Omega_{\mathcal{V}_{1}^{l, l}} \times \Omega_{\mathcal{V}_{1}^{l+1, \mathrm{~B}}}\right) \times \cdots \times\left(\left\{\left.\boldsymbol{\sigma}^{\prime}\right|_{\mathcal{V}_{n}^{1, l-1}}\right\} \times \Omega_{\mathcal{V}_{n}^{l, l}} \times \Omega_{\mathcal{V}_{n}^{l+1, \mathrm{~B}}}\right) .
$$

Então, usando a aplicação $\varphi_{l}$ indicada em (4.1.28), escrevemos

$$
\begin{aligned}
& \sum_{\boldsymbol{\eta} \in \Omega_{l}^{-}(\boldsymbol{\sigma})} e^{-\beta H(\boldsymbol{\eta})} \sum_{\boldsymbol{\eta}^{\prime} \in \Omega_{\mathcal{V}}} e^{-\beta H\left(\boldsymbol{\eta}^{\prime}\right)} \mathbb{1}\left\{\left.\boldsymbol{\eta}^{\prime}\right|_{\mathcal{V}_{1}^{c}(1)}=\left.\boldsymbol{\omega}\right|_{\mathcal{V}_{1}^{c}(1)} \text { para algum } \boldsymbol{\omega} \in \Omega_{l}^{+}\left(\boldsymbol{\sigma}^{\prime}\right)\right\} \\
\leq & \sum_{\boldsymbol{\eta} \in \Omega_{\mathcal{V}^{1}, l-1}} \sum_{\boldsymbol{\xi} \in \Omega_{\mathcal{V}^{l, l}}} e^{-\beta H\left(\boldsymbol{\varphi}_{l}(\boldsymbol{\eta}, \boldsymbol{\xi}, \boldsymbol{\sigma})\right)} \sum_{\boldsymbol{\xi}^{\prime \prime} \in \Omega_{\mathcal{V}_{1}(1)}} \sum_{\boldsymbol{\xi}^{\prime} \in \Omega_{\mathcal{V}^{l, l}}} \sum_{\boldsymbol{\eta}^{\prime} \in \Omega_{\mathcal{V}^{l+1, B}}} e^{-\beta H\left(\left.\boldsymbol{\xi}^{\prime \prime} \boldsymbol{\varphi}_{l}\left(\boldsymbol{\sigma}^{\prime}, \boldsymbol{\xi}^{\prime}, \boldsymbol{\eta}^{\prime}\right)\right|_{\mathcal{V}_{1}^{c}(1)}\right)} \\
\leq & 2^{2 \mathcal{S} \mathcal{S}} \max _{\boldsymbol{\xi} \in \Omega_{\mathcal{V}^{l, l}}} \sum_{\boldsymbol{\eta} \in \Omega_{\mathcal{V}^{1}, l-1}} e^{-\beta H\left(\boldsymbol{\varphi}_{l}(\boldsymbol{\eta}, \boldsymbol{\xi}, \boldsymbol{\sigma})\right)} \max _{\boldsymbol{\xi}^{\prime} \in \Omega_{\mathcal{V}^{l, l}}} \sum_{\boldsymbol{\xi}^{\prime \prime} \in \Omega_{\mathcal{V}_{1}(1)}} \sum_{\boldsymbol{\eta}^{\prime} \in \Omega_{\mathcal{V}^{l+1, B}}} e^{-\beta H\left(\left.\boldsymbol{\xi}^{\prime \prime} \boldsymbol{\varphi}_{l}\left(\boldsymbol{\sigma}^{\prime}, \boldsymbol{\xi}^{\prime}, \boldsymbol{\eta}^{\prime}\right)\right|_{\mathcal{V}_{1}^{c}(1)}\right)},
\end{aligned}
$$

em que usamos também que $\left|\mathcal{V}^{k, k}\right| \leq \mathcal{S}$ para todo $k \in \mathcal{I}_{\mathrm{B}}$

Juntando a desigualdade anterior e (5.1.21), para todo $l \in\{2, \ldots, \mathrm{B}-1\}$,

$$
\begin{aligned}
& \max _{\substack{e=\left(\boldsymbol{\sigma}, \boldsymbol{\sigma}^{\prime}\right) \in \mathcal{G} \\
\hat{\imath}(e)=l}} \sum_{\boldsymbol{\eta} \in \Omega_{l}^{-}(\boldsymbol{\sigma})} e^{-\beta H(\boldsymbol{\eta})} \sum_{\boldsymbol{\eta}^{\prime} \in \Omega_{\mathcal{V}}} e^{-\beta H\left(\boldsymbol{\eta}^{\prime}\right)} \mathbb{1}\left\{\left.\boldsymbol{\omega}\right|_{\mathcal{V}_{1}^{c}(1)}=\left.\boldsymbol{\eta}^{\prime}\right|_{\mathcal{V}_{1}^{c}(1)} \text { para algum } \boldsymbol{\omega} \in \Omega_{l}^{+}\left(\boldsymbol{\sigma}^{\prime}\right)\right\} \\
& \leq 4^{\mathcal{S}} \max _{\substack{e=\left(\boldsymbol{\sigma}, \boldsymbol{\sigma}^{\prime}\right) \in \mathcal{G} \\
\hat{\imath}(e)=l}} \max _{\boldsymbol{\xi} \in \Omega_{\mathcal{V}^{l, l}}} \sum_{\boldsymbol{\eta} \in \Omega_{\mathcal{V}^{1}, l-1}} e^{-\beta H\left(\boldsymbol{\varphi}_{l}(\boldsymbol{\eta}, \boldsymbol{\xi}, \boldsymbol{\sigma})\right)} \max _{\boldsymbol{\xi}^{\prime} \in \Omega_{\mathcal{V}^{l, l}}} \sum_{\boldsymbol{\xi}^{\prime \prime} \in \Omega_{\mathcal{V}_{1}(1)}} \sum_{\boldsymbol{\eta}^{\prime} \in \Omega_{\mathcal{V}^{l}+1, \mathrm{~B}}} e^{-\beta H\left(\left.\boldsymbol{\xi}^{\prime \prime} \boldsymbol{\varphi}_{l}\left(\boldsymbol{\sigma}^{\prime}, \boldsymbol{\xi}^{\prime}, \boldsymbol{\eta}^{\prime}\right)\right|_{\mathcal{V}_{1}^{c}(1)}\right)} \\
& \leq 2^{\mathrm{v}_{1}} 4^{\mathcal{S}} \max _{\boldsymbol{\xi} \in \Omega_{\mathcal{V}^{l, l}}} \max _{\boldsymbol{\zeta}^{\prime} \in \Omega_{\mathcal{V}}} \sum_{\boldsymbol{\eta} \in \Omega_{\mathcal{V}^{1}, l-1}} e^{-\beta H\left(\boldsymbol{\varphi}_{l}(\boldsymbol{\eta}, \boldsymbol{\xi}, \boldsymbol{\zeta})\right)} \max _{\boldsymbol{\xi}^{\prime} \in \Omega_{\mathcal{V}^{l, l}}} \max _{\boldsymbol{\zeta} \in \Omega_{\mathcal{V}}} \sum_{\boldsymbol{\eta}^{\prime} \in \Omega_{\mathcal{V}^{l}+1, \mathrm{~B}}} e^{-\beta H\left(\boldsymbol{\varphi}_{l}\left(\boldsymbol{\zeta}, \boldsymbol{\xi}^{\prime}, \boldsymbol{\eta}^{\prime}\right)\right)} \\
& =2^{\mathrm{v}_{1}} 4^{\mathcal{S}} \max _{\boldsymbol{\xi}^{\prime} \in \Omega_{\nu l, l}} \max _{\boldsymbol{\zeta}^{\prime} \in \Omega_{\mathcal{\nu}}} Z_{1, l}^{(1)}\left(\boldsymbol{\xi}, \boldsymbol{\zeta}^{\prime}\right) \max _{\boldsymbol{\xi} \in \Omega_{\nu l, l}} \max _{\boldsymbol{\zeta} \in \Omega_{\mathcal{\nu}}} Z_{l, \mathrm{~B}}^{(1)}\left(\boldsymbol{\zeta}, \boldsymbol{\xi}^{\prime}\right)
\end{aligned}
$$

em que usamos a notação indicada em (4.1.32) e (4.1.33) para a igualdade.

Reunindo então (5.1.10), (5.1.14), (5.1.18) e (5.1.22), obtemos que

$$
\Lambda_{1}^{\prime} \leq 2^{\mathrm{v}_{1}}\left(2^{\mathcal{S}} e^{-\beta H(\overline{\boldsymbol{\sigma}})} Z \vee 4^{\mathcal{S}} \max _{l \in\{2, \ldots, \mathrm{B}-1\}} \max _{\boldsymbol{\xi}^{\prime} \in \Omega_{\mathcal{\nu l}, l}} \max _{\boldsymbol{\zeta}^{\prime} \in \Omega_{\mathcal{V}}} Z_{1, l}^{(1)}\left(\boldsymbol{\xi}, \boldsymbol{\zeta}^{\prime}\right) \max _{\boldsymbol{\xi} \in \Omega_{\mathcal{V}^{l, l}}} \max _{\boldsymbol{\zeta} \in \Omega_{\mathcal{V}}} Z_{l, \mathrm{~B}}^{(1)}\left(\boldsymbol{\zeta}, \boldsymbol{\xi}^{\prime}\right)\right) \text {. }
$$

Invocando agora (4.1.25) e (4.2.107), para todo $\beta>0$ e todo $\varepsilon>0$, com $\mathbb{P}$-probabilidade $\geq 1-e^{-\varepsilon N}$

$$
\frac{1}{N} \log \Lambda_{1}^{\prime} \leq F_{n}(\beta)+\beta \sum_{\ell=1}^{\ell_{n}} \beta_{\ell} \sum_{k=J_{\ell-1}^{*}+1}^{J_{\ell}^{*}} a_{k}+\varepsilon,
$$

para todo $N$ grande o suficiente, em que também usamos que $v_{1}$ é $o(N)$. Por fim, usando argumentos similares aos da Seção 4.3, concluímos que, para todo $\beta>0$, com $\mathbb{P}$-probabilidade 1 ,

$$
\limsup _{N \uparrow \infty} \frac{1}{N} \log \mathrm{K}_{2}^{\mathrm{a}} \leq F_{n}(\beta)+\beta \sum_{\ell=1}^{\ell_{n}} \beta_{\ell} \sum_{k=J_{\ell-1}^{*}+1}^{J_{\ell}^{*}} a_{k}
$$


Conclusão da Estimativa de $\mathrm{K}_{2}$. Voltando à (5.1.3), segue de (5.1.7) e (5.1.25) que, para todo $\beta>0$, com $\mathbb{P}$-probabilidade 1 ,

$$
\underset{N \uparrow \infty}{\limsup } \frac{1}{N} \log \mathrm{K}_{2} \leq F_{n}(\beta)+\beta \sum_{\ell=1}^{\ell_{n}} \beta_{\ell} \sum_{k=J_{\ell-1}^{*}+1}^{J_{\ell}^{*}} a_{k} .
$$

\subsection{Conclusão da Prova do Teorema 2.3.1}

Voltando à (3.1.5) e (3.2.46), obtemos que

$$
\tau_{N} \leq \frac{N^{2}}{Z}\left(\mathrm{~K}_{\mathcal{G}} \vee \mathrm{K}_{\mathcal{E} \backslash \mathcal{G}}\right),
$$

em que $\mathrm{K}_{\mathcal{G}}$ e $\mathrm{K}_{\mathcal{E} \backslash \mathcal{G}}$ estão indicados em (3.2.44) e (3.2.45).

Usando agora (3.2.48), (3.2.49) e (3.2.50), com $\mathbb{P}$-probabilidade 1,

$$
\tau_{N} \leq \frac{2 N^{2} e^{\rho \beta V}}{Z}\left(\mathrm{~K}_{1} \vee \mathrm{K}_{2}\right),
$$

para todo $N$ grande o suficiente, em que $\mathrm{K}_{1}$ e $\mathrm{K}_{2}$ estão indicados em (3.2.51) e (3.2.52).

Por fim, juntando (4.3.8), (5.1.26) e o Corolário 2.1.1, obtemos que, para todo $\beta>0$ e todo $\rho>0$, com $\mathbb{P}$-probabilidade 1,

$$
\begin{aligned}
\limsup _{N \uparrow \infty} \frac{1}{N} \log \tau_{N} & \leq F_{n}(\beta)+\beta \sum_{\ell=1}^{\ell_{n}} \beta_{\ell} \sum_{k=J_{\ell-1}^{*}+1}^{J_{\ell}^{*}} a_{k}-F_{n}(\beta)+\rho \beta \\
& =\beta \sum_{\ell=1}^{\ell_{n}} \beta_{\ell} \sum_{k=J_{\ell-1}^{*}+1}^{J_{\ell}^{*}} a_{k}+\rho \beta .
\end{aligned}
$$

Como a desigualdade acima vale para todo $\rho>0$, com $\mathbb{P}$-probabilidade 1 ,

$$
\limsup _{N \uparrow \infty} \frac{1}{N} \log \tau_{N} \leq \beta \sum_{\ell=1}^{\ell_{n}} \beta_{\ell} \sum_{k=J_{\ell-1}^{*}+1}^{J_{\ell}^{*}} a_{k},
$$

isso encerra a demonstração do Teorema 2.3.1.

Como consequência imediata do Teorema 2.3.1, temos o seguinte corolário:

Corolário 5.2.1. Para todo $\beta>0$ e todo $\varepsilon>0$, se

$$
\frac{1}{N} \log \left(t_{N}(\varepsilon, \beta)\right)=2 \varepsilon+\beta \sum_{\ell=1}^{\ell_{n}} \beta_{\ell} \sum_{k=J_{\ell-1}^{*}+1}^{J_{\ell}^{*}} a_{k},
$$

então, com $\mathbb{P}$-probabilidade 1 , para todo $t \geq t_{N}(\varepsilon, \beta)$,

$$
\sup _{\boldsymbol{\sigma}}\left\|\mathrm{P}_{t}(\boldsymbol{\sigma}, \cdot)-\mu(\cdot)\right\|_{\mathrm{var}} \leq \varepsilon,
$$

para todo $N$ grande o suficiente. 
Demonstração. Segue diretamente da combinação do Corolário 2.1.1, Teorema 1.5(iii)[BK04a] e do Teorema 2.3.1. 


\section{Capítulo 6}

\section{Cota Inferior para o Inverso da Lacuna: um caso par- ticular}

\subsection{Introdução e Resultados}

Mesmo que o interesse principal desta tese seja exibir uma cota superior para o inverso da lacuna da dinâmica de Metropolis para o GREM, a pergunta natural que se segue é: o que se sabe sobre uma cota inferior? Esse interesse parece ainda mais verossímil quando levamos em conta o fato que em [FIKP98], onde o caso REM é considerado, tal questão é estudada (cf. Proposição 3.1). Não apenas isso, é mostrado que ambos, cota inferior e superior para $\frac{1}{N} \log \tau_{N}$, convergem $\mathbb{P}$-quase certamente para o mesmo limite quando $N \uparrow \infty$ (cf. Teorema 1$)$.

Neste capítulo continuaremos considerando o GREM definido na Seção 2.1 como um sistema fora do equílibrio sujeito à dinâmica de Metropolis em volume finito. Nosso interesse aqui é

derivar uma cota inferior para $\frac{1}{N} \log \tau_{N}$ e compará-la com a cota superior dada pelo Teorema 2.3.1, a fim de obter alguma informação sobre o seu comportamento assintótico exato. Nossa análise focará apenas um caso particular do modelo, a saber, o GREM com dois níveis sob o regime de baixas temperaturas na fase em cascata (explicaremos abaixo o que esta fase significa).

Formalmente, consideraremos o GREM com $n=2$ níveis configurado com a seguinte escolha de parâmetros. Dados $0<a, p<1$, vamos escolher

$$
\begin{array}{lll}
p_{1}=p & \text { e } & p_{2}=1-p \stackrel{\text { def }}{=} \bar{p} \\
a_{1}=a & \text { e } & a_{2}=1-a \stackrel{\text { def }}{=} \bar{a} .
\end{array}
$$

Também usaremos a seguinte notação: para todo $\boldsymbol{\sigma}=\sigma_{1} \sigma_{2} \in \Omega_{\mathcal{V}}$, denotaremos

$$
H_{1}\left(\sigma_{1}\right) \stackrel{\text { def }}{=} H_{1}(\boldsymbol{\sigma})=-\sqrt{a V} X_{\sigma_{1}}^{1} \quad \text { e } \quad H_{2}(\boldsymbol{\sigma}) \stackrel{\text { def }}{=}-\sqrt{\bar{a} V} X_{\boldsymbol{\sigma}}^{2}
$$


Naturalmente, o Hamiltoniano $H$ é pode ser escrito como

$$
H(\boldsymbol{\sigma})=H_{1}\left(\sigma_{1}\right)+H_{2}(\boldsymbol{\sigma}) .
$$

Uma descrição do que é a fase em cascata no GREM segue. Primeiro, ordenamos as configurações de $\Omega_{\mathcal{V}}$ em cascata de acordo com a magnitude das energias $H_{1}$ e $H_{2}$ da seguinte maneira: $\boldsymbol{\sigma}^{1}, \boldsymbol{\sigma}^{2}, \ldots, \boldsymbol{\sigma}^{2^{V}}$ são ordenados considerando que

$$
H_{1}\left(\sigma_{1}^{1}\right)<H_{1}\left(\sigma_{1}^{2}\right)<\cdots<H_{1}\left(\sigma_{1}^{2^{V_{1}}}\right)
$$

e, para cada $j=1, \ldots, 2^{V_{1}}$,

$$
H_{2}\left(\sigma_{1}^{j} \sigma_{2}^{j, 1}\right)<H_{2}\left(\sigma_{1}^{j} \sigma_{2}^{j, 2}\right)<\cdots<H_{2}\left(\sigma_{1}^{j} \sigma_{2}^{j, 2^{V_{2}}}\right) .
$$

A fase em cascata no GREM significa que, para algum $k$ finito, $\boldsymbol{\sigma}^{1}, \boldsymbol{\sigma}^{2}, \ldots, \boldsymbol{\sigma}^{k}$ compõem o conjunto de configurações de energia mínima do sistema, mais precisamente, em temperaturas baixas, a medida de Gibbs concentra-se em $\boldsymbol{\sigma}^{1}, \boldsymbol{\sigma}^{2}, \ldots, \boldsymbol{\sigma}^{k}$ (cf. Teorema 1.9 em [BK04a]).

De modo geral, a fase em cascata em um GREM com 2 níveis é caracterizado pela desigualdade

$$
p_{1} a_{2}<a_{1} p_{2} .
$$

Com a escolha (6.1.1), a condição (6.1.6) reduz-se apenas a:

$$
p<a .
$$

É importante também mencionar que, sob a fase em cascata, $\ell_{2}=2$, isto é, existem duas temperaturas inversas críticas, $\beta_{1}$ e $\beta_{2}\left(\operatorname{com} \beta_{1}<\beta_{2}\right)$, dadas por

$$
\beta_{1}^{2}=\beta_{\star}^{2} \frac{p}{a} \quad \text { e } \quad \beta_{2}^{2}=\beta_{\star}^{2} \frac{\bar{p}}{\bar{a}}, \quad \beta_{\star}=\sqrt{2 \log 2 .}
$$

Portanto, em todo este capítulo, estaremos assumindo que

$$
p<a \quad \text { e } \beta \geq \beta_{2} .
$$

O primeiro resultado deste capítulo é o seguinte teorema.

Teorema 6.1.1. Para todo $\beta>0$, com $\mathbb{P}$-probabilidade 1,

$$
\liminf _{N \uparrow \infty} \frac{1}{\beta N} \log \tau_{N} \geq \beta_{2} \bar{a} .
$$

Demonstração. Seção 6.2. 
Observação 6.1.1. Uma motivação, heurística, para o resultado do Teorema 6.1.1 segue. Partimos do fato que $\tau_{N}$ deveria ser maior que o tempo que o sistema leva para sair de sua configuração inicial. Em particular, da configuração de mínima energia $\overline{\boldsymbol{\sigma}}=\bar{\sigma}_{1} \bar{\sigma}_{2}$. Sob a dinâmica de Metropolis, isso deveria ser da ordem de $\exp \left\{-\beta\left(H\left(\overline{\boldsymbol{\sigma}}^{\prime \prime}\right)-H(\overline{\boldsymbol{\sigma}})\right)\right\}$, em que $\overline{\boldsymbol{\sigma}}^{\prime \prime}=\bar{\sigma}_{1}^{\prime \prime} \bar{\sigma}_{2}^{\prime \prime}$ denota a configuração de mínima energia entre os $V$ vizinhos de $\overline{\boldsymbol{\sigma}}$. Como veremos abaixo, com alta probabilidade, $\overline{\boldsymbol{\sigma}}^{\prime \prime}$ é tal que $\bar{\sigma}_{1}^{\prime \prime}=\bar{\sigma}_{1}$. Daí $H\left(\overline{\boldsymbol{\sigma}}^{\prime \prime}\right)-H(\overline{\boldsymbol{\sigma}})=H_{2}\left(\overline{\boldsymbol{\sigma}}^{\prime \prime}\right)-H_{2}(\overline{\boldsymbol{\sigma}})$, implicando o lado direito de (6.1.10).

Observe agora que o Teorema 2.3.1, sob o contexto que estamos considerando para o GREM, nos dá o seguinte corolário:

Corolário 6.1.1. Para todo $\beta>0$, com $\mathbb{P}$-probabilidade 1 ,

$$
\limsup _{N \uparrow \infty} \frac{1}{\beta N} \log \tau_{N} \leq \beta_{1} a+\beta_{2} \bar{a} .
$$

Combinando então as cotas obtidas no Teorema 6.1.1 e Corolário 6.1.1, obtemos o seguinte corolário:

Corolário 6.1.2. Para todo $\beta>0$, com $\mathbb{P}$-probabilidade 1 ,

$$
\beta_{2} \bar{a} \leq \liminf _{N \uparrow \infty} \frac{1}{\beta N} \log \tau_{N} \leq \limsup _{N \uparrow \infty} \frac{1}{\beta N} \log \tau_{N} \leq \beta_{1} a+\beta_{2} \bar{a} .
$$

Observe que o resultado anterior não implica que o $\operatorname{limite} \lim _{N \uparrow \infty} \frac{1}{N} \log \tau_{N}$ não exista $\mathbb{P}$ quase certamente, mas é um indicativo de que podemos ter subestimado a cota inferior ou superestimado o termo $\mathrm{K}=\mathrm{K}_{N}(\Gamma)$ dado em (3.1.7).

Na verdade, há motivo para crer que a cota correta não seja aquela dada em (6.1.11). A motivação para essa crença segue de [FG18], em que se estuda este mesmo GREM (2 níveis, fase em cascata e temperaturas baixas) sob uma dinâmica de Glauber, que é uma versão simplificada da dinâmica de Metropolis. Entende-se em [FG18] o limite de escala da dinâmica em uma escala de tempo tal que o limite é uma dinâmica ergódica, e logo, deveria estar na mesma escala que o inverso da lacuna espectral. Para todo $\beta \geq \beta_{2}$, temos uma escala de tempo mais curta em [FG18] do que aquela correspondente ao lado direito de (6.1.11). Em certas situações, ocorre que esta escala de tempo se comporta como a do lado direito de (6.1.10); em outras situações, ela é maior, mas sempre menor do que a do lado direito de (6.1.11). Dessa forma, não vemos motivo para esperar um comportamento diferente na escala da lacuna espectral entre as duas dinâmicas.

Por outro lado, também há motivo para crer que a cota superior fornecida pelo Corolário 6.1.1 não pode ser melhorada. Isso segue do Teorema 6.1.2 a seguir, onde provamos que, sob a 
condição de que os caminhos não tenham comprimento maior do que $c N$, para alguma constante positiva $c$, se $\beta \geq \beta_{2}$, então a cota no lado direito de (6.1.11) não pode ser menor.

Teorema 6.1.2. Seja $\Upsilon_{c N}$ a classe de todos os conjuntos completos de caminhos cujos elementos tem comprimento no máximo $c N$, para alguma constante positiva. Então, para todo $\Gamma \in \Upsilon_{c N} \mathrm{e}$ todo $\beta \geq \beta_{2}$, com $\mathbb{P}$-probabilidade 1 ,

$$
\liminf _{N \uparrow \infty} \frac{1}{\beta N} \log K_{N}(\Gamma) \geq \beta_{1} a+\beta_{2} \bar{a} .
$$

Demonstração. Seção 6.3.

\subsection{Demonstração do Teorema 6.1.1}

Começaremos relembrando que a caracterização minimax de autovalores, brevemente descrito na Seção 3.1, nos dá que o inverso da lacuna $\tau_{N}$ é dado por

$$
\tau_{N}=\sup \left\{\tau_{N}(\phi): \phi \text { não constante }\right\}
$$

em que

$$
\tau_{N}(\phi)=\frac{\operatorname{Var}_{\mu}(\phi)}{\mathscr{E}(\phi, \phi)}
$$

sendo

$$
\operatorname{Var}_{\mu}(\phi)=\frac{1}{2} \sum_{\boldsymbol{\sigma}, \boldsymbol{\sigma}^{\prime}}\left(\phi(\boldsymbol{\sigma})-\phi\left(\boldsymbol{\sigma}^{\prime}\right)\right)^{2} \mu(\boldsymbol{\sigma}) \mu\left(\boldsymbol{\sigma}^{\prime}\right)
$$

e

$$
\mathscr{E}(\phi, \phi)=\frac{1}{2} \sum_{\boldsymbol{\sigma}, \boldsymbol{\sigma}^{\prime}}\left(\phi(\boldsymbol{\sigma})-\phi\left(\boldsymbol{\sigma}^{\prime}\right)\right)^{2} \mu(\boldsymbol{\sigma}) \mathrm{P}\left(\boldsymbol{\sigma}, \boldsymbol{\sigma}^{\prime}\right)
$$

A estratégia que usaremos para provar o Teorema 6.1.1 é essencialmente a mesma que foi utilizada em [FIKP98] (cf. Seção 3) em análise similar para o REM e consiste em escolher uma função real não constante $\phi$ sobre $\Omega_{\mathcal{V}}$ e com ela obter um limitante inferior para o lado direito de $(6.2 .2)$.

Como em [FIKP98], escolhemos para $\phi$ o indicador da (única) configuração de mínima energia do sistema, isto é, consideraremos a função

$$
\phi_{\bar{\sigma}}(\boldsymbol{\sigma}) \stackrel{\text { def }}{=} \mathbb{1}\{\boldsymbol{\sigma}=\overline{\boldsymbol{\sigma}}\}
$$

Com a função $\phi_{\overline{\boldsymbol{\sigma}}}$, sob a dinâmica de Metropolis, (2.3.2) fica explicitamente escrito como

$$
\tau_{N}\left(\phi_{\overline{\boldsymbol{\sigma}}}\right)=\frac{V e^{-\beta H(\overline{\boldsymbol{\sigma}})}(1-\mu(\overline{\boldsymbol{\sigma}}))}{\sum_{\boldsymbol{\sigma} \sim \overline{\boldsymbol{\sigma}}} e^{-\beta H(\boldsymbol{\sigma})}},
$$


em que a soma está sobre os $V$ vizinhos próximos de $\overline{\boldsymbol{\sigma}}$ que denotamos por $\boldsymbol{\sigma}$. Aqui, $\boldsymbol{\sigma} \sim \boldsymbol{\sigma}^{\prime}$ significa que $\mathrm{d}_{\mathrm{H}}\left(\boldsymbol{\sigma}, \boldsymbol{\sigma}^{\prime}\right)=1$. Logo, de (2.3.1), não é difícil concluir que

$$
\tau_{N} \geq \tau_{N}\left(\phi_{\overline{\boldsymbol{\sigma}}}\right) \geq \frac{e^{-\beta H_{2}(\overline{\boldsymbol{\sigma}})}(1-\mu(\overline{\boldsymbol{\sigma}}))}{e^{\beta H_{1}\left(\bar{\sigma}_{1}\right)} \sum_{\boldsymbol{\sigma} \sim \overline{\boldsymbol{\sigma}}} e^{-\beta\left[H_{1}\left(\sigma_{1}\right)+H_{2}(\boldsymbol{\sigma})\right]}},
$$

e portanto,

$$
\begin{aligned}
\liminf _{N \uparrow \infty} \frac{1}{N} \log \tau_{N} \geq & \liminf _{N \uparrow \infty} \frac{1}{N} \log e^{-\beta H_{2}(\overline{\boldsymbol{\sigma}})}+\liminf _{N \uparrow \infty} \frac{1}{N} \log (1-\mu(\overline{\boldsymbol{\sigma}})) \\
& +\liminf _{N \uparrow \infty} \frac{1}{N} \log \left(e^{\beta H_{1}\left(\bar{\sigma}_{1}\right)} \sum_{\boldsymbol{\sigma} \sim \overline{\boldsymbol{\sigma}}} e^{-\beta\left[H_{1}\left(\sigma_{1}\right)+H_{2}(\boldsymbol{\sigma})\right]}\right)^{-1} .
\end{aligned}
$$

Para lidar com as duas primeiras parcelas do lado direito da desigualdade anterior usaremos o Lema 6.2.1, a seguir, cuja prova segue de uma adaptação de argumentos usados na demonstração da Proposição 3.1 de [FIKP98].

Lema 6.2.1. Para todo $\beta>0$, com $\mathbb{P}$-probabilidade 1 ,

$$
\liminf _{N \uparrow \infty} \frac{1}{N} \log e^{-\beta H_{2}(\overline{\boldsymbol{\sigma}})} \geq \beta \beta_{2} \bar{a} .
$$

e

$$
\liminf _{N \uparrow \infty} \frac{1}{N} \log (1-\mu(\overline{\boldsymbol{\sigma}}))=0
$$

Agora considere o denominador do lado direito de (6.2.7). Vamos escrever

$$
\begin{aligned}
& e^{\beta H_{1}\left(\bar{\sigma}_{1}\right)} \sum_{\boldsymbol{\sigma} \sim \overline{\boldsymbol{\sigma}}} e^{-\beta\left[H_{1}\left(\sigma_{1}\right)+H_{2}(\boldsymbol{\sigma})\right]} \\
& =e^{\beta H_{1}\left(\bar{\sigma}_{1}\right)}\left(\sum_{\substack{\boldsymbol{\sigma} \sim \overline{\boldsymbol{\sigma}} \\
\sigma_{1} \neq \bar{\sigma}_{1}}} e^{-\beta\left[H_{1}\left(\sigma_{1}\right)+H_{2}(\boldsymbol{\sigma})\right]}+\sum_{\substack{\boldsymbol{\sigma} \sim \overline{\boldsymbol{\sigma}} \\
\sigma 2 \neq \bar{\sigma}_{2}}} e^{-\beta\left[H_{1}\left(\sigma_{1}\right)+H_{2}(\boldsymbol{\sigma})\right]}\right) .
\end{aligned}
$$

Como $\overline{\boldsymbol{\sigma}}=\bar{\sigma}_{1} \bar{\sigma}_{2}$, se $\boldsymbol{\sigma} \sim \overline{\boldsymbol{\sigma}}$ e $\sigma_{2} \neq \bar{\sigma}_{2}$, então $\bar{\sigma}_{1}=\sigma_{1}$ e

$$
e^{\beta H_{1}\left(\bar{\sigma}_{1}\right)} \sum_{\substack{\boldsymbol{\sigma} \sim \overline{\boldsymbol{\sigma}} \\ \sigma_{2} \neq \bar{\sigma}_{2}}} e^{-\beta\left[H_{1}\left(\sigma_{1}\right)+H_{2}(\boldsymbol{\sigma})\right]}=\sum_{\sigma_{2} \sim \bar{\sigma}_{2}} e^{-\beta H_{2}\left(\bar{\sigma}_{1} \sigma_{2}\right)} .
$$

Por outro lado, se $\boldsymbol{\sigma} \sim \overline{\boldsymbol{\sigma}}$ e $\sigma_{1} \neq \bar{\sigma}_{1}$, como $\bar{\sigma}_{1}$ é a configuração em $\Omega_{\mathcal{V}_{1}}$ que minimiza $H_{1}(\cdot)$, temos que

$$
\begin{aligned}
e^{\beta H_{1}\left(\bar{\sigma}_{1}\right)} \sum_{\substack{\boldsymbol{\sigma} \sim \overline{\bar{\sigma}} \\
\sigma 1 \neq \bar{\sigma}_{1}}} e^{-\beta\left[H_{1}\left(\sigma_{1}\right)+H_{2}(\boldsymbol{\sigma})\right]} & =\sum_{\substack{\boldsymbol{\sigma} \sim \overline{\bar{\sigma}} \\
\sigma_{1} \neq \bar{\sigma}_{1}}} \underbrace{\beta\left[H_{1}\left(\bar{\sigma}_{1}\right)-H_{1}\left(\sigma_{1}\right)\right]}_{\leq 1} e^{-\beta H_{2}\left(\sigma_{1} \bar{\sigma}_{2}\right)} \\
& \leq \sum_{\sigma_{1} \sim \bar{\sigma}_{1}} e^{-\beta H_{2}\left(\sigma_{1} \bar{\sigma}_{2}\right)} .
\end{aligned}
$$


Então, de (6.2.12) e (6.2.13),

$$
e^{\beta H_{1}\left(\bar{\sigma}_{1}\right)} \sum_{\boldsymbol{\sigma} \sim \overline{\boldsymbol{\sigma}}} e^{-\beta\left[H_{1}\left(\sigma_{1}\right)+H_{2}(\boldsymbol{\sigma})\right]} \leq \sum_{\sigma_{1} \sim \bar{\sigma}_{1}} e^{-\beta H_{2}\left(\sigma_{1} \bar{\sigma}_{2}\right)}+\sum_{\sigma_{2} \sim \bar{\sigma}_{2}} e^{-\beta H_{2}\left(\bar{\sigma}_{1} \sigma_{2}\right)}
$$

Neste ponto, usamos a mesma ideia que [FIKP98] e estimamos por cima a primeira soma (respectivamente a segunda) em (6.2.14) por $V_{1}$ (respectivamente por $V_{2}$ ) vezes o máximo dos somandos. Como $\bar{\sigma}_{1}$ (respectivamente $\bar{\sigma}_{2}$ ) é a configuração que minimiza $H_{1}(\cdot)$ (respectivamente $\left.H_{2}\left(\bar{\sigma}_{1} \cdot\right)\right)$, esses somandos não são independentes. Mas o máximo é estocasticamente dominado pelo máximo de $V_{1}$ (respectivamente de $V_{2}$ ) somandos independentes. Este fato segue do Lema B.0.3. Portanto,

$$
\sum_{\sigma_{1} \sim \bar{\sigma}_{1}} e^{-\beta H_{2}\left(\sigma_{1} \bar{\sigma}_{2}\right)}+\sum_{\sigma_{2} \sim \bar{\sigma}_{2}} e^{-\beta H_{2}\left(\bar{\sigma}_{1} \sigma_{2}\right)} \leq V_{1} e^{-\beta m_{1}}+V_{2} e^{-\beta m_{2}},
$$

para todo $N$ grande o suficiente, em que $m_{j}$ denota o mínimo de $V_{j}$ variáveis aleatórias i.i.d. gaussianas padrão. Aqui, usamos também que $a V, \bar{a} V \geq 1$ para $N$ grande.

Pelo Lema B.0.4, para todo $\varepsilon>0$, com $\mathbb{P}$-probabilidade 1,

$$
m_{j} \geq-\sqrt{2(1+\varepsilon) \log V_{j}},
$$

para todo $N$ grande o suficiente. Logo, é imediato que, com $\mathbb{P}$-probabilidade 1 ,

$$
V_{1} e^{-\beta m_{1}}+V_{2} e^{-\beta m_{2}} \leq V e^{-\beta m_{1} \wedge m_{2}} \leq N e^{\beta \sqrt{2(1+\varepsilon) \log N}},
$$

para todo $N$ grande o suficiente.

Usando agora o fato clássico que se duas famílias $\left\{X_{k}\right\}_{k \in \mathbb{N}}$ e $\left\{Y_{k}\right\}_{k \in \mathbb{N}}$ de variáveis aleatórias reais são tais que para todo $k \in \mathbb{N}, X_{k}$ é estocasticamente dominada por $Y_{k}$, então podemos construir um espaço de probabilidade, tal que, $\mathbb{P}$-quase certamente, $X_{k} \leq Y_{k}$ para todo $k \in \mathbb{N}$, de $(6.2 .14),(6.2 .15),(6.2 .16)$ e (6.2.17), com $\mathbb{P}$-probabilidade 1 ,

$$
\liminf _{N \uparrow \infty} \frac{1}{N} \log \left(e^{\beta H_{1}\left(\bar{\sigma}_{1}\right)} \sum_{\boldsymbol{\sigma} \sim \overline{\boldsymbol{\sigma}}} e^{-\beta\left[H_{1}\left(\sigma_{1}\right)+H_{2}(\boldsymbol{\sigma})\right]}\right)^{-1} \geq 0 .
$$

Portanto, do Lema 6.2.1 e (6.2.18), para todo $\beta>0$, com $\mathbb{P}$-probabilidade 1 ,

$$
\liminf _{N \uparrow \infty} \frac{1}{N} \log \tau_{N} \geq \beta \beta_{2} \bar{a} .
$$

\subsection{Demonstração do Teorema 6.1.2}

Antes de começarmos a demonstração, relembremos que, para a dinâmica de Metropolis, $\mathrm{K}_{N}(\Gamma)$ é dado por

$$
\mathrm{K}_{N}(\Gamma)=\frac{V}{Z} \max _{e=\left(\boldsymbol{\sigma}, \boldsymbol{\sigma}^{\prime}\right)} e^{\beta\left[H(\boldsymbol{\sigma}) \vee H\left(\boldsymbol{\sigma}^{\prime}\right)\right]} \sum_{\gamma_{\boldsymbol{\eta}, \boldsymbol{\eta}^{\prime}} \ni e}\left|\gamma_{\boldsymbol{\eta}, \boldsymbol{\eta}^{\prime}}\right| e^{-\beta\left[H(\boldsymbol{\eta})+H\left(\boldsymbol{\eta}^{\prime}\right)\right]},
$$


em que o somatório está sobre todos os caminhos $\gamma_{\boldsymbol{\eta}, \boldsymbol{\eta}^{\prime}}$ em $\Gamma$, exatamente um para cada par de vértices distintos $\boldsymbol{\eta}, \boldsymbol{\eta}^{\prime} \in \Omega_{\mathcal{V}}$.

O termo $\mathrm{K}_{N}(\Gamma)$ depende da escolha do conjunto completo de caminhos $\Gamma$. Vamos restringir a escolha de $\Gamma$ à $\Upsilon_{c N}$ à classe de todos os conjuntos completos de caminhos cujos elementos tem comprimento no máximo $c N$, para alguma constante positiva $c$.

Uma breve descrição da estratégia da demonstração do teorema segue. Partiremos do fato que, por causa do regime de parâmetros que estamos assumindo para o GREM, com $\mathbb{P}$ probabilidade $1, H(\overline{\boldsymbol{\sigma}})$ e $H\left(\overline{\boldsymbol{\sigma}}^{\prime}\right)$, em que $\overline{\boldsymbol{\sigma}}^{\prime}=\bar{\sigma}_{1}^{\prime} \bar{\sigma}_{2}^{\prime}$ é configuração de spins, tal que $\bar{\sigma}_{1}^{\prime}$ é o segundo mínimo de $H_{1}(\cdot)$ e $\bar{\sigma}_{2}^{\prime}$ é o mínimo de $H_{2}\left(\bar{\sigma}_{1}^{\prime} \cdot\right)$, são da mesma ordem logarítmica que a energia livre do sistema. Como $\Gamma$ é um conjunto completo de caminhos, não há como evitar que o caminho $\gamma_{\overline{\boldsymbol{\sigma}}, \overline{\boldsymbol{\sigma}}^{\prime}}$ pertença a $\Gamma$. Isso implica que

$$
\sum_{\gamma_{\boldsymbol{\eta}, \boldsymbol{\eta}^{\prime}} \ni e}\left|\gamma_{\boldsymbol{\eta}, \boldsymbol{\eta}^{\prime}}\right| e^{-\beta\left[H(\boldsymbol{\eta})+H\left(\boldsymbol{\eta}^{\prime}\right)\right]} \geq e^{-\beta\left[H(\overline{\boldsymbol{\sigma}})+H\left(\overline{\boldsymbol{\sigma}}^{\prime}\right)\right]}
$$

para qualquer escolha de $\Gamma$. Por fim, garantiremos que o termo $e^{\beta\left[H(\boldsymbol{\sigma}) \vee H\left(\boldsymbol{\sigma}^{\prime}\right)\right]}$ não é pequeno, em outras palavras, provaremos que no interior do caminho $\gamma_{\overline{\boldsymbol{\sigma}}, \overline{\boldsymbol{\sigma}}^{\prime}}$, com $\mathbb{P}$-probabilidade 1 , existe um sítio com energia no mínimo $-\delta N$, qualquer que seja $\delta>0$ dado. (6.1.13) então segue.

Começaremos definindo, para $x \in \mathbb{R}$, a função

$$
u_{j}(x) \stackrel{\text { def }}{=} \beta_{\star} \sqrt{V_{j}}+\frac{x}{\beta_{\star} \sqrt{V_{j}}}-\frac{1}{2} \frac{\log \left(V_{j} \log 2\right)+\log 4 \pi}{\beta_{\star} \sqrt{V_{j}}}, \quad j=1,2,
$$

e os conjuntos

$$
\mathcal{T}_{1} \stackrel{\text { def }}{=} \mathcal{T}_{1}(x)=\left\{\sigma_{1} \in \Omega_{1}: X_{\sigma_{1}}^{1} \geq u_{1}(x)\right\}
$$

$\mathrm{e}$

$$
\mathcal{T}_{2} \stackrel{\text { def }}{=} \mathcal{T}_{2}(x)=\left\{\sigma_{2} \in \Omega_{2}: X_{\eta_{1} \sigma_{2}}^{2} \geq u_{2}(x), \eta_{1} \in \mathcal{T}_{1}\right\}
$$

Por fim, seja $\mathcal{T}$ o conjunto definido como

$$
\mathcal{T} \stackrel{\text { def }}{=} \mathcal{T}(x)=\mathcal{T}_{1} \times \mathcal{T}_{2}
$$

Observação 6.3.1. É bem conhecido que com $\mathbb{P}$-probabilidade 1 , como segue do regime de parâmetros que estamos assumindo para o GREM, $\mathcal{T} \neq \varnothing$. De fato, veja Teorema 1.5 em [BK04a] por exemplo,

$$
\lim _{x \rightarrow-\infty} \liminf _{N \uparrow \infty} \mathbb{P}\left[\left\{\boldsymbol{\sigma}, \boldsymbol{\sigma}^{\prime}\right\} \subset \mathcal{T}\right]=1
$$

O primeiro passo da nossa demonstração é o seguinte lema. 
Lema 6.3.1. Com $\mathbb{P}$-probabilidade 1 , para todo $0<\varepsilon<\frac{1}{2}$ e para todo $\eta_{1}, \eta_{1}^{\prime} \in \mathcal{T}_{1}$ distintos,

$$
\mathrm{d}_{\mathrm{H}}\left(\eta_{1}, \eta_{1}^{\prime}\right)>\varepsilon V_{1},
$$

para todo $N$ suficientemente grande.

Demonstração. Segue da combinação dos Lema 2.11 e 2.12 em [Gay92].

Para a sequência da demonstração, precisaremos de algumas definições adicionais. Dizemos que um sítio $\boldsymbol{\sigma} \in \Omega_{\mathcal{V}}$ é inútil se, para todo $\delta>0$, existe $j=1,2$ tal que $\left|H_{j}\left(\sigma_{1} \sigma_{j}\right)\right|>\delta N$. Caso contrário, diremos que ele é útil. Diremos que um caminho $\gamma$ é inútil se todos os seus pontos interiores $\gamma^{\text {int }}$ forem inúteis.

O resultado a seguir mostra que no interior de qualquer caminho $\gamma_{\boldsymbol{\eta}, \boldsymbol{\eta}^{\prime}} \in \Gamma$ entre duas configurações $\boldsymbol{\eta}, \boldsymbol{\eta}^{\prime} \in \mathcal{T}$, tais que $\eta_{1} \neq \eta_{1}^{\prime}$, existe, com uma altíssima probabilidade, ao menos um sítio útil, para todo $N$ grande o suficiente.

Lema 6.3.2. Sejam $\boldsymbol{\eta}, \boldsymbol{\eta}^{\prime} \in \mathcal{T}$, tais que $\eta_{1} \neq \eta_{1}^{\prime}$ fixados. Então, com $\mathbb{P}$-probabilidade 1, para todo caminho $\gamma_{\boldsymbol{\eta}, \boldsymbol{\eta}^{\prime}}$ de comprimento no máximo $c N$, para alguma constante $c>0$, para todo $N$ grande o suficiente, existe ao menos um sítio em $\gamma_{\boldsymbol{\eta}, \boldsymbol{\eta}^{\prime}}^{\text {int }}$ que é útil.

Demonstração. Como $\eta_{1} \neq \eta_{1}^{\prime}$, pelo Lema 6.3.1, para todo $0<\varepsilon<\frac{1}{2}$,

$$
\mathrm{d}_{1}\left(\eta_{1}, \eta_{1}^{\prime}\right)>\varepsilon V_{1},
$$

para todo $N$ grande o suficiente. Logo, para todo $N$ grande, existem ao menos $\varepsilon V_{1}$ sítios no interior de $\gamma_{\boldsymbol{\eta}, \boldsymbol{\eta}^{\prime}}$ cujos Hamiltonianos são variáveis aleatórias independentes.

Portanto, fixados $\boldsymbol{\eta}, \boldsymbol{\eta}^{\prime}$ e o caminho $\gamma_{\boldsymbol{\eta}, \boldsymbol{\eta}^{\prime}}$ de $\boldsymbol{\eta}$ a $\boldsymbol{\eta}^{\prime}$ de comprimento maior ou igual do que $\varepsilon V_{1}$, usando a desigualdade em (3.2.21), obtemos que

$$
\begin{aligned}
\mathbb{P}\left[\gamma_{\boldsymbol{\eta}, \boldsymbol{\eta}^{\prime}} \text { ser inútil }\right] & =\mathbb{P}\left[\bigcap_{\boldsymbol{\sigma} \in \gamma_{\boldsymbol{\eta}, \boldsymbol{\eta}^{\prime}}^{\text {int }}}\{\boldsymbol{\sigma} \text { ser inútil }\}\right] \\
& \leq\left(\mathbb{P}\left[\exists j=1,2:\left|H_{j}\left(\sigma_{1} \sigma_{j}\right)\right|>\delta N\right]\right)^{\varepsilon V_{1}} \\
& \leq e^{-c^{\prime} V_{1}^{2}}
\end{aligned}
$$

para todo $N$ grande o suficiente e alguma constante $c^{\prime}>0$ que depende de $\delta$ e $\varepsilon$.

Como para o par de vértices $\boldsymbol{\eta}$ e $\boldsymbol{\eta}^{\prime}$ fixados existe exatamente um caminho em cada $\Gamma \in \Upsilon_{c N}$ conectando-os, de (6.3.10), obtemos que

$$
\mathbb{P}\left[\gamma_{\boldsymbol{\eta}, \boldsymbol{\eta}^{\prime}} \text { ser inútil em todo } \Gamma \in \Upsilon_{c N}\right] \leq \sum_{\substack{\gamma_{\boldsymbol{\eta}, \boldsymbol{\eta}^{\prime}}: \\\left|\gamma_{\boldsymbol{\eta}, \boldsymbol{\eta}^{\prime}}\right| \leq c N}} \mathbb{P}\left[\gamma_{\boldsymbol{\eta}, \boldsymbol{\eta}^{\prime}} \text { ser inútil }\right] \leq N^{c N} e^{-c V_{1}^{2}}
$$


Como o último termo da desigualdade acima é somável, a conclusão então segue do Primeiro Lema de Borel-Cantelli.

Como mencionamos anteriormente, qualquer que seja o conjunto de caminhos $\Gamma$ escolhido, o caminho $\gamma_{\overline{\boldsymbol{\sigma}}, \overline{\boldsymbol{\sigma}}^{\prime}} \in \Gamma$. Isto segue por causa da propriedade de completude de $\Gamma$. Pelo Lema 6.3.2, para todo $N$ grande o suficiente, com $\mathbb{P}$-probabilidade 1, existe ao menos um elo em $\gamma_{\overline{\boldsymbol{\sigma}}, \overline{\boldsymbol{\sigma}}^{\prime}}^{\text {int }}$ digamos $e_{0}=\left(\boldsymbol{\sigma}_{0}, \boldsymbol{\sigma}_{0}^{\prime}\right)$, tal que algum dos sítios $\boldsymbol{\sigma}_{0}$ ou $\boldsymbol{\sigma}_{0}^{\prime}$ é útil. Logo,

$$
\begin{aligned}
\mathrm{K}_{N}(\Gamma) & =\frac{V}{Z} \max _{e=\left(\boldsymbol{\sigma}, \boldsymbol{\sigma}^{\prime}\right)} e^{\beta\left[H(\boldsymbol{\sigma}) \vee H\left(\boldsymbol{\sigma}^{\prime}\right)\right]} \sum_{\gamma_{\boldsymbol{\eta}, \boldsymbol{\eta}^{\prime}} \ni e}\left|\gamma_{\boldsymbol{\eta}, \boldsymbol{\eta}^{\prime}}\right| e^{-\beta\left[H(\boldsymbol{\eta})+H\left(\boldsymbol{\eta}^{\prime}\right)\right]} \\
& \geq \frac{1}{Z} e^{\beta\left[H\left(\boldsymbol{\sigma}_{0}\right) \vee H\left(\boldsymbol{\sigma}_{0}^{\prime}\right)\right]} \sum_{\gamma_{\boldsymbol{\eta}, \boldsymbol{\eta}^{\prime} \ni e_{0}}} e^{-\beta\left[H(\boldsymbol{\eta})+H\left(\boldsymbol{\eta}^{\prime}\right)\right]} \\
& \geq \frac{1}{Z} e^{-\delta \beta N} e^{-\beta\left[H(\overline{\boldsymbol{\sigma}})+H\left(\overline{\boldsymbol{\sigma}}^{\prime}\right)\right]} .
\end{aligned}
$$

Aplicando agora o liminf com $N \uparrow \infty$ na desigualdade acima e invocando o Corolário 2.1.1 e o fato que $\overline{\boldsymbol{\sigma}}, \overline{\boldsymbol{\sigma}}^{\prime} \in \mathcal{T}$, então, com $\mathbb{P}$-probabilidade 1 ,

$$
\begin{aligned}
& \liminf _{N \uparrow \infty} \frac{1}{N} \log \mathrm{K}_{N}(\Gamma) \geq-\beta \limsup _{N \uparrow \infty} \frac{1}{N}\left(H(\overline{\boldsymbol{\sigma}})+H\left(\overline{\boldsymbol{\sigma}}^{\prime}\right)\right)-\limsup _{N \uparrow \infty} \frac{1}{N} \log Z-\delta \beta \\
& \geq 2 \beta \beta_{\star}(\sqrt{a p}-\sqrt{\bar{a} \bar{p}})-F_{2}(\beta)-\delta \beta \\
& \stackrel{(6.1 .8)}{=} 2 \beta\left(\beta_{1} a+\beta_{2} \bar{a}\right)-F_{2}(\beta)-\delta \beta,
\end{aligned}
$$

em que usamos também que $\frac{V_{j}}{N} \rightarrow p_{j}$ quando $N \uparrow \infty$ (cf. (2.1.14)).

Como a desigualdade acima vale para todo $\delta>0$ e estamos supondo $\beta \geq \beta_{2}$, de (2.1.56), obtemos que

$$
\liminf _{N \uparrow \infty} \frac{1}{\beta N} \log \mathrm{K}_{N}(\Gamma) \geq \beta_{1} a+\beta_{2} \bar{a}, \quad \mathbb{P}-\text { q.c. }
$$

e o Teorema 6.1.2 está demonstrado. 


\section{Apêndice A}

\section{Resultados de Matemática Geral}

Teorema A.0.1 (Teorema da Projeção sobre um Convexo Fechado - [Bre83]). Seja $H$ um espaço de Hilbert e seja $K \subset H$ um convexo fechado não vazio. Então, para cada $x \in \mathbb{R}^{n}$ existe um único $y \in K$ tal que

$$
\mathrm{d}(x, y)=\inf _{z \in S} \mathrm{~d}(x, z)=\mathrm{d}(x, S) .
$$

Além disso, y é caracterizado pela propriedade:

$$
y \in K \quad \text { e } \quad\langle z-y, x-y\rangle \leq 0, \quad \forall z \in K .
$$

Lema A.0.1 ([DD02]). Sejam $x, y \in \mathbb{R}^{n}$ tais que $x_{1} \geq x_{2} \geq \cdots \geq x_{n} \geq 0$ e $\lambda_{k} \stackrel{\text { def }}{=} \sum_{i=1}^{k} y_{i} \geq 0$ para todo $k=1, \ldots, n$. Então,

$$
\sum_{i=1}^{n} x_{i} y_{i} \geq 0
$$

Demonstração. Defina $\lambda_{0} \equiv 0$. Como $y_{i}=\lambda_{i}-\lambda_{i-1}$, para todo $i=1, \ldots, n$, segue que

$$
\begin{aligned}
\sum_{i=1}^{n} x_{i} y_{i}=\sum_{i=1}^{n} x_{i}\left(\lambda_{i}-\lambda_{i-1}\right) & =x_{1} \lambda_{1}+x_{2}\left(\lambda_{2}-\lambda_{1}\right)+\cdots+x_{n}\left(\lambda_{n}-\lambda_{n-1}\right) \\
& =x_{n} \lambda_{n}+\sum_{i=1}^{n-1}\left(x_{i}-x_{i+1}\right) \lambda_{i} \\
& \geq 0 .
\end{aligned}
$$

Proposição A.0.1 ([HUL96] - Seção 2.3 - pág. 111). Se $C$ é um conjunto compacto, então $\operatorname{ext} C \neq \varnothing$, onde ext $C$ é o conjunto de pontos extremos de $C$.

Proposição A.0.2 ([HUL96] - Seção 2.4 - pág. 115). Seja $C \subset \mathbb{R}^{n}$ um convexo e compacto. Para todo $s \in \mathbb{R}^{n}$, vale

$$
\max _{x \in C}\langle s, x\rangle=\max _{x \in \operatorname{ext} C}\langle s, x\rangle,
$$

onde extC é o conjunto de pontos extremos de $C$. 
Teorema A.0.2 ([R $\left.{ }^{+} 64\right]$ - pág. 89). Se $f$ é uma função contínua de um espaço métrico compacto $X$ em $\mathbb{R}^{k}$, então $f(X)$ é fechado e limitado. Portanto, $f$ é limitada.

Lema A.0.2. Sejam $a, b \in \mathbb{R} \operatorname{com} a<b$. Se $f, g:[a, b] \rightarrow \mathbb{R}$ são funções limitadas e $c>0$, então

$$
\max _{x \in[a, b]}\{-f(x)\}=-\min _{x \in[a, b]} f(x), \quad \max _{x \in[a, b]}\{c f(x)+g(x)\} \leq c \cdot \max _{x \in[a, b]} f(x)+\max _{x \in[a, b]} g(x)
$$

$\mathrm{e}$

$$
\min _{x \in[a, b]}\{c f(x)+g(x)\} \geq c \cdot \min _{x \in[a, b]} f(x)+\min _{x \in[a, b]} g(x) .
$$




\section{Apêndice B}

\section{Resultados de Teoria de Probabilidade}

Lema B.0.1 (Desigualdade de Chernoff). Sejam $X_{1}, X_{2}, \ldots, X_{k}$ variáveis aleatórias independentes, tais que $X_{i}=1$ com probabilidade $p_{i}$ e $X_{i}=0$ com probabilidade $1-p_{i}$. Seja $Y_{k}=\sum_{i=1}^{k} X_{i}$ e denotemos por $\mu=\mathbb{E}\left(Y_{k}\right)=\sum_{i=1}^{k} p_{i}$. Então,

$$
\mathbb{P}\left[Y_{k}>(1+\delta) \mu\right] \leq e^{-\frac{\delta^{2} \mu}{2+\delta}}, \quad \forall \delta>0
$$

Em particular, se $p_{i}=p$, para todo $i=1, \ldots, k$, e fazendo $\theta=1+\delta$, temos que

$$
\mathbb{P}\left[Y_{k}>\theta k p\right] \leq e^{-\frac{1}{3}(\theta-1) k p}, \quad \forall \theta \geq 2
$$

Lema B.0.2. Sejam $X_{1}, \ldots, X_{k}$ uma sequência de variáveis aleatórias contínuas i.i.d. sobre $(\mathscr{S}, \Sigma, \mathbb{P})$ e $Y_{1}, \ldots, Y_{k}$ suas estatísticas de ordem crescentes. Então,

$$
\mathbb{P}\left[Y_{k}>y \mid Y_{k-1}=x\right]=\frac{\mathbb{P}\left[X_{1}>y\right]}{\mathbb{P}\left[X_{1}>x\right]}, \quad \forall y>x
$$

em que $F_{X_{1}}$ é a função de distribuição acumulada de $X_{1}$.

Lema B.0.3 (Lema de Dominação - [FIKP98]). Sejam $X_{1}, \ldots, X_{k+1}$ uma sequência de variáveis aleatórias contínuas i.i.d. sobre $(\mathscr{I}, \Sigma, \mathbb{P})$ com função de distribuição acumulada $F$, $Y_{k}=\max \left\{X_{1}, \ldots, X_{k+1}\right\}$ e $M \in\{1, \ldots, k+1\}$ tal que $Y_{k}=X_{M}$. Para $k=1, \ldots, m$, defina

$$
Y_{m}= \begin{cases}X_{m}, & \text { se } m<M ; \\ X_{m+1}, & \text { c.c. }\end{cases}
$$

Então,

$$
\mathbb{P}\left[Y_{1}<t_{1}, \ldots, Y_{k}<t_{k}\right] \geq \prod_{m=1}^{k} \mathbb{P}\left[X_{1}<t_{m}\right] .
$$

Lema B.0.4 ([FIKP98] - pág. 925). Sejam $X_{1}, \ldots, X_{k}$ uma sequência de variáveis aleatórias i.i.d. gaussianas padrão sobre $(\mathscr{S}, \Sigma, \mathbb{P})$ e seja $m_{k}=\min \left\{X_{1}, \ldots, X_{k}\right\}$. Então, para todo $\varepsilon>0$, com $\mathbb{P}$-probabilidade 1 para todo mas um número finito de índices $k$,

$$
m_{k} \geq-\sqrt{2(1+\varepsilon) \log k}
$$


APÊNDICE B 


\section{Bibliografia}

[ABG01] G. B. Arous, A. Bovier, and V. Gayrard. Glauber dynamics of the random energy model i. metastable motion on the extreme states. 2001. 3

[ABG02] G. B. Arous, A. Bovier, and V. Gayrard. Aging in the random energy model. Physical review letters, 88(8):087201, 2002. 3

[ABG03a] G. B. Arous, A. Bovier, and V. Gayrard. Glauber dynamics of the random energy model. Communications in mathematical physics, 236(1):1-54, 2003. 3

[ABG03b] G. B. Arous, A. Bovier, and V. Gayrard. Glauber dynamics of the random energy model: Ii. aging below the critical temperature. Communications in mathematical physics, 236(1), 2003. 3

[BCKM98] J-P. Bouchaud, L. F. Cugliandolo, J. Kurchan, and M. Mezard. Out of equilibrium dynamics in spin-glasses and other glassy systems. Spin glasses and random fields, pages 161-223, 1998. 2

[BK04a] A. Bovier and I. Kurkova. Derrida's generalised random energy models 1: models with finitely many hierarchies. 40(4):439-480, 2004. 3, 40, 66, 71, 74, 79

[BK04b] A. Bovier and I. Kurkova. Derrida's generalized random energy models 2: models with continuous hierarchies. 40(4):481-495, 2004. 3

[Bre83] H. Brezis. Analyse fonctionnelle. 1983. 83

[CCP87] D. Capocaccia, M. Cassandro, and P. Picco. On the existence of thermodynamics for the generalized random energy model. Journal of statistical physics, 46(3-4):493-505, 1987. $3,11,12,13,22,35,38,41,50$

[ČW17] J. Černỳ and T. Wassmer. Aging of the metropolis dynamics on the random energy model. Probability Theory and Related Fields, 167(1-2):253-303, 2017. 3 
[DD02] T. C. Dorlas and W. M. B. Dukes. Large deviation approach to the generalized random energy model. Journal of Physics A: Mathematical and General, 35(20):4385, 2002. $3,13,35,61,83$

[Der80] B. Derrida. Random-energy model: Limit of a family of disordered models. Physical Review Letters, 45(2):79, 1980. 1, 6

[Der85] B. Derrida. A generalization of the random energy model which includes correlations between energies. Journal de Physique Lettres, 46(9):401-407, 1985. 2, 5, 6, 7, 11

[DS91] P. Diaconis and D. Stroock. Geometric bounds for eigenvalues of markov chains. The Annals of Applied Probability, pages 36-61, 1991. 4, 16, 17, 19, 20, 21

[FG18] L. R. Fontes and V. Gayrard. Asymptotic behavior and aging of a low temperature cascading 2-grem dynamics at extreme time scales. arXiv preprint arXiv:1801.08832, 2018. 75

[FIKP98] L. R. G. Fontes, M. Isopi, Y. Kohayakawa, and P. Picco. The spectral gap of the rem under metropolis dynamics. Annals of Applied Probability, pages 917-943, 1998. $3,16,17,21,22,35,42,56,73,76,77,78,85$

[Gay92] V. Gayrard. Thermodynamic limit of theq-state potts-hopfield model with infinitely many patterns. Journal of Statistical Physics, 68(5):977-1011, Sep 1992. 80

[Gay16a] V. Gayrard. Aging in metropolis dynamics of the rem: a proof. arXiv preprint arXiv:1602.06081, 2016. 3

[Gay16b] V. Gayrard. Convergence of clock processes and aging in metropolis dynamics of a truncated rem. In Annales Henri Poincaré, volume 17, 2016. 3

[HHJ90] R. A Horn, R. A Horn, and C. R. Johnson. Matrix analysis. Cambridge university press, 1990. 16

[HUL96] J-B. Hiriart-Urruty and C. Lemarechal. Convex Analysis and Minimization Algorithms I: Fundamentals, volume 305. Springer Science \& Business Media, 1996. 83

[JR06] N. K. Jana and B. V. Rao. Generalized random energy model. Journal of statistical physics, 123(5):1033-1058, 2006. 3, 5, 6, 8, 9, 13

[Mat00] P. Mathieu. Convergence to equilibrium for spin glasses. Communications in Mathematical Physics, 215(1):57-68, 2000. 3 
[MP] P. Mathieu and P. Picco. Convergence to equilibrium for finite markov processes with application to the random energy model.(2000) http://front. math. ucdavis. edu/math. PR/0307148. 3

[OP84] E. Olivieri and P. Picco. On the existence of thermodynamics for the random energy model. Communications in Mathematical Physics, 96(1):125-144, 1984. 11, 35, 41

[Pic92] P. Picco. On the REM and the GREM. Springer, 1992. 50

$\left[\mathrm{R}^{+} 64\right] \mathrm{W}$. Rudin et al. Principles of mathematical analysis, volume 3. McGraw-hill New York, 1964. 84

[SC97] L. Saloff-Coste. Lectures on finite Markov chains. Springer Berlin Heidelberg, Berlin, Heidelberg, 1997. 19

[Sin91] A. Sinclair. Improved bounds for mixing rates of markov chains on combinatorial structures. Technical report, Technical report, Department of Computer Science, University of Edinburgh, 1991. 4, 19, 20, 21

[SJ89] A. Sinclair and M. Jerrum. Approximate counting, uniform generation and rapidly mixing markov chains. Information and Computation, 82(1):93-133, 1989. 20

[SK75] D. Sherrington and S. Kirkpatrick. Solvable model of a spin-glass. Physical review letters, 35(26):1792, 1975. 1 